\begin{tabular}{|c|l|}
\hline Title & Mean curvature flow through singul arities for surfaces of rotation \\
\hline Author(s) & A Itschuler, Steven; A ngenent, Sigurd; Giga, Y oshikazu \\
\hline Citation & Hokkaido University Preprint Series in Mathematics, 130, 1-62 \\
\hline Issue Date & 1991-12 \\
\hline DOI & 10.14943/83275 \\
\hline Doc URL & http://hdl.handle.net/2115/68877 \\
\hline Type & bulletin (article) \\
\hline File Information & pre130.pdf \\
\hline
\end{tabular}

Instructions for use 


\section{Mean Curvature Flow through Singularities for Surfaces of Rotation}

S. Altschuler, S. Angenent and Y. Giga

Series $\sharp 130$. December 1991 


\section{HOKKAIDO UNIVERSITY}

\section{PREPRINT SERIES IN MATHEMATICS}

104: H. Takamura, Global existence of classical solutions to nonlinear wave equations with spherical symmetry for small data with noncompact support in three space dimensions, 14 pages. 1991.

\#105: R. Agemi, Blow-up of solutions to nonlinear wave equations in two space dimensions, 11 pages. 1991.

\#106: T. Nakazi, Extremal problems in $H^{p}, 13$ pages. 1991.

\#107: T. Nakazi, $\rho$-dilations and hypo-Dirichlet algebras, 15 pages. 1991.

108: A. Arai, An abstract sum formula and its applications to special functions, 25 pages. 1991.

109: Y.-G. Chen, Y. Giga and S. Goto, Analysis toward snow crystal growth, 18 pages. 1991.

$\sharp 110$ : T. Hibi, M. Wakayama, A q-analogue of Capelli's identity for $G L(2), 7$ pages. 1991.

$\sharp 111$ : T. Nishimori, A qualitative theory of similarity pseudogroups and an analogy of Sacksteder's theorem, 13 pages. 1991.

112: K. Matsuda, An analogy of the theorem of Hector and Duminy, 10 pages. 1991.

\#113: S. Takahashi, On a regularity criterion uo to the boundary for weak solutions of the Navier-Stokes equations, 23 pages. 1991.

114: T. Nakazi, Sum of two inner functions and exposed points in $H^{1}, 18$ pages. 1991.

\#115: A. Arai, De Rham operators, Laplacians, and Dirac operators on topological vector spaces, 27 pages. 1991.

\#116: T. Nishimori, A note on the classification of non-singular flows with transverse similarity structures, 17 pages. 1991.

117: T. Hibi, A lower bound theorem for Ehrhart polynomials of convex polytopes, 6 pages. 1991.

118: R. Agemi, H. Takamura, The lifespan of classical solutions to nonlinear wave equations in two space dimensions, 30 pages. 1991.

\#119: S. Altschuler, S. Angenent and Y. Giga, Generalized motion by mean curvature for surfaces of rotation, 15 pages. 1991.

\#120: T. Nakazi, Invariant subspaces in the bidisc and commutators, 20 pages. 1991.

\#121: A. Arai, Commutation properties of the partial isometries associated with anticommuting self-adjoint operators, 25 pages. 1991.

122: Y.-G. Chen, Blow-up solutions to a finite difference analogue of $u_{t}=\Delta u+u^{1+\alpha}$ in $N$-dimensional balls, 31 pages. 1991.

\#123: A. Arai, Fock-space representations of the relativistic supersymmetry algebra in the two-dimensional spacetime, 13 pages. 1991.

$\sharp 124: \quad$ S. Izumiya, The theory of Legendrian unfoldings and first order differential equations, 16 pages. 1991.

$\sharp 125$ : T. Hibi, Face number inequalities for matroid complexes and Cohen-Macaulay types of Stanley-Reisner rings of distributive lattices, 17 pages. 1991.

\#126: S. Izumiya, Completely integrable holonomic systems of first order differential equations, 35 pages. 1991.

127: G. Ishikawa, S. Izumiya and K. Watanabe, Vector fields near a generic submanifold, 9 pages. 1991.

\#128: A. Arai, I. Mitoma, Comparison and nuclearity of spaces of differential forms on topological vector spaces, 27 pages. 1991.

\#129: K. Kubota, Existence of a global solution to a semi-linear wave equation with initial data of non-compact support in low space dimensions, 53 pages. 1991. 
Mean Curvature Flow through Singularities for

Surfaces of Rotation.

Steven Altschuler, Sigurd Angenent and Yoshikazu Giga. 


\section{Table of contents.}

§1. Introduction. . . . . . . . . . . . . . . . . . . . . 1

Background to the problem

Main results

Brief outline of the paper

Acknowledgements

§2. Notation and Smooth Solutions . . . . . . . . . . . . . 7

Sign and orientation conventions

$\alpha$-Domains

Graph equations

The Evans-Spruck estimates

§3. The Inner and Outer Evolution. . . . . . . . . . . . . . . . . . 11

Definition of Inner and Outer Evolution

3.2. Comparison Lemma

Convergence and Separation Properties

3.3. Approximation lemma

3.4. Monotone Convergence Theorem

3.5. Separation theorem

3.6. Continuity in time

Regular Evolutions, or The Interior of $\Gamma(t)$

3.9. Regularity lemma for Monotone Motion

3.11. Second regularity lemma

§4. Intersections and the Sturmian theorem. . . . . . . . . . . . . 21

Sturm's classical result

4.1. Theorem on Intersections

4.2. Attracting Axis Theorem

The Catenoid and its generalizations

4.4. Moving Caps Lemma

Proof of Theorem 4.3.a

Proof of Theorem 4.3.b

Proof of Theorem 4.2

4.6. Corollary

The necks of an evolving domain

$\S 5$. Formation of a singularity.

A final segment of a smooth evolution

5.3. Limit Surface Lemma

Domains without necks

A necessary condition for formation of a singularity

Asymptotics of a shrinking neck

5.7. Contraction Rate Theorem

Proof of the Contraction Rate Theorem

5.9. Cylindrical Blow-up Theorem

5.10. Corollary (Ilmanen)

Proof of the blow-up theorem

The inner evolution at time $t=T$

§6. After the singularity. . . . . . . . . . . . . . . . . . 47

Instant smoothness

The complete inner evolution

\$7. Inner and Outer Evolutions coincide. . . . . . . . . . . . . . . 51

Short time regularity for $\alpha$-domains

$\S 8$. Hamilton's incredibly shrinking dumbbell. . . . . . . . . . . 56 
Table of Figures.

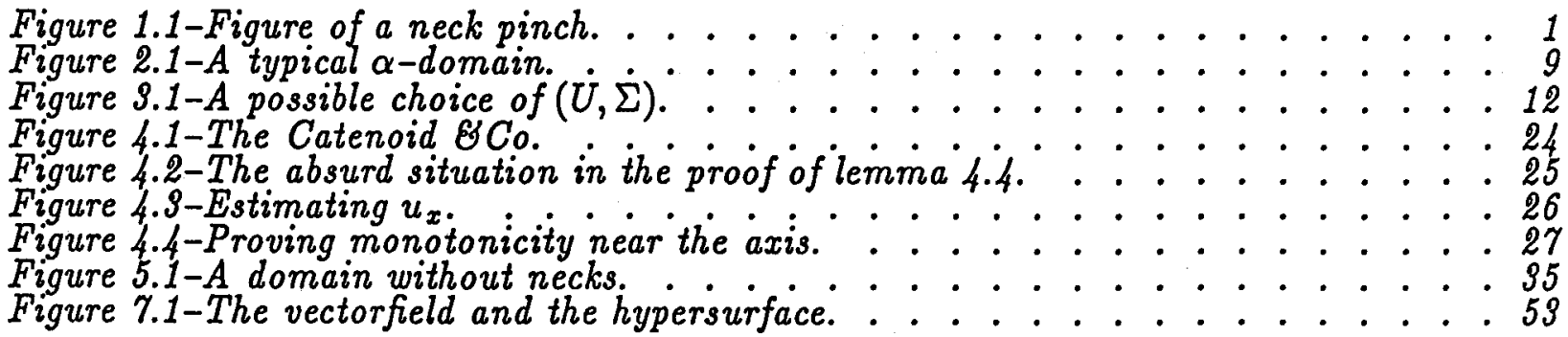




\title{
Mean Curvature Flow through Singularities for Surfaces of Rotation.
}

\author{
S. Altschuler, S. B. Angenent and Y. Giga
}

\section{$\S 1$. Introduction.}

A smooth one-parameter family of hypersurfaces $\Gamma_{t} \subset \mathrm{R}^{n+1}(0<t<T)$ evolves by its mean curvature if its normal velocity at each point $p \in \Gamma_{t}$ coincides with the mean curvature of $\Gamma_{t}$ at $p$. In this paper, we consider the generalized "viscosity" solutions of mean curvature evolution which were introduced by Chen, Giga \& Goto [CGG1] as well as by Evans \& Spruck [ES1]. We shall be mainly interested in those solutions whose initial value is a compact, smooth and rotationally symmetric hypersurface $\Gamma_{0} \subset R^{n+1}$ which is given as the graph of a function $r=u(x)$, in cylindrical coordinates. The solutions of the mean curvature flow provided by [CGG1, ES1] need not be smooth hypersurfaces-indeed, in some cases they may even have a nonempty interior. Our main result states that such a generalized solution is a smooth hypersurface, except at an isolated set of points in spacetime $[0, \infty) \times R^{n+1}$.

Background to the problem. Concerning smooth solutions of mean curvature flow, Huisken [H1] showed that any solution which starts out as a smooth, compact and convex hypersurface remains so until it shrinks to a "round point"; its asymptotic shape just before it disappears is a sphere. He proved this result for hypersurfaces of $R^{n+1}$ with $n \geq 2$, but Gage and Hamilton [GH] showed that it still holds when $n=1$, the case of curves in the plane. The methods used in [H1, GH] do not resemble each other. Gage and Hamilton also observed that any smooth family of plane immersed curves which moves by its mean curvature and which starts out as an embedded curve remains embedded. Grayson [Gr1] proved the remarkable fact that such a family must become convex before it becomes singular. Thus, any embedded curve in the plane will shrink to a "round point" under mean curvature flow. It is natural to ask if the same is true in higher dimensions. While it is true that smooth solutions remain embedded if their initial surface is embedded, Grayson [Gr2] also showed that there exist smooth solutions which become singular before they shrink to a point. His example consisted of a barbell: two spherical surfaces connected by a sufficiently thin "neck." In this example the inward curvature of the neck is so large that it will force the neck to pinch before the two spherical ends can shrink appreciably. 
See figure 1.1.

Figure 1.1-Figure of a neck pinch.

One's geometric or physical intuition (imagine the surface to be the boundary of a piece of ice and that mean curvature flow models the melting of the ice) suggests that even though the smooth solution in Grayson's example cannot be extended as a smooth solution, some sort of generalized solution might exist. In this generalized solution one would expect the two halves of the barbell to separate, and afterwards evolve independently of each other; one would also expect them to become smooth surfaces immediately after separation.

A similar situation occurs in the evolution by curvature for plane immersed curves. Here little loops can attract curvature and force smooth solutions to become singular and form cusp-like singularities before they can shrink to a point; and here too, it is very plausible that a generalized solution can be constructed.

The second author [A3] gave an ad hoc construction of a generalized solution for the motion by curvature of immersed curves. He analyzed the behaviour of a smooth solution as it becomes singular, and showed that a singular limit curve exists at the time of a singularity. Next, he showed that this singular limit curve, after removing some "spurious hairs," is nice enough to be the initial data for another smooth solution of mean curvature flow. By repeating these two steps he obtained a solution with isolated singularities; it turned out that such singularities occur at most a finite number of times, so that the solution one gets in this way is maximal. Although this method provides a lot of detailed information about the constructed solution, it has a few disadvantages: The "spurious hairs" which have to be removed are difficult to analyze, and it is not even clear if they really occur; and, apart from the construction itself, the method does not provide a simple definition of what a solution is.

Following an idea of Calabi, the first author and Grayson gave another construction of a generalized solution. The idea in this construction is to regularize the equation. This regularization is achieved by adding an extra (say vertical) dimension to the plane, and by replacing the curve by a covering curve which slowly spirals upwards, say with slope $\varepsilon>0$. The spiraling curve is then a graph over the vertical axis, and one can construct a smooth solution of the mean curvature flow with this spiraling curve as initial data. The fact that this curve is a graph can be used to show that it remains a graph over the vertical axis, and that it exists forever-in fact it converges to a vertical line as $t \rightarrow \infty$ without ever becoming singular. Using the upward slope $\varepsilon$ as a small parameter one then gets a one parameter family of evolving space curves. In [AG] it is shown that this family converges as $\varepsilon$ tends to zero; the limit is the desired generalized solution of the planar motion by curvature. While this construction avoids the problem of "spurious hairs," and also has 


\section{Mean Curvature Flow Through Singularities for Surfaces of Rotation.}

the attractive feature that the entire generalized solution is constructed in one step, it is not clear how one could generalize it to the higher dimensional mean curvature motions.

Yet another definition of weak solutions to mean curvature flow was given much earlier by Brakke in his seminal work [B]. He shows how, using geometric measure theory, one can construct a theory of generalized solutions for varifolds in $\mathrm{R}^{n+1}$ of arbitrary dimension and co-dimension. He proves the existence of at least one global solution for any initial data; in fact, he shows in an example that one initial datum may have many different solutions. This feature of his theory is related to the "fattening" phenomenon of the viscosity solutions.

The generalized solutions with which we shall be concerned in this paper were introduced independently by Chen, Goto and the third author [CGG1], as well as by Evans and Spruck [ES1]. The idea in both approaches is to regard a hypersurface as the zeroset of a function $\psi:[0, \infty) \times \mathbf{R}^{n+1} \rightarrow \mathbf{R}$, and to derive an equation which guarantees that its zeroset will evolve by its mean curvature. Many equations will have this property, but if one requires that not only the zeroset, but all levelsets of the function $\psi$ evolve by their mean curvature, then one finds that $\psi$ must satisfy the following PDE:

$$
\psi_{t}-|\nabla \psi| \operatorname{div}\left(\frac{\nabla \psi}{|\nabla \psi|}\right)=0
$$

We shall refer to this equation as the level set equation. Its derivation assumes that $\psi$ is at least $C^{2}$, and it is only valid for those levelsets of $\psi$ which are regular, i.e. on which $\nabla \psi$ does not vanish.

The idea to represent hypersurfaces as levelsets is of course common in differential geometry; in the present context it goes back to Ohta, Jasnow and Kawasakj [OJK], who used the levelset equation to derive a scaling law for "dynamic structure functions" from a physical point of view; the idea to use the levelset equation to study motion by mean curvature numerically was used by Sethian, and later by Sethian \& Osher [Se1, OS, Se2].

From the analyst's point of view the level set equation is not very nice. It is a parabolic equation, but it is very degenerate, so that classical techniques and results cannot be expected to apply. Nevertheless, it was shown in [CGG1, ES1] that the theory of viscosity solutions for nonlinear elliptic and parabolic PDE, as presented in [CIL] can be adapted so that it will apply to the level set equation. In fact, in [CGG1, CGG2, GG1] a larger class of equations was considered.

The main result of [CGG1, ES1] states that the level set equation has a unique continuous "viscosity" solution $\psi:[0, \infty) \times \mathbf{R}^{n+1} \rightarrow \mathrm{R}$ for any given continuous initial data $\psi(x, 0)=\psi_{0}(x)$ for which $\psi_{0}-a$ has compact support, for some $a \in \mathbf{R}$. The most technical step in the proofs of both [CGG1] and [ES1] was to establish a comparison principle for viscosity sub- and supersolutions. The proof of the key comparison principle in [CGG1] has been simplified in [GGIS].

Using their solution to the level set equation, the authors of [CGG1, ES1] defined generalized solutions of the mean curvature flow as follows. Let $\Gamma_{0} \subset R^{n+1}$ be a compact hypersurface, and choose some function $\psi_{0} \in C^{0}\left(R^{n+1}\right)$ with $\Gamma_{0}$ as zeroset, and with $\psi_{0}-a$ 


\section{Altschuler, Angenent and Giga}

compactly supported for some $a<0$; then let $\psi(x, t)$ be the unique corresponding viscosity solution of the levelset equation, and put $\Gamma(t)=\left\{x \in \mathrm{R}^{n+1}: \psi(x, t)=0\right\}$. Of course one has to verify that the choice of $\psi_{0}$ does not affect the zeroset of the solution $\psi$, and this is done in [CGG1, ES1].

To define the evolution one does not have to assume that $\Gamma_{0}$ is smooth. In fact, the definition can be applied to any compact set $\Gamma_{0} \subset \mathrm{R}^{n+1}$. Conversely, the only "regularity" of the solution $\Gamma(t)$ which the definition provides is that $\Gamma(t)$ is a compact subset of $\mathbf{R}^{n+1}$. There have been some partial regularity results which go beyond this (see [ES2, ES3, GG2, I1, I2, ESS, CGG2]), but the general question of regularity is still far from understood.

A particularly disturbing aspect of the solutions of [CGG1, ES1] is that for $t>0$ a solution $\Gamma(t)$ may have a nonempty interior, even if the initial hypersurface is smooth, except for a few isolated singularities. An example is given in [ES1], where it is argued that the solution in $\mathbf{R}^{2}$ whose initial position is a "figure eight" (or any other smooth curve with one double point), has nonempty interior.

Intuitively one can understand this fattening of solutions as follows: If $\Gamma_{0}$ is a possibly singular hypersurface, then one can try to construct a generalized solution to mean curvature flow by first approximating $\Gamma_{0}$ with smooth hypersurfaces $\Gamma_{i} \rightarrow \Gamma_{0}$, and then taking the limit of the corresponding solutions $\Gamma_{i}(t) \rightarrow \Gamma_{0}(t)$. In particular, one can approximate $\Gamma_{0}$ from the inside and obtain one solution, and one can approximate $\Gamma_{0}$ from the outside to obtain another solution. If these "inner" and "outer" solutions coincide then it follows from the maximum principle that any approximating sequence will have the same limiting solution. If they are different, then there is no preferred (smooth) solution, and the generalized solution will consist of the entire region between the inner and outer solutions.

One reason why it is of interest to know when a generalized solution will "fatten" is the relation with semilinear heat equations. Recently, De Giorgi [DG] proposed another weak formulation of solutions of mean curvature flow based on a singular limit of a reactiondiffusion equation of the type

$$
u_{t}=\Delta u+\frac{1}{\varepsilon^{2}} u\left(1-u^{2}\right) .
$$

It is not known if his solution agrees with ours, although our inner solution is obtained by Evans, Soner \& Souganidis [ESS] as a singular limit of solutions of (1.2). They consider the solution $u^{\varepsilon}(x, t)$ with initial condition $u^{\varepsilon}(x, 0)=2 \chi_{D_{0}}(x)-1$; here $\chi_{D_{0}}$ is the characteristic function of the region $D_{0}$ enclosed by some compact hypersurface $\Gamma_{0}$. Assuming that the generalized solution $\Gamma(t)$ corresponding to $\Gamma_{0}$ does not "fatten," it is shown in [ESS] that $u^{\varepsilon}(x, t)$ converges to $2 \chi_{D(t)}-1$, where $D(t)$ is region enclosed by $\Gamma(t)$ at time $t$.

There is no known necessary and sufficient condition which describes when a solution will not "fatten." But there are a few sufficient conditions, the simplest of which requires the initial hypersurface to be smooth with strictly positive mean curvature (w. r. t. its inward normal-see theorem 3.10); a generalization of this criterion was given by Soner in [So, SS]. Soner [So] has used the results of [CGG1] to set up another theory of generalized solutions of mean curvature flow. Unlike the theories of [ES1, CGG1], but more like 


\section{Mean Curvature Flow Through Singularities for Surfaces of Rotation.}

Brakke's theory [Br], Soner's solutions are again not uniquely determined by their initial data, but they always lie in between the inner and outer evolutions, in our terminology.

After this work was completed, we were informed of recent work of H. M. Soner and P. E. Souganidis [SS] closely related to $\S 5$ and $\S 7$. They proved the cylindrical blow-up theorem 5.9 for initial data under a symmetry condition which forces the neck to have no motion along the $x$-axis. They also prove regularity of the inner evolution for surfaces given by rotating a circle around the $x$-axis.

Main results. We have studied the generalized evolution corresponding to a smooth compact hypersurface $\Gamma_{0} \subset R^{n+1}$ which is obtained by rotating the graph of a function $r=u(x), a<x<b$ around the $x$-axis. Denote this solution by $\Gamma(t)$.

1.1. Theorem. Outside of the $x$-axis the solution $\Gamma(t)$ is a smooth family of smooth hy. persurfaces evolving by their mean curvature. In particular, the solution does not "fatten," $\Gamma(t)$ always has empty interior.

There is a finite sequence of times $0=t_{0}<t_{1}<\cdots<t_{l}$ such that the hypersurfaces $\Gamma(t)$ are smooth and compact in all of $\mathrm{R}^{n+1}$, including the $x$-axis, when $t_{j-1}<t<t_{j}$, $j=1,2, \ldots, l$. The solution is empty for $t>t_{l}$.

The time $t_{l}$ is called the extinction time of the solution. The solution $\Gamma(t)$ is not necessarily connected, even though the initial hypersurface $\Gamma_{0}$ is. However, the number of components of $\Gamma(t)$ can only change at the singular times $t_{j}$. The next theorem says how.

1.2. Theorem. Let $\Gamma_{*}(t)$ be a component of $\Gamma(t)$ which becomes singular at $t_{j}$. Then we have the following alternative

(i) $\Gamma_{*}(t)$ shrinks to a point on the $x$-axis;

(ii) One or more "necks" of $\Gamma_{*}(t)$ have been pinched.

The number of necks $m_{*}(t)$ of any of the surfaces $\Gamma_{*}(t)$ is a nonincreasing function of $t \in\left(t_{j-1}, t_{j}\right)$. The hypersurface $\Gamma_{*}\left(t_{j}\right)$ is smooth away from the $x$-axis and its number of singular points on the $x$-axis is not more than $m_{*}(t-\varepsilon)$ for any $\varepsilon>0$.

Here a "neck" of $\Gamma(t)$ is a positive local minimum of the function $r=u(t, x)$ which describes $\Gamma(t)$ in cylindrical coordinates. By the Sturmian theorem (see section 3 ) the number of necks of the solution does not increase with time. Theorem 1.2 implies that at each singular time either a neck or a local maximum of $r=u(t, x)$ disappears, so that we have an estimate for the number of times which the solution can become singular.

1.3. Corollary. The number $l$ of singular times is not more than the number of positive local maxima and minima of the function $r=u(x)$ which defines the initial hypersurface.

For example, the barbell of figure 1.1 has one neck and two local maxima, so the corresponding generalized solution will be singular at most at three times. It may happen that the neck is not very thin, and that the solution becomes convex. By Huisken's first result the solution will then shrink to just one point. On the other hand, if the neck is thin enough then it will pinch, and the remaining two parts will each shrink to some point. In 
this case $l=2$ or $l=3$, depending on whether the two remaining parts disappear at the same time or not.

An intermediate case is also possible: the surface can shrink to a point, but never lose its neck. In section 8 we show, in a concrete example, that such a solution actually exists. Its existence was suggested by Hamilton, who invented this solution as an example of "type-II" blow-up; i.e. as an example of a solution whose maximal curvature blows up faster than $c\left(t_{1}-t\right)^{-1 / 2}$ for any $c>0$ (see [Alt]). We also show that this solution really exhibits type-II blow-up.

The example is given in dimensions 3 and higher, but the arguments we use in $\S 8$ may also be applied to the evolution of curves in the plane. They then provide a rigorous proof for the existence of non-regular ("fattening") solutions starting from a singular curve.

This first example of section 8 is symmetric w. $r$. t. reflections in the $x=0$ plane. We show that for non-symmetric solutions there is a similar intermediate way of becoming singular: a neck can pinch, but at the same time the part of the solution on one side of the neck shrinks to a point on the $x$-axis. In this situation the generalized solution remains connected after the singularity occurs.

Brief outline of the paper. We have organized the paper as follows.

Section 2. We introduce some notation, and recall the parabolic PDE's which describe the evolution of a surface of rotation. We also recall a Korevaar type gradient-estimate of Evans \& Spruck and also Ecker \& Huisken.

Section 3. We elaborate on the generalized solutions of [CGG1, ES1]. Because of the "fattening" phenomenon, and also for other reasons we have found it more natural to consider the inner and outer evolutions $D=\{(x, t): \psi>0\}$ and $E=\{(x, t): \psi \geq 0\}$ rather than the generalized solution $\Gamma=E \backslash D$ itself. It turns out that $D$ and $E$ are also uniquely determined by their initial values $D(0)=\{x: \psi(x, 0)>0\}, E(0)=\{x: \psi(x, 0) \geq 0\}$. The question whether $\Gamma$ fattens up or not, can now be reformulated as "is $E$ the closure of $D$ ?"

We state some theorems on the continuous dependence of the solution on initial data and on time, and also separation properties analogous to the maximum \& comparison principles for parabolic PDE's. These theorems turn out to have a more natural formulation in terms of inner and outer evolutions.

The final lemma in this section is a technical condition that prevents a solution from fattening; it will be used in section 7, where we apply it to rotationally symmetric solutions.

Section 4. In this section the Sturmian theorem is recalled and applied in the same way as it has been applied in [A1, A2, A3]. The idea of counting the intersections of graphs of solutions to scalar one-dimensional parabolic PDE was also used in the U.S.S.R. by Victor Galaktionov during the last 5 years: we refer to [GP] and the references given therein.

The two main results we get out of the Sturmian theorem are an a priori gradient estimate (theorem 4.3.b) and the attracting axis theorem. The latter says that once a neck gets very thin it must move monotonically towards the axis.

Section 5. Here we consider a smooth solution, and give a fairly detailed description of what happens to it as it becomes singular (the singular time is denoted by $T$.) We first 


\section{Mean Curvature Flow Through Singularities for Surfaces of Rotation.}

show that necks collapse at isolated points on the $x$-axis (a stronger version of this result was announced by X. Y. Chen). Then, we show that the surface converges uniformly to a limit surface at time $T$. Next, we find a blow-up rate for necks, based on the work of Huisken [H2], and prove that appropriately rescaled solutions converge to cylinders. A corollary of this work, pointed out to us by T. Ilmanen, is that necks are "flat." Next we show that the surface converges uniformly to a limit surface

Section 6 . In this section we show that the boundary of the inner weak solution $\partial D(t)$ satisfies theorem 1.1. The main step towards this statement consists of showing that the inner evolution immediately becomes smooth after each singular time $D\left(t_{i}\right)$. To show this we approximate $D\left(t_{i}\right)$ monotonically from the inside by smooth domains $D_{j}(0)(j=1,2, \cdots)$. By comparing the solutions $D_{j}(t)$ with shrinking cylinders and counting intersections we then show that the $D_{j}(t)$ stay smooth and are represented by "vertical graphs" $x=v_{j}(r, t)$, at least for a short time $(0, \delta)$ and near the intersection with the axis.

The Korevaar-Evans-Spruck estimates then allow us to show that $D(t)$ is a smooth family of smoothly bounded domains for $t_{i}<t<t_{i}+\delta$. Smoothness of $\partial D(t)$ away from the $x$-axis turns out to be easier to prove, using the interior estimate of theorem 4.3.b.

Section 7. The proof of theorem 1.1 is completed by showing that $E$ is the closure of $D$, i.e. that the solution $\Gamma$ does not fatten. The proof consists of an application of the "second regularity lemma" of section 3 . But to verify the hypotheses of this lemma we have to show that the outer evolution $E$ also splits up into components after any neck pinch. We do this by comparing it with a special similarity solution, the "shrinking doughnut," and this in turn is only possible because we have shown in section 5 that a pinched neck is "flat."

Section 8. This section is devoted to Hamilton's example of a special solution which shrinks to a point, but does not become convex.

The results in this paper were first announced in [AAG].

Acknowledgements. This work was started in the fall of 1990 when the first author was a postdoctoral fellow and the second and third authors were visiting at the IMA in Minneapolis. They would like to thank the IMA for providing an excellent environment in which to do research. This research was supported in part by the Institute for Mathematics and its Applications with funds provided by the National Science Foundation and the Air Force Office of Scientific Research AFOSR-89-0165.

The second author has been supported by a Sloan fellowship and an NSF-PYI grant; he is also grateful for the hospitality of the Ruhr Universität at Bochum, and the University of Leiden where he prepared parts of this paper in the summer of 1991.

\section{§. Notation and Smooth Solutions}

Troughout this paper we assume that $n \geq 2$; points in $\mathrm{R}^{n+1}, n \geq 1$ will be written as $(x, y)$ where $x \in \mathrm{R}$ and $y \in \mathrm{R}^{n}$; if $r$ and $y$ occur in the same context, then $r$ will always denote 


\section{Altschuler, Angenent and Giga}

$|y|$, i.e.

$$
r=\sqrt{y_{1}^{2}+\cdots+y_{n}^{2}} .
$$

The solid, open cylinder with radius $\rho$ will be denoted by $C_{\rho}$

$$
C_{\rho}=\{(x, y): r<\rho\}
$$

and its closure will be denoted by $\bar{C}_{\rho}$.

Sign and orientation conventions. If $\Gamma \subset \mathrm{R}^{n+1}$ is a smooth compact and connected hypersurface, then the Jordan-Brouwer separation theorem states that $R^{n+1} \backslash \Gamma$ has exactly two components. One of these is bounded, and will be refered to as the "inside" of $\Gamma$. The hypersurface $\Gamma$ is orientable, and admits precisely two smooth unit normal vector fields $\mathrm{n}: \Gamma \rightarrow S^{n}$. In this paper we shall always let $\mathrm{n}$ be the inward normal.

The second fundamental form at a point $P \in \Gamma$ is defined to be the bilinear form

$$
\mathbf{A}(\xi, \eta)=-\langle\xi, \nabla \mathbf{n}(P) \cdot \eta\rangle
$$

the principal curvatures of $\Gamma$ at $P$ are the eigenvalues $\kappa_{1}(P), \ldots, \kappa_{n}(P)$ of the so-called Weingarten map $-\nabla \mathbf{n}(P)$; by the mean curvature we mean $H=\kappa_{1}+\cdots+\kappa_{n}$ (and not $\left(\kappa_{1}+\cdots+\kappa_{n}\right) / n$, as many authors have done since the times of Gauß).

With these conventions the principal curvatures of the unit sphere in $\mathrm{R}^{n+1}$ are +1 , and, in general, the principal curvatures of any convex hypersurface are nonnegative.

$\alpha$-Domains. A surface of rotation can be decomposed into two parts, one close to the axis of symmetry, which may be represented as the union of two graphs $x=v_{1}(r), x=$ $v_{2}(r)$, and the complement which lies "away from the axis," and may be represented as the graph of a function $r=u(x)$. Throughout this paper we shall find it convenient to quantify the two phrases "close to the axis" and "away from the axis." Hence the following.

Let $\alpha>0$ be given and let $U \subset \mathrm{R}^{n+1}$ be an open set of the form

$$
U=\left\{(x, y) \in \mathbf{R}^{n+1}: r<u(x)\right\}
$$

for some $0 \leq u \in C(\mathrm{R})$.

2.1. Definition. We shall say that $U$ is an $\alpha$-domain if

(1) $I=\{x \in \mathrm{R}: u(x)>0\}$ is a bounded, connected interval,

(2) $u$ is smooth on $I$,

(3) $\partial U$ intersects each cylinder $\partial C_{\rho}$ with $0<\rho \leq \alpha$ exactly twice and these intersections are transverse.

Consider an $\alpha$-domain $U$ given by (2.1), and let the endpoints of the interval $I$ be $a_{1}<a_{2}$. 


\section{Mean Curvature Flow Through Singularities for Surfaces of Rotation.}

Condition (2) says that $\partial U$ is a smooth hypersurface, except possibly at its endpoints $\left(a_{1}, 0\right),\left(a_{2}, 0\right)$. By the third condition there exist $\delta_{1}, \delta_{2}>0$ such that

$$
\begin{gathered}
u\left(a_{1}+\delta_{1}\right)=u\left(a_{2}-\delta_{2}\right)=\alpha, \\
u(x)<\alpha \text { for } x \in\left(a_{1}, a_{1}+\delta_{1}\right) \cup\left(a_{2}-\delta_{2}, a_{2}\right) \\
u(x)>\alpha \text { for } x \in\left(a_{1}+\delta_{1}, a_{2}-\delta_{2}\right) .
\end{gathered}
$$

It also follows from the same condition that

$$
u^{\prime}(x) \quad \begin{cases}>0 & \text { when } x \in\left(a_{1}, a_{1}+\delta_{1}\right] \\ <0 & \text { when } x \in\left[a_{2}-\delta_{2}, a_{2}\right)\end{cases}
$$

The inverses of $\left.u\right|_{\left[a_{1}, a_{1}+\delta_{1}\right]}$ and $\left.u\right|_{\left[a_{2}-\delta_{2}, a_{2}\right]}$ are therefore well defined functions $v_{1}, v_{2}$ : $[0, \alpha] \rightarrow \mathrm{R}$. By the inverse function theorem they are smooth on $(0, \alpha]$ and they satisfy:

$$
v_{1}^{\prime}(r)>0, \quad v_{2}^{\prime}(r)<0, \quad(0<r \leq \alpha)
$$

Finally, near the axis, the surface $\partial U$ is the union of two graphs:

$$
\partial U \cap C_{\alpha}=\left\{(x, y): 0 \leq r \leq \alpha, x=v_{i}(r),(i=1,2)\right\}
$$

We shall refer to the two components of $\partial U \cap C_{\alpha}$ as the left and right caps of $\partial U$.

Figure 2.1-A typical $\alpha$-domain.

Graph equations. Let $v(t, y)$ be some smooth function on an open subset of $\mathrm{R} \times \mathrm{R}^{n}$, then the graph $\Gamma_{t}$ of $v(t, \cdot)$ is a hypersurface in $\mathrm{R}^{n+1}$. This family of hypersurfaces moves by its mean curvature if and only if

$$
v_{t}=g^{i j}\left(v_{x_{1}}, \ldots, v_{x_{n}}\right) v_{x_{i} x_{j}} .
$$

Here the $g^{i j}$ denote the components of the inverse matrix to $\left[g_{i j}\right]$, and $g_{i j}$ are the components of the metric on $\Gamma_{t}$ in the coordinates $x_{1}, \ldots, x_{n}$. Note that we are using the summation convention to conserve notation. The $g^{i j}$ are given by

$$
g^{i j}\left(p_{1}, \ldots, p_{n}\right)=\frac{\left(1+|p|^{2}\right) \delta^{i j}-p_{i} p_{j}}{1+|p|^{2}}
$$

We shall refer to equation (2.6) as the full graph equation. 


\section{Altschuler, Angenent and Giga}

If $\Gamma_{t}$ is a family of rotationally symmetric hypersurfaces, then parts of $\Gamma_{t}$ may be represented either as horizontal graphs, $r=u(x, t)$, or as vertical graphs, $x=v(r, t)$.

If $\Gamma_{t}$ is given as a horizontal graph, then $\Gamma_{t}$ evolves by its mean curvature iff $u$ satisfies the horizontal graph equation

$$
\frac{\partial u}{\partial t}=\frac{u_{x x}}{1+u_{x}^{2}}-\frac{n-1}{u} .
$$

If $\Gamma_{t}$ is given as a vertical graph, then $\Gamma_{t}$ evolves by its mean curvature iff $v$ satisfies the vertical graph equation

$$
\frac{\partial v}{\partial t}=\frac{v_{r r}}{1+v_{r}^{2}}+\frac{n-1}{r} v_{r} .
$$

In general, if $\Gamma(t) \subset \mathrm{R}^{n+1}$ is a family of smooth hypersurfaces, obtained by rotating a family $\gamma(t)$ of smooth curves in the upper halfplane around the $x$-axis, then the $\Gamma(t)$ will evolve by their mean curvature if and only if the $\gamma(t)$ evolve with normal velocity given by

$$
v=k+\frac{n-1}{r} \cos \theta,
$$

where $\theta$ denotes the angle between the tangent to $\gamma(t)$ at $(x, r)$ and the $x$-axis, and $k$ is the curvature of $\gamma(t)$ at $(x, r)$. Both the horizontal and vertical graph equations are equivalent to this equation, when they are meaningfull.

The Evans-Spruck estimates. We shall now recall the interior gradient estimates of Evans and Spruck [ES3] and Ecker and Huisken [EH2] which were obtained by adapting Korevaar's [Ko] arguments. These estimates give $a$ priori bounds on $|\nabla v|$ for any solution of the full graph equation.

Let $B_{R}(p)$ be an open ball of radius $R$ centered at $p \in \mathrm{R}^{n}$ and let $T>0, \delta>0, t_{1} \in \mathrm{R}$ be given constants. Assume that the function $v=v(t, y)$ is defined on

$$
Q=(0, T+\delta) \times B_{R}(p) .
$$

2.2. Lemma. Suppose that $v \in C^{3}(Q) \cap C(\bar{Q})$ is a solution of the full graph equation in Q. Then

$$
|\nabla v(T, p)| \leq(3+48 M / R) e^{2 L}
$$

with $L=2+180 M^{2} / T+720 n M^{2} / R^{2}, M=\sup _{Q}|v|$.

This is the same as [ES3, Corollary 5.3] except that we do not assume $\delta=T$. However, the proof in [ES3] actually works without this assumption. 
Mean Curvature Flow Through Singularities for Surfaces of Rotation.

2.3. Corollary. For $s_{1}<s_{2}, \rho>0$ and $q \in \mathrm{R}^{n}$ we set

$$
\Omega=\left(s_{1}, s_{2}\right) \times B_{\rho}(q) .
$$

Suppose that $v \in C^{3}(\Omega)$ solves the full graph equation in $\Omega$ with $M=\sup _{\Omega}|v|<\infty$. For any $\epsilon>0$ there is a constant $C=C(M, \epsilon, n)$ such that

$$
|\nabla v| \leq C \quad \text { on } \quad \Omega_{\epsilon}=\left(s_{1}+\epsilon^{2}, s_{2}\right) \times B_{p-\epsilon}(q) .
$$

Proof. For $\left(t_{1}, p\right) \in \Omega_{\epsilon}$ we put

$$
Q=\left(t_{1}-T, t_{1}+\delta\right) \times B_{R}(p)
$$

with $T=\epsilon^{2}, R=\epsilon$ so that $Q \subset \Omega$ for sufficiently small $\delta$. The estimate (2.10) now yields (2.12).

Q. E. D.

If the gradient is bounded, then by classical interior Hölder gradient and Schauder estimates, one can bound the higher space and time derivatives (see [LUS]). We state a version of such results for the reader's convenience. Note that such results hold for general quasilinear parabolic equations other than the full graph equation.

2.4. Lemma. Let $\Omega$ be as in (2.11). Suppose that $v \in C^{3}(\Omega)$ solves the full graph equation in $\Omega$ with $M=\sup _{\Omega}|v|<\infty$ and $N=\sup _{\Omega}|\nabla v|<\infty$. Then,

(i) For $\epsilon>0$ and nonnegative integer $l$ there is a constant $C^{\prime}=C^{\prime}(M, N, \epsilon, n, l)$ such that

$$
\|v\|_{C^{\prime}\left(\bar{\Omega}_{e}\right)} \leq C^{\prime} .
$$

(ii) (Interior estimates up to initial data). If $v \in C^{2 l+1}\left(\left[s_{1}, s_{2}\right) \times B_{\rho}(q)\right)$ then

$$
\|v\|_{C^{\prime}\left(\bar{\Omega}_{e}^{\prime}\right)} \leq C^{\prime \prime}
$$

with $\Omega_{\epsilon}^{\prime}=\left(s_{1}, s_{2}\right) \times B_{\rho-\epsilon}(q)$ and $C^{\prime \prime}=C^{\prime \prime}(M, N, \epsilon, n, l, K)$ where $K$ is a bound of $\|v(0, \cdot)\|_{C^{21+1}\left(B_{p}(q)\right)}$.

Combining the Corollary and Lemma 2.4 (i), we observe that solutions of the full graph equation immediately become smooth, even if the initial data are singular. 


\section{Altschuler, Angenent and Giga}

\section{§3. The Inner and Outer Evolution.}

In this section we recall various fundamental properties of the weak solutions to the mean curvature problem which were introduced in [CGG1, ES1]. These weak solutions were constructed as level sets of a "viscosity solution" to the levelset equation (3.1). We shall follow the approach of [CGG1].

We first recall the definition of a weak solution of the mean curvature flow given in [CGG1], using a slightly different notation. Let $D$ be an open set in the space-time domain $[0, \infty) \times R^{n+1}$, and let $\Gamma$ be a closed set in $[0, \infty) \times R^{n+1}$ which is disjoint from $D$. Suppose that for some $\alpha>0$ there is a viscosity solution $\psi \in \mathcal{K}_{\alpha}$ of

$$
\psi_{t}-|\nabla \psi| \operatorname{div}\left(\frac{\nabla \psi}{|\nabla \psi|}\right)=0 \text { in }(0, \infty) \times \mathrm{R}^{n+1}
$$

where for $\alpha \leq 0$ we define

$$
\begin{aligned}
\mathcal{K}_{\alpha}=\left\{\psi \in C\left([0, \infty) \times \mathrm{R}^{n+1}\right):\right. \\
\left.\quad(\psi-\alpha) \text { has compact support in }[0, T] \times \mathrm{R}^{n+1} \text { for any } T>0\right\} .
\end{aligned}
$$

If

$$
\begin{aligned}
& \Gamma=\left\{(t, z) \in[0, \infty) \times \mathrm{R}^{n+1}: \psi(t, z)=0\right\} \\
& D=\left\{(t, z) \in[0, \infty) \times \mathrm{R}^{n+1}: \psi(t, z)>0\right\}
\end{aligned}
$$

then we say that $(\Gamma, D)$ is a weak solution of the mean curvature flow problem with initial data $(\Gamma(0), D(0))$. Here, and throughout the paper, $W(t)$ denotes the cross-section at time $t$ of any set $W \subset[0, \infty) \times \mathbf{R}^{n+1}$, i.e.

$$
W(t)=\left\{z \in \mathrm{R}^{n+1}:(t, z) \in W\right\}
$$

Of course

$$
W=\bigcup_{t \geq 0}\{t\} \times W(t)
$$

Also, any solution will be understood to be a viscosity solution.

Figure 3.1-A possible choice of $(U, \Sigma)$. 


\section{Mean Curvature Flow Through Singularities for Surfaces of Rotation.}

Suppose that $U$ is a given bounded open set in $\mathbf{R}^{n+1}$, and that $\Sigma$ is a closed subset of $\mathbf{R}^{n+1}$ which is disjoint from $U$, but which contains $\partial U$. According to [CGG1] there exists a unique weak solution $(\Gamma, D)$ with initial data $(\Gamma(0), D(0))=(\Sigma, U)$ (in fact, such a solution was constructed for a much larger class of geometric initial value problems.) For the mean curvature flow Evans and Spruck [ES1] constructed the set $\Gamma$, given the compact but otherwise arbitrary initial datum $\Gamma(0)$. This indicates that $\Gamma$ is completely determined by $\Gamma(0)$, and that it is independent of $D(0)$. In theorem 3.1 we observe a closely related fact: we show that the open sets $D(t)$ are uniquely determined by $D(0)$.

Intuitively $\{\Gamma(t), t \geq 0\}$ is a family of hypersurfaces which evolves by its mean curvature, and $D(t)$ is the "inside" of $\Gamma(t)$. Evans and Spruck [ES1] observed that this intuition is valid if $\Gamma(0)$ is a smooth hypersurface: they showed that, as long as the classical smooth solution $\Gamma(t)$ exists, it coincides with the weak solution; see also [GG2] where the same is proven for general geometric evolutions. On the other hand they also showed that if $\Gamma(0)$ is a "figure eight" in the plane, the unique (!) solution $\Gamma(t)$ has positive area for any $t>0$, and hence cannot be regarded as a curve in any sense.

3.1. Theorem. Let $(\Gamma, D)$ be a weak solution with initial data $(\Gamma(0), D(0))$. Define $E=D \cup \Gamma$.

(i) $D$ depends only on $D(0)$ and is independent of $\Gamma(0)$,

(ii) $E$ is completely determined by $E(0)=D(0) \cup \Gamma(0)$.

PROOF. We first note that by theorem 6.8 of [CGG1] there exists a unique viscosity solution $\psi \in \mathcal{K}_{\alpha}$ of the levelset equation for any given continuous initial value $\psi(0, z)=$ $a(z)$, which is constant outside of a compact domain in $\mathrm{R}^{n+1}$. Choose $a$ so that

$$
\Gamma(0)=\left\{z \in \mathrm{R}^{n+1}: a(z)=0\right\}, \quad D(0)=\left\{z \in \mathrm{R}^{n+1}: a(z)>0\right\} .
$$

Just as in (3.1-3.4), the corresponding solution $\psi^{a} \in \mathcal{K}_{\alpha}$ of the levelset equation then defines a weak solution $(\Gamma, D)$ to the mean curvature flow, by

$$
\begin{aligned}
& \Gamma=\left\{(t, z) \in[0, \infty) \times \mathrm{R}^{n+1}: \psi^{a}(t, z)=0\right\}, \\
& D=\left\{(t, z) \in[0, \infty) \times \mathrm{R}^{n+1}: \psi^{a}(t, z)>0\right\} .
\end{aligned}
$$

Since any choice of $a$ which satisfies (3.5) will produce the same $(\Gamma, D)$ (see [CGG1, Theorem 7.1]), we may choose our $a$ so that

$$
a(z)=d(z, \partial(D(0)))
$$

holds in $D(0)$, where $d$ denotes Euclidean distance.

We introduce the function $\theta: \mathrm{R} \rightarrow \mathrm{R}$, given by $\theta(s)=s \vee 0=\max (s, 0)$. Since (3.1) is "geometric" in the sense of [CGG1], and $\theta$ is continuous and nondecreasing it follows from Theorem 5.2 of [CGG1] that $\phi(t, z)=\theta\left(\psi^{a}(t, z)\right)$ is also a solution of (3.1) in 


\section{Altschuler, Angenent and Giga}

$(0, \infty) \times \mathrm{R}^{n+1}$. By the uniqueness of the solution $\psi^{a}$, given the initial condition $a$ [CGG1, Theorem 6.7], $\phi(t, z)$ is the unique solution of (3.1) in $\mathcal{K}_{0}$ (defined in (3.2)), with

$$
\phi(0, z)= \begin{cases}d(z, \partial(D(0))) & \text { for } z \in D(0), \\ 0 & \text { otherwise. }\end{cases}
$$

Since $D=\{\psi>0\}=\{\phi>0\}$ it follows that $D$ is completely determined by $D(0)$.

The proof of (ii) parallels that of (i) if we take $\theta(s)=s \wedge 0=\min (s, 0)$.

Q.E. D.

As an aside we point out that the same result has been proved in [GGI, remark 2.4], for the case of periodic boundary conditions.

Definition of Inner and Outer Evolution. If $D_{0} \subset \mathrm{R}^{n+1}$ is a bounded open set, then we can choose any compact set $E_{0} \supset D_{0}$ and construct the weak solution $(\Gamma, D)$ corresponding to $\left(E_{0} \backslash D_{0}, D_{0}\right)$. Theorem 3.1 says that $D$ is independent of the way we choose $E$, so it makes sense to define $D$ to be the inner evolution corresponding to $D_{0}$. Likewise we define $E=D \cup \Gamma$ to be the outer evolution corresponding to $E_{0}$.

The inner evolution at time $t$, i.e. $D(t)$, is always an open set, and the outer evolution at time $t, E(t)$, is a closed set.

3.2. Comparison Lemma. Suppose that $D$ and $D^{\prime}$ are inner evolutions with initial data $D(0), D^{\prime}(0)$, respectively; let $E$ and $E^{\prime}$ be outer evolutions with initial data $E(0), E^{\prime}(0)$.

(i) If $D(0) \subset D^{\prime}(0)$ then $D \subset D^{\prime}$,

(ii) If $E(0) \subset E^{\prime}(0)$ then $E \subset E^{\prime}$,

(iii) If $E(0) \subset D^{\prime}(0)$ then $E \subset D^{\prime}$,

(iv) If $D(0) \subset E^{\prime}(0)$ then $D \subset E^{\prime}$.

The nature of inner and and outer evolutions is such that we shall frequently have to represent open or closed sets as regions where a given function is positive, or nonnegative, respectively. Thus we shall call a function $a \in C\left(\mathrm{R}^{n+1}\right)$ admissible for an open set $D_{0} \subset \mathrm{R}^{n+1}$ if $D_{0}=\left\{z \in \mathrm{R}^{n+1}: a(z)>0\right\}$, and if $a(z)$ is constant and non-positive outside of some compact region in $R^{n+1}$.

Similarly, we shall call a function $a \in C\left(\mathrm{R}^{n+1}\right)$ admissible for a closed set $E_{0} \subset \mathrm{R}^{n+1}$ if $E_{0}=\left\{z \in \mathrm{R}^{n+1}: a(z) \geq 0\right\}$, and if $a(z)$ is constant and strictly negative outside of some compact region in $\mathrm{R}^{n+1}$.

Proof of LEMMA 3.2. We begin with (i). Let $a, a^{\prime}$ be admissible functions for $D(0), D^{\prime}(0)$, respectively. By [CGG1, Lemma 7.2] $a$ and $a^{\prime}$ can be chosen so that $a \leq a^{\prime}$, since $D(0) \subset D^{\prime}(0)$. The comparison theorem for weak solutions of (3.1), [CGG1, Theorem 6.7] then implies that the corresponding weak solutions $\psi^{a}$ and $\psi^{a^{\prime}}$ are also ordered: $\psi^{a} \leq \psi^{a^{\prime}}$. Hence $D \subset D^{\prime}$.

The proof of (ii) may be given along the same lines.

Concerning (iii) we observe that $E(0)$ is a compact subset of the open bounded domain $D^{\prime}(0)$, so that there exists a $a \in C\left(\mathrm{R}^{n+1}\right)$ with 


\section{Mean Curvature Flow Through Singularities for Surfaces of Rotation.}

(*) $a(z) \equiv-1$ outside some large ball which contains $D^{\prime}(0)$,

(**) $D^{\prime}(0)=\left\{z \in \mathrm{R}^{n+1}: a(z)>0\right\}$, and $E(0)=\left\{z \in \mathrm{R}^{n+1}: a(z) \geq 1\right\}$.

Again, there is a unique weak solution $\psi^{a}$ of (3.1) which is constant outside some large ball in space, and for which $\psi^{a}(0, z)=a(z)$. Since $a$ is admissible for $D^{\prime}(0)$ and $a-1$ is admissible for $E(0)$, we see

$$
E=\{(t, z): \psi(t, z) \geq 1\}, \quad D=\{(t, z): \psi(t, z)>0\},
$$

which clearly implies that $E \subset D^{\prime}$. Here we use the fact that with $\psi^{a}$, all functions $\psi^{a}-c$ $(c \in \mathrm{R})$ are also viscosity solutions of (3.1).

Finally, (iv) is trivially true since $(\Gamma, D)$ with $\Gamma=E^{\prime} \backslash D$ is the weak solution to the mean curvature flow problem with initial data $\left(E^{\prime}(0) \backslash D(0), D(0)\right)$.

Q.E. D.

Convergence and Separation Properties. If $\left\{U_{j}: j=1,2, \ldots\right\}$ is a sequence of open sets, then we shall write $U_{j} \uparrow U$ if $U_{j} \subset U_{j+1}$ for all $j \geq 1$, and $U=U_{j \geq 1} U_{j}$. If $\left\{C_{j}: j=1,2, \ldots\right\}$ is a sequence of closed sets, then $C_{j} \downarrow C$ will mean that $C_{j} \supset C_{j+1}$, and $C=\cap_{j \geq 1} C_{j}$.

We begin this subsection with a useful lemma which was implicitely used in [ES3].

3.3. Approximation lemma. Let $D$ be an inner evolution. Then there exist two sequences of inner evolutions $\left\{D_{k}^{\prime}: k \geq 1\right\}$ and outer evolutions $\left\{E_{k}^{\prime}: k \geq 1\right\}$ such that

$$
D_{k}^{\prime} \uparrow D \text { and } D_{k}^{\prime} \subset E_{k}^{\prime} \subset D_{k+1}^{\prime}
$$

Proof. Let $\psi:[0, \infty) \times \mathbf{R}^{n+1} \rightarrow \mathbf{R}$ be a viscosity solution of the levelset equation for which $D$ is the set where $\psi$ is positive. Then we define

$$
D_{k}^{\prime}=\left\{(t, z): \psi(t, z)>2^{-k}\right\}, \quad E_{k}^{\prime}=\left\{(t, z): \psi(t, z) \geq 2^{-k}\right\} .
$$

These sets clearly satisfy (3.6), and since $\psi-2^{-k}$ is a viscosity solution of the levelset equation they are inner and outer evolutions, respectively.

Q. E. D.

\subsection{Monotone Convergence Theorem.}

(i) Let $D$ and $\left\{D_{j}: j=1,2, \ldots\right\}$ be inner evolutions. If $D_{j}(0) \uparrow D(0)$ then $D_{j} \uparrow D$.

(ii) If $E$ and $E_{j}$ are outer evolutions then $E_{j}(0) \downarrow E(0)$ implies $E_{j} \downarrow E$.

Proof. We shall prove the statement about inner evolutions, and leave the analogous proof for outer evolutions to the reader's imagination.

Let $D_{k}^{\prime}, E_{k}^{\prime}$ be the approximating inner and outer evolutions for $D$ which were constructed in the previous lemma. Then $E_{k}^{\prime}(0)$ is a compact subset of $D(0)$, so there is a $j_{k} \geq 1$ such that $E_{k}^{\prime}(0) \subset D_{j_{k}}(0)$. By the comparison theorem we have $E_{k}^{\prime} \subset D_{j_{k}}$. But 


\section{Altschuler, Angenent and Giga}

the sequence $E_{k}^{\prime}$ was constructed so that $D_{k}^{\prime} \subset E_{k}^{\prime} \uparrow D$, and we see that $D_{j_{k}} \uparrow D$, which proves the theorem.

Q. E. D.

All the results which we have stated so far could have been formulated and proved for the more general geometric evolution equations of [GG1] than just the mean curvature flow. In the following result we use a symmetry property of the levelset equation: if $\psi$ is a viscosity solution of (3.1) then so is $-\psi$. Geometrically, this symmetry reflects the fact that the definition of mean curvature flow for smooth surfaces does not involve the orientation of those surfaces. The viscosity solution approach introduces an orientation of the evolving hypersurface since we regard it as the boundary of what is "inside" the surface.

3.5. Separation theorem. Let $D_{i}$ and $E_{i}$ be inner and outer evolutions, respectively $(i=1,2)$.

(i) $d\left(E_{1}(t), E_{2}(t)\right) \geq d\left(E_{1}(0), E_{2}(0)\right)$ for all $t \geq 0$.

(ii) $d\left(D_{1}(t), D_{2}(t)\right) \geq d\left(D_{1}(0), D_{2}(0)\right)$ for all $t \geq 0$.

(iii) If $D_{1}(0)$ and $D_{2}(0)$ are disjoint then so are $D_{1}$ and $D_{2}$.

(iv) Let $D$ be the inner evolution with initial datum $D_{1}(0) \cup D_{2}(0)$. If $D_{1}(0)$ and $D_{2}(0)$ are disjoint then $D=D_{1} \cup D_{2}$.

The first two statements in this theorem are well known for smooth solutions of the mean curvature flow (e.g. [A1, Il], and in particular Matt Grayson's $\delta$-whiskers [GrI]), while it is also known that they do not hold for the more general situation in which one evolves objects of high codimension by their mean curvature vectors (see [Alt].)

This theorem will turn out to be useful because of the last two statements. The reason for this is that they still apply when the two sets $D_{1}(0)$ and $D_{2}(0)$ are disjoint, but have zero distance, i.e. when their closures intersect.

PROOF. The proofs of (i) and (ii) are very similar, and the proof of (i) is essentially given in [ES1], so we shall only prove (ii), for completness' sake.

Let $d$ be the distance between $D_{1}(0)$ and $D_{2}(0)$, and write $d_{j}(z)$ for the distance from $z \in \mathbf{R}^{n+1}$ to $\partial\left(D_{j}(0)\right)(j=1,2)$. Define

$$
a(z)= \begin{cases}d_{1}(z)+d / 2 & \text { for } z \in D_{1}(0) \\ -d_{2}(z)-d / 2 & \text { for } z \in D_{2}(0) \\ \left(d_{2}(z) \wedge \frac{d}{2}\right)-\left(d_{1}(z) \wedge \frac{d}{2}\right) & \text { otherwise. }\end{cases}
$$

We claim that $a$ is Lipschitz with constant 1 . On the open sets $D_{i}(0)(i=1,2)$ this is clear, since the distance to a set is always Lipschitz with constant 1. Outside of the closures of the $D_{i}(0)$ 's we always have $d_{1}(z)+d_{2}(z) \geq d$, by the triangle inequality, so the open sets

$$
V_{i}=\left\{z \in \mathrm{R}^{n} \backslash\left(\overline{D_{1}(0)} \cup \overline{D_{2}(0)}\right): d_{i}(z)<d / 2\right\}
$$

are disjoint. On these sets $a(z)$ is given by $\pm\left(d_{i}(z)-d / 2\right)$, so that $a$ is Lipschitz with constant 1 on these sets too. Finally, $a(z)$ vanishes on the complement of $D_{1}(0) \cup D_{2}(0) \cup$ 
$V_{1} \cup V_{2}$, and by piecing these separate Lipschitz estimates together one finds that $a$ is Lipschitz with constant 1 on all of $\mathrm{R}^{n+1}$.

Moreover, $a$ vanishes far away from $D_{1}(0) \cup D_{2}(0)$.

Let $\psi \in \mathcal{K}_{0}$ denote the solution of the levelset equation with initial value $a$. Then $\pm \psi+c$ is also a solution for any $c \in \mathrm{R}$. This implies that the inner evolution corresponding to the set $D^{\prime}(0)=\left\{z \in \mathrm{R}^{n+1}: a(z)<\beta\right\}$ is $D^{\prime}=\left\{(t, z) \in[0, \infty) \times \mathrm{R}^{n+1}: \psi(t, z)<\beta\right\}$, whatever the value of $\beta<0$. Applying this observation to

$$
D_{1}(0)=\left\{z \in \mathrm{R}^{n+1}: a(z)>d / 2\right\}, \quad D_{2}(0)=\left\{z \in \mathrm{R}^{n+1}: a(z)<-d / 2\right\},
$$

we find

$$
\begin{gathered}
D_{1}=\left\{(t, z) \in[0, \infty) \times \mathbf{R}^{n+1}: \psi(t, z)>d / 2\right\}, \\
D_{2}=\left\{(t, z) \in[0, \infty) \times \mathbf{R}^{n+1}: \psi(t, z)<-d / 2\right\} .
\end{gathered}
$$

This clearly implies that $D_{1}$ and $D_{2}$ are disjoint.

The levelset equation (3.1) is invariant under translations, and under the substitution $\psi \mapsto \psi+c$. Using a comparison principle this allows one to prove (cf. [ES1, GGIS]) that the Lipschitz property of $a$ implies that each $\psi(t, \cdot)$ is a Lipschitz function on $\mathrm{R}^{n+1}$, with constant $\leq 1$. Thus if $z_{j} \in D_{j}(t)$ then by (3.8)

$$
d=d / 2+d / 2<\psi\left(t, z_{1}\right)-\psi\left(t, z_{2}\right) \leq\left|z_{1}-z_{2}\right|
$$

which completes the proof of (ii).

To prove (iii) we simply observe that the preceding argument still works if $d=0$. It allows us to derive $(3.7,8)$ and hence the disjointness of $D_{1}, D_{2}$.

To prove (iv) we invoke the approximation lemma to construct two sequences of inner and outer evolutions

$$
D_{1}^{j} \subset E_{1}^{j} \subset D_{1}^{j+1} \text { and } D_{2}^{j} \subset E_{2}^{j} \subset D_{2}^{j+1} \text {. }
$$

Let $D^{j}$ be the inner evolution with initial value $D_{1}^{j}(0) \cup D_{2}^{j}(0)$. Since $E_{1}^{j}$ and $E_{2}^{j}$ are compact $\delta^{j}=d\left(E_{1}^{j}, E_{2}^{j}\right)>0$, and thus $d\left(D_{1}^{j}, D_{2}^{j}\right) \geq d\left(E_{1}^{j}, E_{2}^{j}\right)>0$. This implies that $D^{j}=D_{1}^{j} \cup D_{2}^{j}$; indeed, if $0 \leq \psi_{1}^{j}, \psi_{2}^{j} \in C\left([0, \infty) \times \mathrm{R}^{n+1}\right)$ are viscosity solutions of the levelset equation with $D_{m}^{j}=\left\{(t, z): \psi_{m}^{j}>0\right\}$, then $\operatorname{supp} \psi_{1}^{j}$ and $\operatorname{supp} \psi_{2}^{j}$ are disjoint, and separated by at least $\delta^{j}$, so that $\psi^{j}=\psi_{1}^{j} \vee \psi_{2}^{j}$ is again a viscosity solution of the levelset equation; the inner evolution which starts with $D_{1}^{j}(0) \cup D_{2}^{j}(0)$ therefore is given by $\left\{(t, z): \psi^{j}>0\right\}=D^{j}$.

We have constructed a sequence of inner solutions $D^{j}$ which increases to $D_{1} \cup D_{2}$, and whose initial values increase to $D(0)$. By the monotone convergence theorem we have $D^{j} \uparrow D$, and hence $D=D_{1} \cup D_{2}$.

Q. E. D. 


\section{Altschuler, Angenent and Giga}

As functions of time the sections $D(t)$ and $E(t)$ of an inner and outer evolution have a certain continuity. To formulate this continuity we define the $\varepsilon$-core $\mathcal{C}_{\varepsilon}(V)$ of an open set $V \subset \mathrm{R}^{n+1}$ to be

$$
\mathcal{C}_{\varepsilon}(V)=\left\{x \in \mathrm{R}^{n+1}: \operatorname{dist}\left(x, V^{c}\right) \geq \varepsilon\right\}
$$

where $V^{\mathrm{c}}=\mathrm{R}^{n+1} \backslash V$.

This concept is dual to that of an $\varepsilon$-neighbourhood of a closed set in $\mathrm{R}^{n+1}$ : indeed, one has $\mathcal{C}_{\varepsilon}(V)=\left(\mathcal{N}_{\varepsilon}\left(V^{c}\right)\right)^{c}$.

3.6. Continuity in time. Let $D$ and $E$ be inner and outer evolutions.

(i) $D(t)$ is a lower semicontinuous function of $t \in[0, \infty)$, in the sense that for any $t_{0} \geq 0, \varepsilon>0$ as $>0$ can be found such that

$$
\left|t-t_{0}\right|<\delta \Rightarrow D(t) \supset \mathcal{C}_{\varepsilon}\left(D\left(t_{0}\right)\right)
$$

Similarly, $E(t)$ is an upper semicontinuous function:

$$
\forall_{t_{0} \geq 0} \forall_{c}>0 \exists_{\delta>0} \quad\left|t-t_{0}\right|<\delta \Rightarrow E(t) \subset \mathcal{N}_{\varepsilon}\left(E\left(t_{0}\right)\right) .
$$
with

(ii) $D(t)$ is also left upper semicontinuous in $t$, i.e. for any $t_{0}, \varepsilon>0$ a $\delta>0$ exists

$$
t_{0}-\delta<t<t_{0} \Rightarrow \mathcal{C}_{\varepsilon}(D(t)) \subset D\left(t_{0}\right)
$$

and $E(t)$ is left lower semicontinuous in $t$ :

$$
\forall_{t_{0}}>0 \forall_{\varepsilon>0} \exists_{\delta>0} \quad t_{0}-\delta<t<t_{0} \Rightarrow \mathcal{N}_{e}(E(t)) \supset E\left(t_{0}\right) .
$$
by

For any two closed sets $C_{1}, C_{2} \subset \mathrm{R}^{n+1}$ the Hausdorff distance between them is defined

$$
d_{H}\left(C_{1}, C_{2}\right)=\inf \left\{\varepsilon>0: C_{1} \subset \mathcal{N}_{\varepsilon}\left(C_{2}\right) \& C_{2} \subset \mathcal{N}_{\varepsilon}\left(C_{1}\right)\right\} .
$$

One can define a dual metric between open subsets $V_{1}, V_{2} \subset \mathrm{R}^{n+1}$ by

$$
d_{H}^{*}\left(V_{1}, V_{2}\right)=\inf \left\{\varepsilon>0: \mathcal{C}_{\varepsilon}\left(V_{1}\right) \subset V_{2} \& \mathcal{C}_{\varepsilon}\left(V_{2}\right) \subset V_{1}\right\} .
$$

Clearly $d_{H}^{*}\left(V_{1}, V_{2}\right)=d_{H}\left(V_{1}^{\mathrm{c}}, V_{2}^{\mathrm{c}}\right)$.

The theorem implies that $D(t)$ and $E(t)$ are left continuous functions with respect to the Hausdorff metrics on open and closed sets.

Proof. (i) follows easily from the fact that $D, E$ are open and closed sets in $[0, \infty) \times$ $\mathrm{R}^{n+1}$, respectively. E.g. $\mathcal{C}_{\varepsilon}\left(D\left(t_{0}\right)\right)$ is compact, so there is a $\delta>0$ such that $\mathcal{C}_{\varepsilon}\left(D\left(t_{0}\right)\right) \times$ $\left(t_{0}-\delta, t_{0}+\delta\right) \subset D$, which is what is claimed.

The two statements in (ii) are dual to each other, and we shall only deal with the case of inner evolutions. 


\section{Mean Curvature Flow Through Singularities for Surfaces of Rotation.}

Let $\varepsilon>0, t_{0}>0$ be given; put $\delta=\varepsilon^{2} / 2 n$; assume that $t$ is given with $t_{0}-\delta<t<t_{0}$. Given any point $P \in \mathcal{C}_{\varepsilon}(D(t))$ we observe that

$$
U_{\tau}=\left\{(s, Q) \in[0, \infty) \times \mathbf{R}^{n+1}: d(Q, P)^{2}<2 n(\tau-s)\right\} .
$$

is an inner evolution which at time $t$ is a ball of radius $\varepsilon^{\prime}=\sqrt{2 n(\tau-t)}$ centered at $P$. Since $t_{0}-t<\delta=\varepsilon^{2} / 2 n$ there is a $\tau>t_{0}$ such that $\tau-t<\delta$. For this $\tau$ we have $U_{\tau}(t)=B_{\varepsilon^{\prime}}(P)$ with $\varepsilon^{\prime}<\varepsilon$, so that $U_{\tau}(t) \subset D(t)$. By the comparison lemma we then have $U_{\tau}\left(t_{0}\right) \subset D\left(t_{0}\right)$, and hence $P \in D\left(t_{0}\right)$.

Q.E. D.

Regular Evolutions, or The Interior of $\Gamma(t)$. Evans and Spruck's example of the figure eight shows that the set $\Gamma=E \backslash D$ may have an interior, even if the initial value $\Gamma(0)$ has none. In this subsection we shall make some simple observations about the way $\Gamma(t)$ can develop an interior.

3.7. Definition. An inner evolution $D$ is called regular if its closure $\bar{D}$ is an outer evolution.

Suppose that $\Gamma\left(t_{0}\right)$ has an interior for some $t_{0} \geq 0$, say, $\Gamma\left(t_{0}\right)$ contains an $\varepsilon$ neighbourhood of $P$. Then $U\left(t_{0}\right) \subset \Gamma\left(t_{0}\right)$, where $U$ is the inner evolution corresponding to a shrinking ball, centered at $P$, and with radius $\varepsilon$ at time $t_{0}$, i.e. $U=U_{\tau}$ as in (3.9), with $\tau=\varepsilon^{2} / 2 n+t_{0}$. This means that $U\left(t_{0}\right) \subset E\left(t_{0}\right)$ while $U\left(t_{0}\right)$ and $D\left(t_{0}\right)$ are disjoint. By the comparison and separation lemmas we have $U(t) \subset E(t) \backslash D(t)$ for all $t \geq t_{0}$. Hence we have proved the following.

3.8. Lemma. If $\Gamma\left(t_{0}\right)$ has an interior, then so do all $\Gamma(t)$ with $t \in\left[t_{0}, t_{0}+\delta\right)$, for some small enough $\delta>0$.

Indeed, we may take $\delta=\varepsilon^{2} / 2 n$.

We conclude this section with a sufficient condition for the absence of an interior in $\Gamma$.

3.9. Regularity lemma for Monotone Motion. Let $D$ and $E$ be inner and outer evolutions with $D \subset E$.

(i) If $D(\tau) \subset D(0)$ for all sufficiently small $\tau>0$ then $D(t) \subset D(s)$ whenever $t \geq s \geq 0$.

(ii) If $E(\tau) \subset D(0)$ for all sufficiently small $\tau>0$, then $E(t) \subset D(s)$ whenever $t>s \geq 0$, and $\bar{D}=E$ in $(0, \infty) \times \mathrm{R}^{n+1}$.

Proof. Part (i) follows directly from the semigroup property. If we put

$$
D^{\prime}=\{(t-\tau, z):(t, z) \in D\},
$$

then $D^{\prime}$ is the inner evolution with initial datum $D(\tau)$. For sufficiently small $\tau>0$ we have $D^{\prime}(0) \subset D(0)$, so that $D^{\prime} \subset D$. The comparison lemma tells us that $D(t-\tau)=D^{\prime}(t) \subset$ 


\section{Altschuler, Angenent and Giga}

$D(t)$ so that the theorem is true if $t-s$ is small enough. Iteration of this argument then shows that (i) actually holds for all $t \geq s \geq 0$.

A similar argument shows that, under the hypotheses of part (ii), one has $E(t) \subset D(s)$ for all $t>s>0$. In particular we have

$$
E(t) \subset \bigcap_{0<t^{\prime}<t} D\left(t^{\prime}\right) \subset \bigcap_{0<t^{\prime}<t} \bar{D}\left(t^{\prime}\right) .
$$

Since the $\bar{D}\left(t^{\prime}\right)$ are decreasing in $t^{\prime}$, the continuity theorem 3.6(i) implies that $\bar{D}\left(t^{\prime}\right) \downarrow \bar{D}(t)$ as $t^{\prime} \uparrow t$. Hence $E(t) \subset \bar{D}(t)$ for all $t>0$, and $E \subset \bar{D}$ in $(0, \infty) \times \mathbf{R}^{n+1}$. Since the converse inclusion $\bar{D} \subset E$ is true by assumption, this completes the proof of part (ii).

Q. E. D.

3.10. Theorem. Let $D(0)$ be smoothly bounded domain such that $\partial D$ has positive mean curvature everywhere. Denote the inner and outer evolutions with initial data $D(0)$ and $\bar{D}(0)$ by $D$ and $E$, respectively. Then $E(t) \subset D(s)$ for all $t>s \geq 0$, and $\bar{D}=E$.

Indeed, $\partial D(t)$ and $\partial E(t)$ will be the same smooth hypersurface for a short time, while the positivity of the mean curvature guarantees that the normal velocity of $\partial D(t)$ is bounded away from zero, if $t$ is small. This implies that $E(\tau) \subset D(0)$ for sufficiently small $\tau>0$. The theorem then follows immediately from the previous lemma.

The last result of this section provides another sufficient condition for $E=\bar{D}$ which doesn't involve monotone motion. Although its appearance is more technical than the previous conditions, it will enable us to show that rotationally symmetric domains satisfy $\bar{D}=E$.

3.11. Second regularity lemma. Let $U$ be a bounded open set in $\mathrm{R}^{n+1}$ which may be written as the union of a finite number of disjoint open sets $U^{1}, \ldots, U^{k}$. Denote the inner evolutions with initial data $U$ and $U^{i}$ by $D$ and $D^{i}$, respectively $(1 \leq i \leq k)$, let $E^{i}$ be the outer evolutions with initial data $\bar{U}^{i}$, and assume that $E^{i}=\bar{D}^{i}$.

Suppose that we are given a sequence of open covers $\left\{U_{\alpha}^{1}, \ldots, U_{\alpha}^{k}\right\}_{\alpha \geq 1}$ of $U$ which satisfies

(i) $U_{\alpha}^{i} \supset U_{\alpha+1}^{i}$ and $\bar{U}^{i}=\cap_{\alpha \geq 1} \bar{U}_{\alpha}^{i}$ for $i=1,2, \ldots, k$.

(ii) The $U_{\alpha}^{1}, \ldots, U_{\alpha}^{k}$ are pairwise disjoint for each $\alpha \geq 1$.

Finally, let $E$ be an outer evolution for which a double sequence $\left\{t_{\alpha, l}\right\}_{\alpha, l \geq 1}$ exists such that $t_{\alpha, l} \downarrow 0$ as $l \rightarrow \infty$, and

$$
E\left(t_{\alpha, l}\right) \subset U_{\alpha}^{1} \cup \cdots \cup U_{\alpha}^{k}
$$

Then $\bar{D}=E$ in $(0, \infty) \times \mathrm{R}^{n+1}$.

Proof. Let $D_{\alpha}^{i}$ be the inner evolution with initial datum $U_{\alpha}^{i}$, let $D_{\alpha}$ be the inner evolution with initial datum $U_{\alpha}^{1} \cup \cdots \cup U_{\alpha}^{k}$. From the separation lemma 3.5(iv) we obtain $D_{\alpha}=D_{\alpha}^{1} \cup \cdots \cup D_{\alpha}^{k}$. 
Mean Curvature Flow Through Singularities for Surfaces of Rotation.

The comparison lemma 3.2(iii) tells us that

$$
\begin{aligned}
E(t) & \subset D_{\alpha}\left(t-t_{\alpha, l}\right) \\
& \subset D_{\alpha}^{1}\left(t-t_{\alpha, l}\right) \cup \cdots \cup D_{\alpha}^{k}\left(t-t_{\alpha, l}\right) \\
& \subset \bar{D}_{\alpha}^{1}\left(t-t_{\alpha, l}\right) \cup \cdots \cup \bar{D}_{\alpha}^{k}\left(t-t_{\alpha, l}\right)
\end{aligned}
$$

for $t>t_{\alpha, l}$. Using the continuity lemma $3.6(\mathrm{i}, \mathrm{ii})$ we can now let $l \rightarrow \infty$, which yields

$$
E(t) \subset \bar{D}_{\alpha}^{1}(t) \cup \cdots \cup \bar{D}_{\alpha}^{k}(t) \text { for } t>0 .
$$

On the other hand $\bar{D}_{\alpha}^{i}$ is contained in $E_{\alpha}^{i}$, the outer evolution starting from $\bar{U}_{\alpha}^{i}$. Since $\bar{U}_{\alpha}^{i} \downarrow \bar{U}^{i}$ as $\alpha \rightarrow \infty$, we have $E_{\alpha}^{i} \downarrow E^{i}$, where $E^{i}$ is the outer evolution with initial datum $\bar{U}^{i}$.

Letting $\alpha \rightarrow \infty$ in (3.11) we find $E(t) \subset E^{1}(t) \cup \cdots \cup E^{k}(t)$ for all $t>0$, and since we have assumed that $E^{i}=\bar{D}^{i}$, we get

$$
E(t) \subset \bar{D}^{1}(t) \cup \cdots \cup \bar{D}^{k}(t) \text { for } t>0 .
$$

We apply the separation lemma 3.5 once again to get $D=D^{1} \cup \ldots \cup D^{k}$, whence $E(t) \subset \bar{D}(t)$ for all $t>0$, i.e. $E \subset \bar{D}$ in $(0, \infty) \times \mathrm{R}^{n+1}$.

Since we already have the converse inclusion $\bar{D} \subset E$, this completes the proof.

Q. E. D.

Unlike the previous conditions for regularity of the evolution, this lemma can be localized in time. Indeed, if one replaces the hypothesis " $E^{i}=\bar{D}^{i n}$ with

$$
E^{i} \cap[0, T] \times \mathrm{R}^{n+1}=\bar{D}^{i} \cap[0, T] \times \mathrm{R}^{n+1}
$$

for some $T>0$, then the same proof will lead to the conclusion that

$$
E \cap(0, T) \times \mathrm{R}^{n+1}=\bar{D} \cap(0, T) \times \mathrm{R}^{n+1} .
$$

It is this localized version which we shall use in section 7.

Besides the sufficient conditions for $E=\bar{D}$ which we have given here another condition is known. H. M. Soner [So] has found a generalization of the positive mean curvature condition for the initial domain $D(0)$ which ensures that $\Gamma$ has no interior.

\section{$\S 4$. Intersections and the Sturmian theorem.}




\section{Altschuler, Angenent and Giga}

Sturm's classical result. The Sturmian theorem states that the number of zeroes (counted with multiplicity) of a solution of a linear parabolic equation of the type

$$
u_{t}=a(x, t) u_{x x}+b(x, t) u_{x}+c(x, t) u
$$

does not increase with time, provided $u$ is defined on a rectangle $x_{0} \leq x \leq x_{1}, 0<t<T$, and $u\left(x_{j}, t\right) \neq 0$ for $j=0,1$, and all $t \in(0, T)$. This result also holds for the number of sign changes, rather than the number of zeroes of $u(\cdot, t)$. If all zeroes of $u(\cdot, t)$ are simple, then the number of zeroes and the number of sign changes coincide of course. It is sometimes advantageous to consider sign changes rather than zeroes, in particular when the coefficients of the equation lack smoothness; in this situation it can still be shown that the number of sign changes does not increase, even though it is not clear whether one can guarantee that this number is always finite (see [Ma]).

The way in which we use the Sturmian theorem in this section is similar to what was done in [A2]. It is also similar to the arguments used by V. A. Galaktionov in a series of papers under the heading "intersection comparison method." We refer to [GP] and the references given there.

The first theorem one can prove using the Sturmian theorem is a direct analog of theorem 1.1 of [A2]. It concerns the intersections of two families of compact smooth hypersurfaces of rotation, $\Gamma_{1}(t)$ and $\Gamma_{2}(t)$, which evolve by their mean curvatures. If two such hypersurfaces are transverse, then their intersection will consist of a finite number of $(n-1)$-spheres. In general, the intersection of two such hypersurfaces will be the union of a possibly infinite number of $(n-1)$-spheres. We shall refer to this number as the number of intersections of $\Gamma_{1}(t)$ and $\Gamma_{2}(t)$.

4.1. Theorem on Intersections. Either $\Gamma_{1}(t) \equiv \Gamma_{2}(t)$ for all $t \in(0, T)$, or the number of intersections of $\Gamma_{1}(t)$ and $\Gamma_{2}(t)$ is finite for all $t \in(0, T)$. In the second case this number is nonincreasing in time, and decreases whenever $\Gamma_{1}(t)$ and $\Gamma_{2}(t)$ have a non-transverse intersection.

This theorem also holds if $\Gamma_{1}$ and $\Gamma_{2}$ have a boundary, provided the $\partial \Gamma_{j}(t)$ are disjoint for $0<t<T$. In fact the theorem even holds for noncompact surfaces under the assumption that the intersection as a subset of space time is compact.

The proof of this theorem can be given along the same lines as that of theorem 1.i in [A2]. It should be noted that it is not necessary to assume that either $\Gamma_{1}(t)$ or $\Gamma_{2}(t)$ are obtained by rotating graphs around the $x$-axis: indeed, when interpreted apropriately it also holds for immersed rather than imbedded solutions to the mean curvature flow. However, in this paper we shall never use the theorem in that generality.

Throughout this section we shall consider a smooth family of surfaces $\Gamma(t)(0 \leq t<$ $T$ ) which evolve by their mean curvature. We shall assume that each $\Gamma(t)$ is compact, connected, and that it is obtained by rotating the graph of a function

$$
r=u(x, t), \quad a(t) \leq x \leq b(t), 0 \leq t<T
$$

around the $x$-axis. 


\section{Mean Curvature Flow Through Singularities for Surfaces of Rotation.}

4.2. Attracting Axis Theorem. For any $\tau>0$ there is a $\delta=\delta(\Gamma(0), \tau)>0$ such that $H>0$ at any point on $\Gamma(t)$ with $r<\delta$ and $t>\tau$.

The third main result says that graph-like surfaces of rotation remain graph-like under flow by mean curvature. This follows rather easily by counting the number of intersections of the given solution with hyperplanes $x=$ const. A more refined argument leads to a quantitative version of the result, i.e. it provides us with an interior gradient estimate for smooth solutions of the horizontal graph equation.

\subsection{Theorem.}

(a) Let $\Gamma(t), 0 \leq t<T$ be a family of smooth hypersurfaces evolving by their mean curvature. If $\Gamma(0)$ is obtained by rotating the graph of a function around the $x$-axis, then so are the $\Gamma(t)$ for $0<t<T$.

(b) Let $\Gamma(t)$ be as in (a), and assume that it is given by a smooth solution of the horizontal graph equation $r=u(x, t)$, with $0<t<T$, and $a(t)<x<b(t)$, for certain smooth functions $a, b:(0, T) \rightarrow \mathbf{R}$. Then there is a function $\sigma: \mathbf{R}_{+} \times \mathbf{R}_{+} \rightarrow \mathbf{R}$ such that

$$
\left|u_{x}(x, t)\right| \leq \sigma(t, u(x, t))
$$

holds for all $0<t<T, a(t)<x<b(t)$. The function $\sigma$ only depends on sup $u(x, 0)$.

We shall see that the function $\sigma$ is of the form $\sigma(t, u)=e^{\rho(u) / t}$, for some positive continuous function $\rho$ on $R_{+}$. It is clear we may also assume that $\sigma(t, u)$ is nonincreasing in $u$ and $t$.

Since the function $u(x, t)$ satisfies the graph equation for $a(t)<x<b(t), 0<t<T$, theorem 4.3 implies a priori estimates for $u_{x}$ and hence (see e.g. [LUS]) for all higher derivatives of $u$, in the interior of the region where $u>0$.

The Catenoid and its generalizations. For the proof of the attracting axis theorem we need to remind the reader of the steady solutions of the horizontal graph equation, i.e. of the minimal surfaces of revolution. These surfaces are obtained by rotating the graph $r=u(x)$ of a solution of

$$
\frac{u_{x x}}{1+\left(u_{x}\right)^{2}}-\frac{n-1}{u}=0
$$

around the $x$-axis. This equation can be integrated once, after multiplying left and right hand sides with $u_{x}$; one finds after some calculations that

$$
u^{\prime}(x)^{2}=\left(\frac{u}{R}\right)^{2(n-1)}-1
$$

for some constant $R>0$. In the case of two dimensional surfaces in $R^{3}$ one can integrate again, and one finds

$$
u(x)=R \cosh \left(\frac{x}{R}\right)
$$




\section{Altschuler, Angenent and Giga}

i.e. one finds the well known family of catenoids. If $n \geq 3$, then it seems that one cannot integrate (4.2) in terms of elementary functions ( $n=3$ can be integrated using elliptic functions.) We shall denote the one parameter family of solutions of (4.2) which arises by

$$
u(x)=R U_{n}\left(\frac{x}{R}\right)
$$

for $n \geq 3$, and we put $\mathcal{U}_{2}(x)=\cosh (x)$.

Unlike the catenoid, the higher dimensional minimal surfaces of rotation have finite width, given by $R \cdot W_{n}$, where

$$
\begin{aligned}
W_{n} & =\int_{1}^{\infty} \frac{d \rho}{\sqrt{\rho^{2(n-1)}-1}} \\
& =\frac{1}{2(n-1)} \mathrm{B}\left(\frac{n-2}{2(n-1)}, \frac{1}{2}\right) .
\end{aligned}
$$

(B is Euler's Beta function.)

Thus $\mathcal{U}_{n}(x)$ is only defined for $|x|<W_{n}$ when $n \geq 3$.

Figure 4.1-The Catenoid \&Co.

We begin with an application of the Sturmian theorem which will lead us to a proof of theorem 4.3, and which will also be useful in the proof of theorem 4.2. The lemma essentially says that the endpoints of the surface $\Gamma(t)$, i.e. the points where it meets the $x$-axis move monotonically.

\subsection{Moving Caps Lemma. $a^{\prime}(t)>0>b^{\prime}(t)$ for $0<t<T$.}

ProOF. Near the point $(a(t), 0)$ on the $x$-axis we can write the surface $\Gamma(t)$ as a vertical graph $x=v(r, t)$, where $v$ is a smooth solution of the vertical graph equation, with $v_{r}(0, t)=0$.

At any time $t_{0} \in(0, T)$ we have

$$
v\left(r, t_{0}\right) \geq v\left(0, t_{0}\right)=a\left(t_{0}\right)
$$

which implies $v_{r r}\left(0, t_{0}\right) \geq 0$, and hence $a^{\prime}\left(t_{0}\right)=n \cdot v_{r r}\left(0, t_{0}\right) \geq 0$.

To prove strict inequality we argue by contradiction, and assume that $v_{r r}\left(0, t_{0}\right)=0$. In this situation $w(r, t) \equiv v(r, t)-a\left(t_{0}\right)$ is a smooth solution of the vertical graph equation, defined for $(r, t)$ near $\left(0, t_{0}\right)$, which satisfies $w\left(0, t_{0}\right)=w_{r}\left(0, t_{0}\right)=w_{r r}\left(0, t_{0}\right)=0$. 


\section{Mean Curvature Flow Through Singularities for Surfaces of Rotation.}

The arguments in sections 3 and 4 of [A4] (which essentially present the Sturmian theorem for equations with a singular first order coefficient) imply that there is some integer $k>1$ such that

$$
\partial_{r}^{j} w\left(0, t_{0}\right) \begin{cases}=0 & \text { for } j<2 k, \\ \neq 0 & \text { when } j=2 k .\end{cases}
$$

Moreover, when $t<t_{0}$ is close to $t_{0}, w(\cdot, t)$ must have $k \geq 2$ zeroes. This is absurd however, since it would mean that $\Gamma(t)$ intersected the plane $x=a\left(t_{0}\right)$ at least $k$ times, while it is obtained by rotating a graph of the form $r=u\left(x, t_{0}\right)$ around the $x$-axis.

Thus $a^{\prime}\left(t_{0}\right)>0$ and it is clear that the same argument can be used to prove $b^{\prime}\left(t_{0}\right)<0$. Q. E. D.

Figure 4.2-The absurd situation in the proof of lemma 4.4.

Proof of Theorem 4.3.a. Let $\Gamma(0)$ be smooth, and suppose it is obtained by rotating the graph of $r=u(x), a<x<b$ around the $x$-axis. Consider any $x \in(a, b)$ and let $\pi_{0} \subset \mathbf{R}^{n+1}$ be the hyperplane given by $x \equiv x_{0}$. Initially $\pi_{0}$ and $\Gamma(t)$ have only one intersection. If we could apply the intersection theorem then it would follow that $\pi_{0}$ and $\Gamma(t)$ have at most one intersection for any $t \in(0, T) ; x_{0}$ being arbitrary then implies that $\Gamma(t)$ is indeed graphlike.

To complete the proof we now show that $\# \pi_{0} \cap \Gamma(t)$ does not increase with time. If $\pi_{0}$ and $\Gamma\left(t_{0}\right)$ are transverse, then $\# \pi_{0} \cap \Gamma(t)$ does not change as $t$ increases beyond $t_{0}$. If $\pi_{0}$ and $\Gamma(0)$ have a nontransverse intersection then $\Gamma(t)$ may be represented as a vertical graph $x=v(r, t)$ near this intersection. The intersections of $\pi_{0}$ and $\Gamma(t)$ then correspond to zeroes of $v(\cdot, t)-x_{0}$, transverse intersections corresponding to simple zeroes.

The hypersurfaces $\Gamma(t)$ are real analytic, since they are locally graphs of solutions of the graph equation. Hence $v(\cdot, t)$ is real analytic, and $v(\cdot, t)-x_{0}$ has only isolated zeroes. If the nontransverse intersection is not on the $x$-axis, then it corresponds to a multiple zero of $v\left(\cdot, t_{0}\right)-x_{0}$ at some $r_{0}>0$. The Sturmian theorem applied to $v(\cdot, t)-x_{0}$ implies that its number of zeroes near $\left(r_{0}, t_{0}\right)$ does not increase. Should the nontransverse intersection occur on the $x$-axis, then we appeal to the arguments in [A4, 33 and 4] again, to arrive at the same conclusion.

Q. E. D.

Proof of Theorem 4.3.b. Let $N=\sup _{x \in \mathrm{R}} u(x, 0)$, or $N=5$, whichever choice leads to the biggest number. It follows from the horizontal graph equation (or by comparing with a shrinking cylinder) that $u(x, t) \leq N$ for $0<t<T, a(t)<x<b(t)$. 


\section{Altschuler, Angenent and Giga}

We shall construct a special solution of the horizontal graph equation. Choose a function $\eta \in C^{\infty}(\mathrm{R})$ such that

$$
\eta(t)= \begin{cases}0 & \text { for } t \leq N+1 \\ -1 & \text { for } t \geq N+2\end{cases}
$$

while $\eta^{\prime}(t) \leq 0$ for all $t \in \mathrm{R}$, and $\eta^{\prime}(t)<0$ for $N+1<t<N+2$. Let $\Gamma$ be the hypersurface given by

$$
\Gamma=\left\{\left(x, y_{1}, \ldots, y_{n}\right) \in \mathrm{R}^{n+1}: x=-\eta(r)\right\},
$$

and denote the corresponding solution to the mean curvature flow equation by $\Gamma(t)$. Since the initial surface $\Gamma$ is the graph of a smooth Lipschitz function, it follows from the results in [EH1] (or even from those in [LUS]) that each $\Gamma(t)$ is also a graph of the form

$$
x=w(r, t)
$$

where $w$ is a smooth solution of the vertical graph equation with initial data $w(r, 0)=-\eta(r)$ and boundary condition $w_{r}(0, t)=0$.

By the maximum principle we have $0<-w(r, t)<1$ for all $r, t>0$. In addition $w_{r} \geq 0$ is bounded, and $\sup _{r \geq 0} w_{r}(r, t)$ is nonincreasing in time. It follows from classical estimates for parabolic PDEs that all derivatives of $w$ are uniformly bounded for $r, t \geq 0$.

By differentiating the vertical graph equation with respect to $r$, one finds that $p(r, t)=$ $w_{r}(r, t)$ satisfies a linear parabolic equation

$$
p_{t}=a(r, t) p_{r r}+b(r, t) p_{r}+c(r, t) p
$$

The coefficients $a, b$ and $c$ of this equation are smooth functions of $r, t>0$. Since $p(r, 0)$ is positive in $N+1<r<N+2$, it follows that for each $\delta>0$ there is a constant $A_{\delta}<\infty$ such that

$$
p(r, t) \geq e^{-A_{\delta} / t}
$$

for all $\delta \leq r \leq N$ and all $0<t<T$.

Let $\Gamma_{\xi}(t)$ denote the translate along the $x$-axis of $\Gamma(t)$ given by

$$
x=w(r, t)+\xi
$$

Since $w_{r}>0$ for all $t>0$ we can also represent $\Gamma_{\xi}(t)$ by $r=v(x-\xi, t)$ for some function $v(x, t)$.

Consider some $0<t_{0}<T$ and $a\left(t_{0}\right)<x_{0}<b\left(t_{0}\right)$. Then there is a unique $\xi \in \mathrm{R}$ with $v\left(x_{0}-\xi, t_{0}\right)=u\left(x_{0}, t_{0}\right)$. 
Figure 4.9-Estimating $u_{x}$.

By the Sturmian theorem the graphs of $u(x, t)$ and $v(x-\xi, t)$ cannot have less intersections when $t<t_{0}$ than they have when $t=t_{0}$.

As $t \downarrow 0$ the graph of $v(x-\xi, t)$, i.e. $\Gamma_{\xi}(t)$ converges to $\Gamma_{\xi}(0)$; this hypersurface intersects the graph of $u(x, 0)$ exactly once so that the graphs of $v\left(x-\xi, t_{0}\right)$ and $u\left(x, t_{0}\right)$ also intersect once. This implies that

$$
u_{x}\left(x_{0}, t_{0}\right) \geq-v_{x}\left(x_{0}, t_{0}\right),
$$

for if this were not the case, then $v(x, t) \uparrow \infty$ as $x \uparrow \xi$ and $v(x, t) \downarrow 0$ as $x \downarrow w(0, t)$, together with $u(a(t), t)=0$ would imply that the graphs of $u\left(x, t_{0}\right)$ and $x\left(x-\xi, t_{0}\right)$ intersect at least twice.

Observing that $v_{x}=1 / w_{r}$ we now conclude from (4.3) and (4.4) that

$$
u_{x}\left(x_{0}, t_{0}\right) \geq \exp \left(\frac{A_{\delta}}{t_{0}}\right) \text {. }
$$

By considering the reflections $\tilde{\Gamma}=\{(x, y): x=\eta(r)\}$ instead of $\Gamma$ one obtains the same bound for $-u_{x}\left(x_{0}, t_{0}\right)$.

Q.E. D.

Proof of Theorem 4.2. Let $\tau>0$ be given. By the moving caps lemma we know that $a^{\prime}(\tau)>0$. Near the point $(a(\tau), 0)$ we can represent $\Gamma(\tau)$ as a graph $x=w(r, t)$, where $w(r, t)$ satisfies the vertical graph equation.

Since $\Gamma(t)$ evolves by its mean curvature we have $0<a^{\prime}(\tau)=H_{(a(\tau), 0)}=n \cdot w_{r r}(0, \tau)$. The continuity of $w_{r r}$ implies that $w_{r r}(r, \tau)>0$ for $0 \leq r \leq \rho$, for some constant $\rho>0$.

The same arguments applied to the other endpoint of $\Gamma(t),(b(t), 0)$, show that $\Gamma(t)$ can be represented by a graph $x=\tilde{w}(r, t)$ near $(b(t), 0)$, and that $\tilde{w}_{r r}(r, \tau)<0$ for $0 \leq r \leq \rho$, provided $\rho>0$ is small enough; $\tilde{w}$ also satisfies the vertical graph equation.

We shall assume that $\rho$ has been chosen so small that the only points on $\Gamma(\tau)$ with $r \leq \rho$ are contained in the two caps $x=w(r, t)$ and $x=\tilde{w}(r, t)$; in other words, we assume that $\Gamma(\tau)$ is a $\rho$-domain.

Figure 4.4-Proving monotonicity near the axis. 
Define

$$
\begin{aligned}
& M=\sup \left\{\left|u_{x}(x, \tau)\right|: u(x, \tau) \geq \rho\right\}, \quad \varepsilon=\left(1+M^{2}\right)^{-1 / 2(n-1)}, \\
& \mathcal{U}_{\delta, \xi}(x)=\delta \mathcal{U}_{n}\left(\frac{x-\xi}{\delta}\right) \quad \text { for } \delta>0, \xi \in \mathbf{R}, \\
& c(\tau)=w(\rho, \tau), \quad d(\tau)=\tilde{w}(\rho, \tau) .
\end{aligned}
$$

4.5. Lemma. If $0<\delta<\varepsilon \rho$ then the graphs of $\mathcal{U}_{\delta, \xi}$ and $u(\cdot, \tau)$ have at most two intersections.

Proof. If $\delta<\varepsilon \rho$ then it follows from (4.2) that whenever $\mathcal{U}_{\delta, \xi} \geq \rho$ we have

$$
U_{\delta, \xi}^{\prime}(x)^{2}=\left(\frac{U_{\delta, \xi}(x)}{\delta}\right)^{2(n-1)}-1>\left(\frac{\rho}{\varepsilon \rho}\right)^{2(n-1)}-1=M^{2} .
$$

From this it follows that the two branches of $\mathcal{U}_{\delta, \xi}(x)$ to the left and right of its symmetry point can intersect the graph of $u(\cdot, \tau)$ at most once in the region $c \leq x \leq d$. Thus if the graph of $\mathcal{U}_{\delta, \xi}$ does not intersect the two caps

$$
\{r=u(x, t): a(\tau) \leq x \leq c(\tau) \text { or } d(\tau) \leq x \leq b(\tau)\}
$$

then it has at most two intersections with the graph of $u(\cdot, \tau)$.

So consider the case in which the graph of $\mathcal{U}_{\delta, \xi}$ intersects one of the caps, say "the one on the left," containing $(a(\tau), 0)$.

Since $\mathcal{U}_{\delta, \xi}$ is convex and $u(\cdot, \tau)$ is concave on the interval $(a(\tau), c(\tau)), \mathcal{U}_{\delta, \xi}$ can intersect the left cap at most twice. If it has two intersections with the left cap, say at $a(\tau)<x_{1}<$ $x_{2}<c(\tau)$, then

and

$$
\mathcal{U}_{\delta, \xi}(x) \geq u(x, \tau) \text { for } x_{2} \leq x \leq c(\tau)
$$

$$
\mathcal{U}_{\delta, \xi}^{\prime}(x)>M \geq\left|u_{x}(x, \tau)\right| \text { for } c(\tau) \leq x \leq d(\tau),
$$

so that $\mathcal{U}_{\delta, \xi}>u(\cdot, \tau)$ for $x_{2} \leq x \leq d(\tau)$; when $x \geq d(\tau)$ we have $u(x, \tau) \leq \rho<\mathcal{U}_{\delta, \xi}(x)$. In this case we may therefore conclude that $\mathcal{U}_{\delta, \xi}$ and $u(\cdot, \tau)$ intersect at most twice. (Note: if $n \geq 3$ then $\mathcal{U}_{\delta, \xi}(x)$ may not be defined for all $x \leq d(\tau)$, but the conclusion remains.)

We must consider one other possibility: namely, that $\mathcal{U}_{\delta, \xi}$ intersects the left cap exactly once, say in $x_{1} \in(a(\tau), c(\tau))$.

Then $\mathcal{U}_{\delta, \xi}(x)>u(x, \tau)$ for $x<x_{1}$, and $\mathcal{U}_{\delta, \xi}^{\prime}\left(x_{1}\right) \leq u_{x}\left(x_{1}, \tau\right)$. First assume that $\mathcal{U}_{\delta, \xi}^{\prime}\left(x_{1}\right)<u_{x}\left(x_{1}, \tau\right)$, and hence that there is at least one other point at which $\mathcal{U}_{\delta, \xi}$ and $u(\cdot, \tau)$ coincide. Using the convexity of $\mathcal{U}_{\delta, \xi},(4.5)$ and the concavity of the two caps one can show that $\mathcal{U}_{\delta, \xi}$ and $u(\cdot, \tau)$ intersect at most once when $x_{1}<x<b(\tau)$, so that they intersect at most twice in total.

If $\mathcal{U}_{\delta, \xi}^{\prime}\left(x_{1}\right)=u_{x}\left(x_{1}, \tau\right)$ then convexity of $\mathcal{U}_{\delta, \xi}$ and concavity of the left cap imply that $\mathcal{U}_{\delta, \xi}(x) \geq u(x, \tau)$ for $x_{1} \leq x \leq c(\tau)$. For $x \geq c(\tau)$ one uses (4.5) to obtain the same conclusion. In this case we therefore also have at most two intersections.

Q.E. D. 


\section{Mean Curvature Flow Through Singularities for Surfaces of Rotation.}

We can now complete the proof of theorem 4.2. Let $\left(x_{0}, t_{0}\right)$ be given, with $t_{0} \geq$ $\tau, a(\tau)<x_{0}<b(\tau)$ and $u\left(x_{0}, t_{0}\right)<\varepsilon \rho$.

Choose $\xi, \delta$ so that

$$
\mathcal{U}_{\delta, \xi}\left(x_{0}\right)=u\left(x_{0}, t_{0}\right), \quad \mathcal{U}_{\delta, \xi}^{\prime}\left(x_{0}\right)=u_{x}\left(x_{0}, t_{0}\right),
$$

i.e. so that the graphs of $\mathcal{U}_{\delta, \xi}$ and $u\left(\cdot, t_{0}\right)$ are tangent at $\left(x_{0}, u\left(x_{0}, t_{0}\right)\right)$. It then follows from (4.2) that

$$
\delta=u\left(1+u_{x}^{2}\right)_{\left(x_{0}, t_{0}\right)}^{-1 / 2(n-1)}<\varepsilon \rho .
$$

Hence we have just proved that the graphs of $\mathcal{U}_{\delta, \xi}$ and $u(\cdot, \tau)$ intersect at most twice. By the theorem on intersections the same is true for the graphs of $U_{\delta, \xi}$ and $u\left(\cdot, t_{0}\right)$.

This implies that $\mathcal{U}_{\delta, \xi}^{\prime \prime}\left(x_{0}\right) \geq u_{x x}\left(x_{0}, t_{0}\right)$, for if $\mathcal{U}_{\delta, \xi}^{\prime \prime}\left(x_{0}\right)<u_{x x}\left(x_{0}, t_{0}\right)$, then $\mathcal{U}_{\delta, \xi}(x)<$ $u\left(x, t_{0}\right)$ in a small neighbourhood of $x_{0}$, while $\mathcal{U}_{6, \xi}(x)>u\left(x, t_{0}\right)$ for $x$ sufficiently far away from $x_{0}$; this would force the graphs of $\mathcal{U}_{\delta, \xi}$ and $u\left(\cdot, t_{0}\right)$ to have at least three intersections.

Finally, it follows from the tangency of the graphs of $\mathcal{U}_{\delta, \xi}$ and $u\left(\cdot, t_{0}\right)$ that $\mathcal{U}_{\delta, \xi}^{\prime \prime}\left(x_{0}\right) \geq$ $u_{x x}\left(x_{0}, t_{0}\right)$ implies

$$
\begin{aligned}
u_{t}\left(x_{0}, t_{0}\right) & =\frac{u_{x x}}{1+\left(u_{x}\right)^{2}}-\frac{n-1}{u} \\
& \leq \frac{\mathcal{U}_{\delta, \xi}^{\prime \prime}}{1+\left(\mathcal{U}_{\delta, \xi}^{\prime}\right)^{2}}-\frac{n-1}{\mathcal{U}_{\delta, \xi}} \\
& =0 .
\end{aligned}
$$

By differentiating the horizontal graph equation with respect to time, one obtains a linear parabolic PDE for $u_{t}$ (with smooth coefficients) for $a(t)<x<b(t), \tau<t<T$. An application of the maximum principle yields $u_{t}\left(x_{0}, t_{0}\right)<0$ whenever $u\left(x_{0}, t_{0}\right)<\varepsilon \rho$. Therefore the mean curvature at $r=u\left(x_{0}, t_{0}\right)$ satisfies

$$
H\left(x_{0}, t_{0}\right)=\left(\frac{-u_{t}}{\sqrt{1+u_{x}^{2}}}\right)_{\left(x_{0}, t_{0}\right)}>0 .
$$

Q. E. D.

4.6. Corollary. If $u\left(x_{0}, t_{0}\right) \leq \varepsilon \rho$, and $\delta, \xi$ are such that $\mathcal{U}_{\delta, \xi}\left(x_{0}\right)=u\left(x_{0}, t_{0}\right), \mathcal{U}_{\delta, \xi}^{\prime}\left(x_{0}\right)=$ $u_{x}\left(x_{0}, t_{0}\right)$, then $u\left(x, t_{0}\right) \leq \mathcal{U}_{\delta, \xi}(x)$ for all $x$ at which both sides of the inequality are defined.

In other words, if $u\left(x_{0}, t_{0}\right) \leq \varepsilon \rho$, then the graph $\gamma$ of $u\left(\cdot, t_{0}\right)$ lies below the graph of the (generalized) catenoid tangent to $\gamma$ at $\left(x_{0}, u\left(x_{0}, t_{0}\right)\right)$.

The corollary follows directly from the previous lemma: Since the graphs of $u\left(\cdot, t_{0}\right)$ and $\mathcal{U}_{\delta, \xi}$ are tangent, they have a double intersection, and they cannot have any other intersections. Thus either $u\left(x, t_{0}\right) \leq \mathcal{U}_{\delta, \xi}(x)$ for all $x$, or $u\left(x, t_{0}\right) \geq \mathcal{U}_{\delta, \xi}(x)$. The second situation does not occur since $u$ is bounded and $\mathcal{U}_{\delta, \xi}$ is not. 


\section{Altschuler, Angenent and Giga}

The necks of an evolving domain. Let $D \subset[0, \infty) \times \mathrm{R}^{n+1}$ be an inner evolution such that $\partial D(t)=\Gamma(t)$ is a smooth hypersurface for $0 \leq t<T$ of the form $r=u(x, t), a(t) \leq$ $x \leq b(t)$, for certain smooth functions $a, b:[0, T) \rightarrow \mathbf{R}$.

Let $k(t)$ be the number of local minima of $u(t, x)$ as a function of $x \in(a(t), b(t))$. Note that since $u$ vanishes at the endpoints of $(a(t), b(t))$, the number of local maxima is $k(t)+1$.

4.7. Lemma. $k(t)$ is a finite nonincreasing function of $t, 0 \leq t<T$.

Proof. Since $\Gamma(t)$ is a smooth hypersurface, we have

$$
\lim _{x \downarrow a(t)} u_{x}(x, t)=\infty, \quad \lim _{x \uparrow b(t)} u_{x}(x, t)=-\infty
$$

for $0<t<T$. As the boundary $\Gamma(t)$ evolves by mean curvature the function $u(x, t)$ is a solution of the horizontal graph equation on the domain

$$
\Omega=\{(x, t): a(t)<x<b(t), 0<t<T\} .
$$

By differentiating the horizontal graph equation with respect to $x$, one finds that $u_{x}$ solves the following linear parabolic equation

$$
v_{t}=\frac{v_{x x}}{1+\left(v_{x}\right)^{2}}+\left(\frac{n-1}{u^{2}}-2\left(\frac{u_{x x}}{1+\left(u_{x}\right)^{2}}\right)^{2}\right) v,
$$

on $\Omega$. Hence, by the Sturmian theorem the number of sign changes of $u_{x}$ is finite for any $t>0$, and does not increase. Since this number is $2 k(t)+1$, we are done.

Q. E. D.

\section{$\S 5$. Formation of a singularity.}

A final segment of a smooth evolution. In this section we consider a smooth classical solution $\Gamma(t), 0<t<T$ of the mean curvature flow, which becomes singular at $t=T$. We assume that $\Gamma(t)$ is rotationally symmetric, and that $\Gamma(t)$ can be represented as a horizontal graph $r=u(x, t), a(t)<x<b(t), 0<t<T$. As we showed in the previous section, the number of necks of $\Gamma(t)$ is a finite nonincreasing function of time. It follows that, after a while, the number of necks remains constant. After discarding an initial section of the solution we may even assume that each hypersurface $\Gamma(t)$ has $m \geq 0$ necks. In particular, $x \mapsto u(x, t)$ will have $m$ local minima and $m+1$ local maxima. Let these minima and maxima be located at $\left\{\xi_{j}(t)\right\}_{1 \leq j \leq m}$ and $\left\{\eta_{j}(t)\right\}_{0 \leq j \leq m}$ respectively; order the $\xi_{j}$ and $\eta_{j}$ so that

$$
a(t)<\eta_{0}(t)<\xi_{1}(t)<\eta_{1}(t)<\cdots<\xi_{m}(t)<\eta_{m}(t)<b(t) .
$$




\section{Mean Curvature Flow Through Singularities for Surfaces of Rotation.}

Since the number of critical points of $u(\cdot, t)$ drops whenever $u(\cdot, t)$ has a degenerate critical point, the minima and maxima of $u(\cdot, t)$ are all nondegenerate. By the implicit function theorem the $\xi_{j}(t)$ and $\eta_{j}(t)$ are therefore smooth functions of time.

\subsection{Converging Necks Lemma. The limits}

$$
\lim _{t \uparrow T} \xi_{j}(t)=\xi_{j}(T) \quad \text { and } \quad \lim _{t \uparrow T} \eta_{j}(t)=\eta_{j}(T)
$$

exist.

Not only do the necks converge as $t \uparrow T$, the end points $(a(t), 0)$ and $(b(t), 0)$ also converge to certain points $(a(T), 0)$ and $(b(T), 0)$ on the $x$-axis. This follows directly from the moving caps lemma which says that $a(t)$ and $b(t)$ are monotone increasing and decreasing functions of $t$, respectively. [CM].

The proof that the $\xi_{j}(t)$ and $\eta_{j}(t)$ converge uses a trick of Matano and X. Y. Chen

PROOF. Assume that $\xi_{j}(t)$ does not converge as $t \uparrow T$. Then $\liminf \inf _{t \uparrow T} \xi_{j}(t)<$ $\lim \sup _{t \uparrow T} \xi_{j}(t)$, and we can choose an $x_{1} \in\left(\liminf { }_{t \uparrow T} \xi_{j}(t), \lim \sup _{t \uparrow T} \xi_{j}(t)\right)$. Since $\xi_{j}(t)$ is continuous, there is an infinite sequence of times $t_{k} \uparrow T$ at which $\xi_{j}\left(t_{k}\right)=x_{1}$, and at which therefore $u_{x}\left(x_{1}, t_{k}\right)=0$ holds.

Consider the family of hypersurfaces $\tilde{\Gamma}(t)$ obtained by reflecting $\Gamma(t)$ in the hyperplane $x=x_{1}$. This family also evolves by its mean curvature, and the number of intersections of $\Gamma(t)$ with $\tilde{\Gamma}(t)$ is a finite nonincreasing function of time; moreover, this number drops whenever $\Gamma(t)$ and $\tilde{\Gamma}(t)$ intersect nontransversally. It follows that there is a $t_{*}<T$ such that $\Gamma(t)$ is transverse to $\tilde{\Gamma}(t)$ for $t_{*}<t<T$. But if $\Gamma(t)$ and $\tilde{\Gamma}(t)$ are transverse, then $u_{x}\left(x_{1}, t\right) \neq 0$; so we see that $u_{x}\left(x_{1}, t\right) \neq 0$ for $t_{*}<t<T$, which contradicts $u_{x}\left(x_{1}, t_{k}\right)=0$ for $t_{k} \uparrow T$. We must therefore conclude that $\xi_{j}(t)$ converges after all.

The same argument also shows that the $\eta_{j}(t)$ 's converge.

Q. E. D.

$X$. Y. Chen has also proved a stronger version of the following lemma; since the proof of our lemma is so simple we include it anyway.

5.2. Single Point Pinching Lemma. If $\eta_{j-1}(T)<\xi_{j}(T)$, then $u(x, t)$ is bounded from below uniformly in $t \in(0, T)$, for all $x \in\left(\eta_{j-1}(T), \xi_{j}(T)\right)$; i.e. for any compact interval $[c, d] \subset\left(\eta_{j-1}(T), \xi_{j}(T)\right)$ there exists a $\delta>0$ such that $u(x, t) \geq \delta$ for $x \in[c, d], t \in(0, T)$.

Similarly, if $\eta_{j}(T)>\xi_{j}(T)$ (or $a(T)<\eta_{0}(T)$, or $\eta_{m}(T)<b(T)$ ), then $u(x, t)$ is bounded from below, uniformly in $t$, for any $x \in\left(\xi_{j}(T), \eta_{j}(T)\right)$ (or $\left(a(T), \eta_{0}(T)\right)$, or $\left.\left(\eta_{0}(T), b(T)\right)\right)$.

ProOF. We prove the first case. Let $[a, b] \subset\left(\xi_{j}(T), \eta_{j}(T)\right)$ be any compact interval; then the converging necks lemma implies that there is a $t_{1}<T$ such that $u_{x}>0$ on $[a, b]$ for all $t_{1}<t<T$. Define $\theta=\arctan u_{x}(x, t)$. A simple calculation shows that $u(x, t)$ 


\section{Altschuler, Angenent and Giga}

satisfies $u_{t}=\theta_{x}-(n-1) / u$, and hence

$$
\theta_{t}-\frac{\theta_{x x}}{1+\left(u_{x}\right)^{2}}=\frac{n-1}{u^{2}} \sin \theta \cos \theta>0 .
$$

On the other hand, for any $\varepsilon>0$ the function

$$
\varphi(x, t)=\varepsilon e^{-\lambda^{2} t} \sin (\lambda(x-a)), \quad \lambda==_{\text {def }} \frac{\pi}{b-a},
$$

satisfies the heat equation $\varphi_{t}=\varphi_{x x}$; using $\varphi_{x x}<0$ on the interval $(a, b)$ one derives from this that

$$
\varphi_{t}-\frac{\varphi_{x x}}{1+\left(u_{x}\right)^{2}}=\frac{\left(u_{x}\right)^{2}}{1+\left(u_{x}\right)^{2}} \varphi_{x x}<0 .
$$

If one chooses $\varepsilon$ small enough, then $\varphi\left(x, t_{1}\right) \leq \theta\left(x, t_{1}\right)$ for all $x \in[a, b]$, and since $\varphi(x, t)$ vanishes when $x=a$ or $x=b$, we can apply the classical maximum principle to conclude that $\theta(x, t) \geq \varphi(x, t)$ for all $a<x<b, t_{1}<t<T$.

This implies that $u_{x}(x, t)=\tan \theta(x, t)$ is bounded from below by $\varepsilon e^{-\lambda^{2} T} \sin (\lambda(x-a))$, for all $t<T$ and $x \in(a, b)$; if we integrate this we find that

$$
u(x, t) \geq \frac{\varepsilon}{\lambda} e^{-\lambda^{2} T}(1-\cos \lambda(x-a)), \quad(a<x<b, 0<t<T) .
$$

Given the interval $[c, d] \subset\left(\eta_{j-1}(T), \xi_{j}(T)\right)$, we choose $[a, b] \subset\left(\eta_{j-1}(T), \xi_{j}(T)\right)$ to be a slightly larger interval. The lower bound for $u(x, t)$ on $(a, b)$ which we have just derived then implies the desired strict lower bound for $u$ on $[c, d]$.

Q. E. D.

In [DK], Dzuik and Kawohl prove that the surface will pinch at a single point. They assume various conditions on the symmetry and the mean curvature of the surface. In their situation, only one neck is forming.

From here on we extend $u$ to a function on $\mathrm{R} \times(0, T)$, by defining $u(x, t)=0$ if $x \leq a(t)$ or $x \geq b(t)$.

5.3. Limit Surface Lemma. $\lim _{t \uparrow T} u(x, t)=u(x, T)$ exists, and $u(x, t)$ converges uniformly to $u(x, T)$ as $t \uparrow T$. The function $u$ is smooth at $(x, t) \in \mathrm{R} \times(0, T]$ provided that $u(x, t)>0$.

Proof. We begin with the observation that $u(x, t)$ is uniformly bounded from below on any closed interval $[c, d] \subset\left(\xi_{j-1}(T), \xi_{j}(T)\right)$. This implies, by theorem 4.3 that $u_{x}$ is uniformly bounded on any compact subinterval, which in turn implies that all higher derivatives $\partial_{x}^{k} u(x, t)$ are bounded on any compact subinterval of $(c, d)$. In particular $u_{t}(x, t)$ is uniformly bounded on such intervals, so that $u(\cdot, t)$ converges uniformly on any such interval. 


\section{Mean Curvature Flow Through Singularities for Surfaces of Rotation.}

The same considerations apply to any compact interval $[c, d] \subset\left(a(T), \xi_{1}(T)\right)$, or $[c, d] \subset\left(\xi_{m}(T), b(T)\right)$, or, in case $\Gamma(t)$ has no necks at all, $[c, d] \subset(a(T), b(T))$.

The conclusion is that $u(x, t)$ converges pointwise for every $x$, except possibly at $a(T), b(T)$ or the $\xi_{j}(T)$ 's. The convergence is uniform on any compact interval which does not contain any of these points.

To complete the proof of the lemma, we observe that the functions $u(\cdot, t)$ with $T / 2<$ $t<T$ are equicontinuous. This, together with the pointwise convergence on a dense subset of $R$ implies that they converge uniformly as $t \uparrow T$.

To prove the equicontinuity, we let $\varepsilon>0$ be given, and we shall show that for some $\delta=\delta(\varepsilon)>0$ one has $\left|u\left(x_{1}, t\right)-u\left(x_{2}, t\right)\right|<\varepsilon$, whenever $\left|x_{1}-x_{2}\right|<\delta$.

To be definite we assume that $x_{1}<x_{2}$, and since $u$ vanishes outside $(a(t), b(t))$, we may also assume that the $x_{i}$ have been chosen in this interval.

Suppose that $\left|u\left(x_{1}, t\right)-u\left(x_{2}, t\right)\right| \geq \varepsilon$. Then either $u\left(x_{1}, t\right) \geq \varepsilon$, or $u\left(x_{2}, t\right) \geq \varepsilon$, or both; we shall assume the former.

It follows from theorem 4.3(b) that $\left|u_{x}\right|<\sigma(\varepsilon / 2, T / 2)$ whenever $u(x, t) \geq \varepsilon / 2$ and $T / 2<t<T$. Thus, if $u \geq \varepsilon / 2$ on $\left(x_{1}, x_{2}\right)$, then one has

$$
x_{2}-x_{1} \geq \frac{\varepsilon}{\sigma(\varepsilon / 2, T / 2)} .
$$

On the other hand, if $u(x, t)<\varepsilon / 2$ somewhere in the interval $\left(x_{1}, x_{2}\right)$, then there is a smallest $x_{3}>x_{1}$ at which $u\left(x_{3}, t\right) \leq \varepsilon / 2$. On the interval $\left(x_{1}, x_{3}\right)$ we then have $u \geq \varepsilon / 2$, so that

$$
x_{2}-x_{1} \geq x_{3}-x_{1} \geq \frac{\varepsilon-\varepsilon / 2}{\sigma(\varepsilon / 2, T / 2)}=\frac{\varepsilon}{2 \sigma(\varepsilon / 2, T / 2)} .
$$

Hence $\delta(\varepsilon)=\varepsilon / 2 \sigma(\varepsilon / 2, T / 2)$ is a modulus of continuity for $u(\cdot, t)$.

The second statement follows from a priori estimates for $u_{x}$ in theorem 4.3.b and hence (see [LUS]) for all higher derivatives of $u$ in the interior of the region $u>0$.

Q. E. D.

The next lemma is a refinement of the moving caps lemma. It will be used to study the caps of a smooth solution as it becomes singular.

5.4. Lemma. Suppose that $u\left(\eta_{0}(T), T\right)>0$. Then $a(T)<\eta_{0}(T)$, and there exist $\varepsilon, M>0$ such that $\varepsilon<a^{\prime}(t)<M$ for $0<t<T$.

Proof. Put $\delta=\inf _{0<t<T} u\left(\eta_{0}(t), t\right)>0$ and consider

$$
w\left(y_{1}, \ldots, y_{n}, t\right)=v\left(\sqrt{y_{1}^{2}+\cdots+y_{n}^{2}}, t\right) .
$$

This function is a solution of the full graph equation, $w_{t}=g^{i j}(\nabla w) \nabla_{i} \nabla_{j} w$, and it is defined for $|y|<\delta, 0<t<T$. Clearly $w$ is uniformly bounded, so the Evans-Spruck estimates 


\section{Altschuler, Angenent and Giga}

imply that $\nabla w$ as well as all higher space derivatives $\nabla^{j} w$ are uniformly bounded on the region $Q=\{(y, t):|y| \leq \delta, T / 2 \leq t<T\}$.

In particular $w_{t}$ will be bounded on $Q$, so that $w(y, t)$ converges uniformly in $|y| \leq \delta / 2$ as $t \uparrow T$; since all space derivatives of $w$ are bounded they must also converge uniformly. We shall denote the limit by $w(y, T)$.

Since $w(y, t)=v(|y|, t)$ this shows that $v(r, t) \rightarrow v(r, T)$ uniformly in $0 \leq r \leq \delta / 2$ as $t \uparrow T$. We have also shown that $v_{t}(r, t)$ is uniformly bounded for $0 \leq r \leq \delta / 2, T / 2<t<T$. In view of $a(t)=v(0, t)=w(0, \ldots, 0, t)$ this means that we have found an upper bound for $a^{\prime}(t)$.

From the fact that $v(r, t)$ satisfies the vertical graph equation, one derives by differentiation a linear parabolic PDE for $v_{r}(r, t)$ :

$$
\frac{\partial v_{r}}{\partial t}=\frac{\left(v_{r}\right)_{r r}}{1+v_{r}^{2}}+\left(\frac{n-1}{r}-\frac{2 v_{r} v_{r r}}{\left(1+v_{r}^{2}\right)^{2}}\right)\left(v_{r}\right)_{r}-\frac{n-1}{r^{2}} v_{r} .
$$

Using the maximum principle one then easily shows that $v_{r}(r, T)>0$ for $0<r<\delta / 2$. Hence $v(0, T)<v(\delta / 2, T)$, and $a(T)<\eta_{0}(T)$, as claimed.

Finally, to complete the proof we must find a lower bound for $a^{\prime}(t)$ as $t \uparrow T$. We shall obtain such a lower bound by first extending $w(y, t)$ to a smooth solution of the full graph equation on $Q_{\varepsilon}=\{(y, t):|y|<\delta / 2, T / 2<t<T+\varepsilon\}$ for some small $\varepsilon>0$; then we can apply the moving caps lemma to the resulting hypersurface $x=w(y, t)$ to bound $a^{\prime}(t)$ from below.

Perhaps the simplest way to construct an extension of $w$ to $Q_{\varepsilon}$ is to let $\varphi$ be the solution of the initial-boundary value problem

$$
\begin{cases}\varphi_{t}=g^{i j}(\nabla \varphi) \varphi_{y_{i} y_{j}} & \text { for }|y|<\delta / 2, T<t<T+\varepsilon \\ \varphi(y, t)=w(y, T) & \text { when }|y|=\delta / 2 \\ \varphi(y, T)=w(y, T) & \text { when }|y|<\delta / 2\end{cases}
$$

In other words, we try to solve the full graph equation while prescribing $w(\cdot, T)$ as initial data, and while keeping the boundary values of $\varphi$ constant. Since the full graph equation is quasilinear, and since the initial and boundary values are $C^{\infty}$, a solution exists if $\varepsilon>0$ is sufficiently small. This solution will be $C^{\infty}$ up to its initial value at $t=T$, so we can extend $w$ by defining $w(y, t)=\varphi(y, t)$ for $t>T$.

Denote the ensuing family of hypersurfaces, defined by $x=w(y, t)$ for $(y, t) \in Q_{e}$, by $\Gamma^{*}(t)$.

Uniqueness of the solution implies that $w$ is rotationally symmetric, i.e. of the form $w(y, t)=v(|y|, t)$ where $v$ satisfies the vertical graph equation. The prescribed boundary values for $\varphi$ are constant, so the maximum principle implies that $w(y, t) \leq v(\delta / 2, T)$ for $|y|<\delta / 2, T<t<T+\varepsilon$. This in turn implies that $v_{r}(\delta / 2, t) \geq 0$ for $T<t<T+\varepsilon$, and by applying the maximum principle to the equation for $v_{r}$ we find that $v_{r}>0$ for $0<r<\delta / 2,0<t<T+\varepsilon$ (we already knew this for $t \leq T$.) 


\section{Mean Curvature Flow Through Singularities for Surfaces of Rotation.}

Thus we may apply the arguments in the moving caps lemma to the family of hypersurfaces $\Gamma^{*}(t)$, and conclude that $w_{t}(0, t)>0$ for $0<t<T+\varepsilon$. Since $w_{t}$ is continuous it follows that $w_{t}(0, t)$ and hence $a^{\prime}(t)$ are bounded from below for $0<t \leq T$.

Q. E. D.

Domains without necks. The lemmas which we have developed so far in this section can be illustrated by considering a family of hypersurfaces $\Gamma(t)$ without necks, i.e. for which $m=0$. Such a family may be represented by a horizontal graph $r=u(x, t), a(t)<x<$ $b(t), 0<t<T$, where $u(\cdot, t)$ attains a unique local maximum at $x=\eta_{0}(t)$. In general the domains enclosed by $\Gamma(t)$ need not be convex, but they are always starshaped with respect to the point $\left(\eta_{0}(t), 0\right)$.

Figure 5.1-A domain without necks.

Assume that that the $\Gamma(t)$ evolve by their mean curvature, and that they form a maximal classical solution.

If $\Gamma_{t_{0}}$ is convex for some $t_{0}$ then Huisken's by now classical result says that the $\Gamma(t)$ remain convex for $t \geq t_{0}$, and that they shrink to a "round point," i.e. they shrink to a point, and their asymptotic shape is a sphere.

It seems reasonable to conjecture that a family of surfaces "without necks" will eventually become convex and shrink to a round point, by Huisken's theorem. We don't have a proof of this, but we can show the following.

5.5. Lemma. Let $\{\Gamma(t): 0<t<T\}$ be a maximal classical solution of mean curvature flow without necks. Then $\Gamma(t)$ shrinks to a point as $t \uparrow T$.

Proof. We shall show that $u\left(\eta_{0}(T), T\right)=0$, and that $a(T)=b(T)$.

If $u\left(\eta_{0}(T), T\right)>0$, then lemma 5.4 would imply $a(T)<\eta_{0}(T)<b(T)$. Moreover, the left and right caps of $\Gamma(t)$ would remain uniformly smooth as $t \uparrow T$, as would the middle part of $\Gamma(t)$, i.e. the part contained in $a(T)+\varepsilon<x<b(T)-\varepsilon$, for any $\varepsilon>0$. Thus the curvatures of $\Gamma(t)$ would remain uniformly bounded as $t \uparrow T$, which is impossible, since $\Gamma(t)$ becomes singular at $t=T$.

If $a(T)<b(T)$, then either $a(T)<\eta_{0}(T)$ or $\eta_{0}(T)<b(T)$. In both cases the single point pinching lemma would imply that $u\left(\eta_{0}(T), T\right)>0$. But we have just seen that this cannot be true, so we must conclude that $a(T)=b(T)$.

Q. E. D.

A necessary condition for formation of a singularity. The arguments above allow one to prove the following lemma. 


\section{Altschuler, Angenent and Giga}

5.6. Lemma. If $\{\Gamma(t): 0<t<T\}$ is a maximal smooth solution of the mean curvature flow, which for $T-\varepsilon<t<T$ has $m \geq 1$ necks, for some $\varepsilon>0$, then there is a $1 \leq j \leq m$ such that $u\left(\xi_{j}(T), T\right)=0$.

In other words, unless a solution eventually loses its necks, it can only become singular if one of its necks shrinks to the axis. In particular, if the inside of $\Gamma(t)$ remains a $\delta$-domain, for some $\delta>0$ and for all $0<t<T$, then the solution extends to a smooth solution on a larger time interval.

Asymptotics of a shrinking neck. We continue our investigation of the maximal classical solution $\Gamma(t)$ given by $r=u(x, t)$. As before we assume that $\Gamma(t)$ has $m \geq 0$ necks, located at $x=\xi_{j}(t)$.

Let

$$
\rho_{j}(t)=u\left(\xi_{j}(t), t\right), \quad(j=1, \ldots, m)
$$

denote the radius of the $j^{\text {th }}$ neck. Since $u(\cdot, t)$ attains a local minimum at $x=\xi_{j}(t)$, we have $u_{x}=0, u_{x x} \geq 0$ at the neck, and hence, by the horizontal graph equation,

$$
\rho_{j}^{\prime}(t)=u_{t}\left(\xi_{j}(t), t\right) \geq-\frac{n-1}{\rho_{j}(t)} .
$$

If $\rho_{j}(T)=0$, then integration of this inequality shows that

$$
\rho_{j}(t) \leq \sqrt{2(n-1)(T-t)}
$$

The example of the shrinking cylinder shows that this estimate is in general optimal. It turns out that the reverse inequality is also true, up to a constant factor.

5.7. Contraction Rate Theorem. There exist constants $\rho, \delta>0$, depending on the family of hypersurfaces $\{\Gamma(t): 0<t<T\}$, such that

$$
\rho_{j}(t) \geq \sqrt{2 \delta(n-1)(T-t)}
$$

holds whenever $\rho_{j}(t)<\rho$ and $\rho_{j}(T)=0$.

We shall prove this theorem by adapting the arguments of Huisken in [H1, H2]. As Huisken does in that paper, we shall make essential use of the fact that the quantity $Z=|\mathrm{A} / H|^{2}$ satisfies a nice parabolic differential equation, for any smooth family of hypersurfaces $\{\Gamma(t): 0<t<T\}$ evolving by its mean curvature. Here $\mathrm{A}$ denotes the second fundamental form of the surface $\Gamma(t)$.

In terms of the principal curvatures $\kappa_{1}, \ldots, \kappa_{n}$ of the hypersurface, the quantity $Z$ can be written as

$$
Z=\frac{\kappa_{1}^{2}+\cdots+\kappa_{n}^{2}}{\left(\kappa_{1}+\cdots+\kappa_{n}\right)^{2}} .
$$




\section{Mean Curvature Flow Through Singularities for Surfaces of Rotation.}

The equation it satisfies is

$$
\frac{\partial Z}{\partial t}-\Delta_{\Gamma(t)} Z-B \cdot \nabla Z=-2\left|\nabla \frac{\mathbf{A}}{H}\right|^{2} \leq 0,
$$

with $\Delta_{\Gamma(t)}$ the Laplace-Beltrami operator on the hypersurface $\Gamma(t)$, and $B$ the vector field $\nabla\left(\log H^{2}\right)$. This equation is valid wherever $H \neq 0$, i.e. wherever $Z$ is well defined.

The time derivative is taken with respect to a normal parametrization of the family $\Gamma(t)$ : i.e. one parametrizes a neighborhood of a point $(P, t) \in \Gamma(t) \times\{t\} \subset \mathbf{R}^{n+1} \times[0, \infty)$ by a family of immersions $X: \mathcal{O} \times\left(t_{0}, t_{1}\right) \rightarrow \mathrm{R}^{n+1}$ for some open subset $\mathcal{O} \subset \mathrm{R}^{n}$, and one arranges that the immersions $X(\cdot, t)$ evolve via

$$
\frac{\partial X}{\partial t}=H \cdot N
$$

where $\mathrm{N}$ is a unit normal vector field to $\Gamma(t)$. The differential geometric quantities $\mathrm{A}, H$ and $Z$ then become functions on $\mathcal{O} \times\left(t_{0}, t_{1}\right)$ which is where the equation (5.2) must be interpreted. We refer to [H1, lemma 5.2] for more details, and a derivation of this equation.

The principal use of (5.2) is that it implies that $Z$ does not attain an interior maximum, due to the maximum principle. If $H \neq 0$ on all of $\Gamma(t)$, then this leads to an upper bound for $Z$, which in turn allows one to estimate all the principal curvatures in terms of the mean curvature.

Since we are not assuming that $H>0$ on all of $\Gamma(t), Z$ is not necessarily defined everywhere, and we cannot bound $Z$ as easily. Fortunately we are only interested in the behaviour of $\Gamma(t)$ near the axis, where the attracting axis lemma guarantees that $H>0$.

Let $\rho>0$ be so small that $H>0$ at any point on $\Gamma(t)$ with $r<2 \rho$, and define $\Sigma_{t} \subset \Gamma(t)$ to consist of all points with $r \leq \rho$. Then we have the following.

\subsection{Lemma. For any $t_{0}>0, Z$ is uniformly bounded on $\Sigma_{t}$, for $t_{0} \leq t<T$.}

Proof. By the maximum principle it will suffice to show that $Z$ is uniformly bounded on $\Sigma_{t_{0}}$, and on $\partial \Sigma_{t}$ for $t_{0} \leq t<T$.

The estimate on $\Sigma_{t_{0}}$ is easy: $\Sigma_{t_{0}}$ is smooth and $H$ is bounded away from zero on $\Sigma_{t_{0}}$, so $Z=|\mathrm{A} / H|^{2}$ is certainly bounded on $\Sigma_{t_{0}}$.

The boundaries $\partial \Sigma_{t}$ consist of all points $(x, y) \in \mathrm{R} \times \mathrm{R}^{n}$ with $|y|=u(x, t)=\rho$. Let

$$
S=\left\{(x, t) \in \mathrm{R} \times\left[t_{0}, T\right]: u(x, t)=\rho\right\} .
$$

Then $S$ is closed in $\mathrm{R} \times\left[t_{0}, T\right]$, and there is an $\varepsilon$ neighborhood of $S$, which we shall denote by $S_{\varepsilon}$, on which $u(x, t) \geq \rho / 2$. On $S_{\varepsilon} u$ will be a smooth solution of the horizontal graph equation, whose derivatives are uniformly bounded on the smaller neighborhood $S_{\varepsilon / 2}$ of $S$.

By differentiating the horizontal graph equation with respect to time, one obtains a linear parabolic PDE for $u_{t}$, on $S_{\epsilon / 2}$. The coefficients in this equation are smooth on $S_{\varepsilon / 2}$, so one can apply the maximum principle, and conclude that $u_{t}$, and therefore

$$
H=\frac{-u_{t}}{\sqrt{1+u_{x}^{2}}}
$$




\section{Altschuler, Angenent and Giga}

are bounded away from zero on $S_{\varepsilon / 2}$. Consequently we have found that $Z$ is indeed uniformly bounded on the $\partial \Sigma_{t}$ 's.

Q. E. D.

Proof of the Contraction Rate Theorem. Choose $C<\infty$ so that $Z \leq C^{2}$ whenever $r \leq \rho$. Then from

$$
|A|^{2}=\frac{u_{x x}^{2}}{\left(1+u_{x}^{2}\right)^{3}}+\frac{n-1}{u^{2}\left(1+u_{x}^{2}\right)}
$$

we find that

$$
\frac{u_{x x}^{2}}{\left(1+u_{x}^{2}\right)^{3}} \leq|\mathbf{A}|^{2} \leq C^{2} H^{2}=C^{2}\left(\frac{u_{x x}}{\left(1+u_{x}^{2}\right)^{3 / 2}}-\frac{n-1}{u\left(1+u_{x}^{2}\right)^{1 / 2}}\right)^{2} .
$$

Using $H>0$ on $\Sigma_{t}$ we get, after multiplying both sides with $1+u_{x}^{2}$ and extracting square roots,

$$
\begin{aligned}
\frac{u_{x x}}{1+u_{x}^{2}} & \leq C\left(\frac{n-1}{u}-\frac{u_{x x}}{1+u_{x}^{2}}\right) \\
& \leq \frac{C}{C+1} \frac{n-1}{u} .
\end{aligned}
$$

Hence, if we define $\delta=(1+C)^{-1}$,

$$
\frac{\partial u}{\partial t}=\frac{u_{x x}}{1+u_{x}^{2}}-\frac{n-1}{u} \leq-\frac{\delta(n-1)}{u} .
$$

For the width of the $j^{\text {th }}$ neck, $\rho_{j}(t)$ we therefore find that

$$
\rho_{j}^{\prime}(t) \leq-\frac{\delta(n-1)}{\rho_{j}(t)}
$$

which, after integration leads to the desired estimate.

Q. E. D.

One can use the inequality (5.3) to prove something a little more general, namely

$$
u(x, t) \geq \sqrt{u(x, T)^{2}+2 \delta(n-1)(T-t)}
$$

which holds as long as the right hand side does not exceed $\rho$.

We have found no application for this more general estimate, but one could use it to turn a lower bound for $u(x, T)$ near $x=\xi_{j}(T)$ into an estimate for the rate at which $\xi_{j}(t)$ converges to $\xi_{j}(T)$. E.g. X. Y. Chen has proved that there exist constants $C_{\varepsilon}<\infty$ such that

$$
u(x, T) \geq C_{\varepsilon}\left|x-\xi_{j}(T)\right|^{1+\varepsilon}
$$

for any $\varepsilon>0$. Hence $u\left(\xi_{j}(t), T\right)^{2} \leq u\left(\xi_{j}(t), t\right)^{2}-2 \delta(n-1)(T-t) \leq 2(1-\delta)(n-1)(T-t)$, and we see that $X$. Y. Chen's estimate implies

$$
\left|\xi_{j}(t)-\xi_{j}(T)\right| \leq C_{\varepsilon}^{\prime}(T-t)^{1-\varepsilon}
$$

for any $\varepsilon>0$. 
Mean Curvature Flow Through Singularities for Surfaces of Rotation.

5.9. Cylindrical Blow-up Theorem. If the $j^{\text {th }}$ neck pinches at $t=T$, i.e. if $\rho_{j}(T)=0$, and if $a(T)<\xi_{j}(T)<b(T)$, then

$$
\lim _{t \uparrow T} \frac{u\left(\xi_{j}(T)+z \sqrt{2(T-t)}, t\right)}{\sqrt{2(T-t)}}=\sqrt{n-1} .
$$

The convergence is uniform in $z$ on any bounded interval $|z| \leq M$.

This type of asymptotics of blow-up was first proved in [GK] for semilinear heat equations. For $n=2$ and under the assumption that $H \geq 0$, Huisken [H2] obtained the same asymptotic formula. The proof in [H2] does not seem to generalize to the higher dimensional case.

The following corollary of this blow-up result will be crucial for our line of reasoning in section 7. It is a pleasure to thank Tom Ilmanen for telling us about this nice observation.

5.10. Corollary (Ilmanen). The singular necks of the limit surface are fiat, i.e. if $\rho_{j}(T)=0$, and $a(T)<\xi_{j}(T)<b(T)$, then for any $\varepsilon>0$ there is a $\mu_{\varepsilon}>0$ such that

$$
u(x, T) \leq \varepsilon\left|x-\xi_{j}(T)\right|
$$

for $\left|x-\xi_{j}(T)\right| \leq \mu_{\varepsilon}$.

Proof of THE COROLlary. Given $\varepsilon>0$ put $M=2 \sqrt{n-1} / \varepsilon$. By the cylindrical blow-up theorem there is a $t_{e}<T$ for which

$$
u\left(\xi_{j}(t)+z \sqrt{2(T-t)}, t\right) \leq 2 \sqrt{2(n-1)(T-t)}
$$

for $|z| \leq M$ and $t_{\varepsilon}<t<T$. We may assume that $t_{\varepsilon}$ is so close to $T$ that

$$
2 \sqrt{2(n-1)\left(T-t_{\varepsilon}\right)}<\rho,
$$

so that $u_{t} \leq 0$ for $|z| \leq M$ and $t_{\varepsilon}<t<T$, by the attracting axis lemma.

Let $\mu_{\varepsilon}=M\left(2\left(T-t_{\varepsilon}\right)\right)^{1 / 2}$. Then any $x \neq \xi_{j}(T)$ with $\left|x-\xi_{j}(T)\right| \leq \mu_{\varepsilon}$ can be written as $x=\xi_{j}(T) \pm M(2(T-t))^{1 / 2}$, for some $t \in\left(t_{e}, T\right)$. Using $u_{t} \leq 0$ and (5.4) we therefore find that

$$
\begin{aligned}
u(x, T) & \leq u(x, t) \leq 2 \sqrt{2(n-1)(T-t)} \\
& \leq 2 \sqrt{n-1}\left|\frac{x-\xi_{j}(T)}{M}\right| \\
& \leq \varepsilon\left|x-\xi_{j}(T)\right|,
\end{aligned}
$$

as claimed. The inequality is obviously true when $x=\xi_{j}(T)$.

Q.E. D. 


\section{Altschuler, Angenent and Giga}

Before we prove the blow-up theorem we must recall Huisken's Monotonicity Formula since finding a Lyapunov function will play a prominent role in our proof.

Consider the new time variable $\tau$, which is related to $t$ via

$$
\tau=-\log \sqrt{T-t}, \quad t=T-e^{-2 \tau}
$$

For each $\tau>-\log \sqrt{T}$ we let $\hat{\Gamma}(\tau)$ be the hypersurface obtained by magnifying the surface $\Gamma(t)$ by a factor $(2(T-t))^{-1 / 2}$, and translating it along the $x$-axis so that the point $\left(\xi_{j}(T), 0\right)$ gets moved to the origin: i.e.

$$
\hat{\Gamma}(\tau)=\Phi_{t}(\Gamma(t)), \quad \text { where } \quad \Phi_{t}(x)=\frac{x-P_{j}}{\sqrt{2(T-t)}}
$$

and $P_{j}=\left(\xi_{j}(T), 0, \ldots, 0\right)$.

The normalized surfaces $\hat{\Gamma}(\tau)$ move by their mean curvature "plus a lower order term." Their normal velocity $V$ at a point $Y \in \hat{\Gamma}(\tau)$ is given by

$$
V=\hat{H}+(Y, N)
$$

with $\mathrm{N}$ the unit normal to $\hat{\Gamma}(\tau)$ at $Y$, and $\hat{H}$ the mean curvature of $\hat{\Gamma}(\tau)$ at $Y$.

If $\Gamma(t)$ is given by the horizontal graph $r=u(x, t),(a(t)<x<b(t))$, then $\hat{\Gamma}(\tau)$ is also given by a horizontal graph, $r=U(z, \tau)$, where

$$
U(z, \tau)=\frac{u\left(\xi_{j}(t)+z \sqrt{2(T-t)}, t\right)}{\sqrt{2(T-t)}}
$$

A short computation reveals that $U(z, \tau)$ satisfies

$$
\frac{\partial U}{\partial \tau}=\frac{U_{z z}}{1+U_{z}^{2}}-z U_{z}+U-\frac{n-1}{U}
$$

for $A(\tau)<z<B(\tau)$, where

$$
A(\tau)=\frac{a(t)-\xi_{j}(T)}{\sqrt{2(T-t)}}, \quad B(\tau)=\frac{b(t)-\xi_{j}(T)}{\sqrt{2(T-t)}} .
$$

Our assumption $a(T)<\xi_{j}(T)<b(T)$ implies that $B(\tau) \rightarrow \infty$ and $A(\tau) \rightarrow-\infty$ as $\tau \rightarrow \infty$, so that $U(\cdot, \tau)$ is defined on an ever increasing interval.

In terms of the rescaled surfaces $\hat{\Gamma}(\tau)$ Huisken's monotonicity formula [H2] may be stated as follows:

$$
\frac{d}{d \tau} \int_{\hat{\Gamma}(\tau)} e^{-|Y|^{2} / 2} d \mathcal{H}^{n}=-\int_{\hat{\Gamma}(\tau)} e^{-|Y|^{2} / 2}(\hat{H}+(Y, N))^{2} d \mathcal{H}^{n}
$$




\section{Mean Curvature Flow Through Singularities for Surfaces of Rotation.}

where $d \mathfrak{H}^{n}$ denotes the $n$-dimensional Hausdorff measure on $\hat{\Gamma}(\tau)$.

If we denote the graph of $U(\cdot, \tau)$ by $\gamma(\tau)$ (so that $\gamma(\tau)$ is a curve in the upper half plane whose endpoints are on the $x$-axis), then the monotonicity formula (5.6) may be written as

$$
\frac{d}{d \tau} \int_{\gamma(\tau)} e^{-\left(x^{2}+r^{2}\right) / 2} r^{n-1} d s=-\int_{\gamma(\tau)} e^{-\left(x^{2}+r^{2}\right) / 2} r^{n-1}(k+K(x, r, \theta))^{2} d s .
$$

Here $d s$ denotes Euclidean arc length along $\gamma(\tau)$,

$$
k=\frac{-U_{z z}}{\left(1+U_{z}^{2}\right)^{3 / 2}}, \quad \theta=\arctan U_{z}
$$

are the curvature and tangent-angle of $\gamma(\tau)$, and the function $K: \mathrm{R} \times \mathrm{R}^{+} \times \mathrm{R} / 2 \pi \mathrm{Z} \rightarrow \mathrm{R}$ is given by

$$
K(x, r, \theta)=-\left(r-\frac{n-1}{r}\right) \cos \theta+x \sin \theta .
$$

The relevance of this function for our discussion lies in the following: If $U(z, \tau)$ satisfies (5.5), then its graph $\gamma(\tau)$ evolves with normal velocity $k+K(x, r, \theta)$.

Proof of the blow-up theorem. The monotonicity formula shows that the length of $\gamma(\tau)$ in the metric

$$
\chi_{n}=(d s)^{2}=e^{-\left(x^{2}+r^{2}\right) r^{2(n-1)}}\left((d x)^{2}+(d r)^{2}\right)
$$

on the upper half plane is nonincreasing. If the family of curves $\{\gamma(\tau)\}$ is compact, in some sense, then the monotonicity formula also suggests that the limit $\gamma_{*}$ of any convergent sequence $\gamma\left(\tau_{k}\right)$ will be a geodesic of the metric $\chi_{n}$, i.e. it will satisfy

$$
k+K(x, r, \theta)=0 \text {. }
$$

Equivalently, $\gamma_{*}$ will be the graph of some function $U_{*}$ on some open subset of $R$, which satisfies

$$
\frac{U_{*}^{\prime \prime}(z)}{1+U_{*}^{\prime}(z)^{2}}-z U_{*}^{\prime}(z)+U_{*}(z)-\frac{n-1}{U_{*}(z)}=0 .
$$

G. Huisken [H3] has shown that the only possible solutions of this equation, with $H \geq 0$ are the "cylindric solution" $U_{*}(z) \equiv \sqrt{n-1}$, and the "spherical solution" $U(z)=\sqrt{n-z^{2}}$."

Below we shall show that one can indeed extract convergent subsequences from $\gamma(\tau)$, and that they all converge to the cylindric solution $U_{*}(z) \equiv \sqrt{n-1}$. We shall conclude from this that the whole family $\gamma(\tau)$ converges to the straight line $r=\sqrt{n-1}$.

There are two ways in which a sequence $\gamma\left(\tau_{k}\right)$ conceivably could fail to have a convergent subsequence: The sequence could loose regularity, i.e. its curvature could blow up, or the sequence could wander off to infinity, so that for any $R>0$ the sequence $\gamma\left(\tau_{k}\right)$ would eventually lie outside the region $|z|<R, r<R$. The following two lemmas show that neither of these two situations can occur. 


\section{Altschuler, Angenent and Giga}

5.11. Lemma. For any $t<T$ the surface $\Gamma(t)$ intersects the ball $\bar{B}\left(P_{j}, \sqrt{2 n(T-t)}\right)$, and the surface $\hat{\Gamma}(\tau)$ intersects the ball $\bar{B}(0, \sqrt{n})$. Here $P_{j}$ denotes the point $\left(\xi_{j}(T), 0\right)$.

Proof. The two statements are equivalent, so we only have to prove the first one.

Suppose that for some $t_{1}<T$ the closed ball $\bar{B}\left(P_{j},\left(2 n\left(T-t_{1}\right)\right)^{1 / 2}\right)$ and the surface $\Gamma\left(t_{1}\right)$ are disjoint. Since $\Gamma\left(t_{1}\right)$ is compact, $\Gamma\left(t_{1}\right)$ will also be disjoint from a slightly larger ball. We shall write the radius of this ball as $\left(2 n\left(T^{\prime}-t_{1}\right)\right)^{1 / 2}$, where $T^{\prime}$ is slightly larger than $T$.

By the separation theorem it follows that $\Gamma(T)$ and $\bar{B}\left(P_{j},\left(2 n\left(T^{\prime}-T\right)\right)^{1 / 2}\right)$ will be disjoint; but this is not possible, since both $\bar{B}\left(P_{j},\left(2 n\left(T^{\prime}-T\right)\right)^{1 / 2}\right)$ and $\Gamma(T)$ contain $P_{j}$. Q. E. D.

5.12. Lemma. For any $R>0$ there exist $\tau_{R}, M_{R}<\infty$ such that $|k| \leq M_{R}$ at any point $(z, r)$ on $\gamma(\tau)$ with $|z| \leq R, r \leq R$ and $\tau \geq \tau_{R}$.

The proof will consist of two steps. In the first we establish a bound for $U_{z}$ in the relevant region; in the second we show how one can use interior estimates for the renormalized graph equation (5.5) to bound the curvature of $\gamma(\tau)$.

Let $\rho>0$ be as in the attracting axis lemma, i.e. if $u(x, t) \leq \rho$, then $u_{t}(x, t)<0$.

5.13. Lemma. If $u\left(x_{0}, t_{0}\right) \leq \rho$, and $\eta_{0}\left(t_{0}\right) \leq x_{0} \leq \eta_{m}\left(t_{0}\right)$, then

$$
\left|u_{x}\left(x_{0}, t_{0}\right)\right| \leq\left(\frac{u\left(x_{0}, t_{0}\right)}{\sqrt{2 \delta(n-1)\left(T-t_{0}\right)}}\right)^{n-1} .
$$

Proof. Assume for definiteness that $u_{x}\left(x_{0}, t_{0}\right)>0$. Then $u\left(\cdot, t_{0}\right)$ must attain a local minimum between $\eta_{0}\left(t_{0}\right)$ and $x_{0}$. Let $\xi_{i}\left(t_{0}\right)$ be the largest $\xi_{k}\left(t_{0}\right)<x_{0}$.

Since. $u\left(x_{0}, t_{0}\right) \leq \rho$ we have $u\left(x, t_{0}\right) \leq \rho$ for $\xi_{i}\left(t_{0}\right) \leq x \leq x_{0}$, and therefore, by the attracting axis lemma $u_{t}\left(x, t_{0}\right) \leq 0$, i.e.

$$
\frac{u_{x x}}{1+u_{x}^{2}} \leq \frac{n-1}{u}
$$

After multiplying both sides with $u_{x}$ and integrating from $\xi_{i}\left(t_{0}\right)$ to $x_{0}$ one obtains

$$
\left(u_{x}\left(x_{0}, t_{0}\right)\right)^{2} \leq\left(\frac{u\left(x_{0}, t_{0}\right)}{u\left(\xi_{i}\left(t_{0}\right), t_{0}\right)}\right)^{2(n-1)}-1 .
$$

The desired inequality now follows from the contraction rate theorem which gives a bound on $u\left(\xi_{i}\left(t_{0}\right), t_{0}\right)$ from below by $\sqrt{2 \delta(n-1)(T-t)}$.

Q. E. D. 
Mean Curvature Flow Through Singularities for Surfaces of Rotation.

After rescaling, this proposition has the following implication for $U_{z}(z, \tau)$ :

$$
\left|U_{z}(z, \tau)\right| \leq\left(\frac{U(z, \tau)}{\sqrt{\delta(n-1)}}\right)^{n-1},
$$

whenever $U(z, \tau) \leq \rho e^{\tau}$ and $\hat{\eta}_{0}(\tau) \leq z \leq \hat{\eta}_{m}(\tau)$, where by definition

$$
\hat{\eta}_{k}(\tau)=\frac{\eta_{k}(t)-\xi_{j}(T)}{\sqrt{T-t}}
$$

It follows from the single point pinching lemma that our assumption $a(T)<\xi_{j}(T)<b(T)$ implies $\eta_{0}(T)<\xi_{j}(T)<\eta_{m}(T)$, and thus that $\hat{\eta}_{0}(\tau) \rightarrow-\infty$ and $\hat{\eta}_{m}(\tau) \rightarrow \infty$ as $\tau \rightarrow \infty$.

Choose $\tau_{R}$ so large that

$$
\begin{gathered}
e^{\tau_{R}-1} \geq 2 R, \\
\hat{\eta}_{0}(\tau) \leq-2 R, \text { and } \hat{\eta}_{m}(\tau) \geq 2 R \text { for } \tau \geq \tau_{R}-1 .
\end{gathered}
$$

Then we have just shown that

$$
\left|U_{z}\right| \leq(R / \sqrt{\delta(n-1)})^{n-1}=\text { def } L_{R}
$$

whenever $U \leq R,|z| \leq 2 R$ and $\tau \geq \tau_{R}-1$. This is the estimate for $U_{z}$ which we said we would find. Next, we turn to the curvature of $\gamma(\tau)$.

As $U$ satisfies the renormalized graph equation, the bound for $\left|U_{z}\right|$ implies interior estimates for all higher derivatives of $U$. Thus to estimate, say, $U_{z z}$ at some point $\left(z_{0}, \tau_{0}\right)$ we must find a neighborhood of this point on which $U$ is defined and bounded by $2 R$. The interior estimates for quasilinear parabolic PDEs (see (LUS]) then give us a bound for $U_{z z}\left(z_{0}, \tau_{0}\right)$, and hence for the curvature of $\gamma\left(\tau_{0}\right)$ at the point $\left(z_{0}, U\left(z_{0}, \tau_{0}\right)\right)$.

With this motivation in mind we set out to prove the next lemma.

5.14. Lemma. Assume that $U\left(z_{0}, \tau_{0}\right) \leq R$ and $\left|z_{0}\right| \leq R, \tau \geq \tau_{R}$. Then there exist $\varepsilon=\varepsilon(\delta, R)>0$ and $\zeta=\zeta(\delta, R)>0$ such that $U(z, \tau) \leq 2 R$ for $\left|z-z_{0}\right| \leq \zeta$ and $\tau_{0}-\varepsilon<\tau<\tau_{0}$.

Furthermore, there exist constants $C_{j, R}<\infty(j \geq 1)$, such that $\left|\partial_{z}^{j} U\left(z_{0}, \tau_{0}\right)\right| \leq C_{j, R}$.

Proor. As we explained above, the second part of the lemma follows from the bound $\sqrt{\delta(n-1)} \leq U \leq 2 R$ in conjunction with the estimate for $\left|U_{z}\right|$ and the classical results in [LUS]. Thus we only have to verify the first statement.

We claim that there is an $M<\infty$ such that $\gamma(\tau)$ intersects the disk with radius $\sqrt{M\left(\tau_{0}-\tau\right)}$ centered at $\left(z_{0}, U\left(z_{0}, \tau_{0}\right)\right)$, for all $\tau \in\left(\tau_{0}-1, \tau_{0}\right)$. Indeed, $\left(z_{0}, U\left(z_{0}, \tau_{0}\right)\right)$ lies on $\gamma\left(\tau_{0}\right)$, so $\Gamma\left(t_{0}\right)$ contains the point

$$
P=\left(\xi_{j}+\sqrt{2\left(T-t_{0}\right)} z_{0}, \sqrt{2\left(T-t_{0}\right)} U\left(z_{0}, \tau_{0}\right) \mathrm{e}\right)
$$




\section{Altschuler, Angenent and Giga}

where $e \in R^{n}$ is any unit vector. By the separation theorem $\Gamma(t)$ must intersect the closed ball centered at $P$, with radius $\sqrt{2 n\left(t_{0}-t\right)}$, for all $t \leq t_{0}$; hence $\gamma(\tau)$ must intersect the disk determined by

$$
\begin{aligned}
\left(z-z_{0}\right)^{2}+\left(r-U\left(z_{0}, \tau_{0}\right)\right)^{2} & \leq 2 n \frac{t_{0}-t}{2\left(T-t_{0}\right)} \\
& =n\left(e^{2\left(\tau_{0}-\tau\right)}-1\right) \\
& \leq e^{2} n\left(\tau_{0}-\tau\right),
\end{aligned}
$$

as long as $\tau_{0}-1<\tau \leq \tau_{0}$. From this we see that we may even take $M=e^{2} n$.

Thus for each $\tau \in\left(\tau_{0}-\varepsilon, \tau_{0}\right)$ there is a $z_{\tau}$ with $\left|z_{\tau}-z_{0}\right| \leq \sqrt{M\left(\tau_{0}-\tau\right)} \leq \sqrt{M \varepsilon}$ such that

$$
U\left(z_{\tau}, \tau\right) \leq U\left(z_{0}, \tau_{0}\right)+\sqrt{M \varepsilon} \leq \frac{3 R}{2}
$$

provided $\varepsilon$ is chosen small enough $\left(\varepsilon<R^{2} / 4 M\right)$.

Since $\left|U_{z}\right| \leq L_{R}$ as long as $U \leq 2 R$, we have $U(z, \tau) \leq 2 R$ for all $(z, \tau)$ with $\left|z-z_{\tau}\right| \leq$ $R / 2 L_{R}$ and $\tau_{0}-\varepsilon \leq \tau \leq \tau_{0}$.

If we choose $\varepsilon$ so small that $\sqrt{M \varepsilon}<R / 4 L_{R}$, then we get $U \leq 2 R$ for all $(z, \tau)$ with $\left|z-z_{0}\right| \leq R / 4 L_{R}$ and $\tau_{0}-\varepsilon \leq \tau \leq \tau_{0}$.

Q. E. D.

Finally we can show that the $\gamma(\tau)$ converge to the straight line $r=\sqrt{n-1}$.

We shall prove that for any sequence $\tau_{j} \uparrow \infty$ there is a subsequence of $\tau_{j_{k}}$ for which $\gamma\left(\tau_{j_{k}}\right)$ converges to the line $r=\sqrt{n-1}$. Thus the only possible limit of the $\gamma(\tau)$ 's is the straight line, and it follows that the whole family $\gamma(\tau)$ converges to this line.

Consider therefore a sequence $\tau_{j} \uparrow \infty$. The lemmas 5.11-14 imply that there is a subsequence, which we shall again denote by $\gamma(\tau)$, such that the sequence of families of curves $\left\{\gamma\left(\tau_{j}+\tau\right):-1<\tau<1\right\}$ will converge in $C^{\infty}$, in bounded domains to a limiting family $\left\{\gamma^{*}(\tau):-1<\tau<1\right\}$, which also evolves with normal velocity $v=k+K(x, r, \theta)$. In fact, since the $\gamma(\tau)$ 's are graphs, the limit $\gamma^{*}(\tau)$ must be a graph $r=U^{*}(z, \tau)$, with $U^{*}$ a solution of (5.5) on some open subset $\mathcal{O} \subset \mathrm{R} \times(-1,1)$.

It follows from (5.7) that

$$
\int_{\tau_{R}+1}^{\infty} \int_{\gamma(\tau)} e^{-\left(x^{2}+r^{2}\right) / 2} r^{n-1}(k+K(x, r, \theta))^{2} d s d \tau<\infty
$$

and hence that

$$
\lim _{j \rightarrow \infty} \int_{\tau_{j}-1}^{\tau_{j}+1} \int_{\gamma(r)} e^{-\left(x^{2}+r^{2}\right) / 2} r^{n-1}(k+K(x, r, \theta))^{2} d s d \tau=0
$$

so that $k+K(x, r, \theta)=0$ on $\gamma^{*}(\tau)$. Thus the $\gamma^{*}(\tau)$ are independent of $\tau$, and are geodesics of the metric $\chi_{n}$ in the upper halfplane. 


\section{Mean Curvature Flow Through Singularities for Surfaces of Rotation.}

Equivalently, the function $U^{*}(z, \tau)$ is independent of $\tau$ (so we may write it as $U^{*}(z)$ ) and it satisfies (5.8) on its domain of definition.

The problem now is to prove that $U^{*}$ must in fact be constant. We know that it satisfies

$$
U^{*}(z) \geq \sqrt{\delta(n-1)}
$$

and, by combining $H \geq 0$ with equation (5.8) we find

$$
z U_{z}^{*}(z)-U^{*}(z) \leq 0
$$

Furthermore, if $(a, b) \subset \mathbf{R}$ is a component of the domain of $U^{*}$, then it follows from lemmas 5.11-14 that

(*) either $a=-\infty$, or else $U^{*}(z) \rightarrow \infty$ as $z \downarrow a$; likewise either $b=\infty$, or $U^{*}(z) \uparrow \infty$ as $z \uparrow b$.

5.15. Proposition. The only solution of (5.8-10) which satisfies the condition $(*)$ is the constant $U^{*}(z) \equiv \sqrt{n-1}$.

This proposition completes the proof of the blow-up theorem. We have a proof of this theorem, but we shall not present it here, since it is rather tedious, and Huisken [H3] has proved a much more general result: his result implies that any embedded hypersurface with $H \geq 0$ obtained in a blow-up procedure either is a sphere $S^{n}$, a product of a sphere and a flat subspace $S^{k} \times \mathrm{R}^{n-k}$. In particular, Huisken's result does not assume any kind of symmetry.

Our proof of the proposition runs along the following lines. In [H2] Huisken showed that the only compact selfsimilar solution to the mean curvature flow with positive mean curvature is the sphere. His proof consists of two parts: In the first he derives an equation similar to (5.2) for the quantity $Z=|\mathrm{A} / H|^{2}$, and using the compactness of the hypersurface he concludes from the strong maximum principle that $Z$ must be constant. Then, a purely local analysis of the fact that $Z$ is constant shows that the hypersurface either must be a sphere $S^{n}$, or the product of $S^{k}$ and $\mathrm{R}^{n-k}$, or else the product of an Abresch-Langer curve with $R^{n-1}$.

This second and third alternatives are ruled out in [H2] since Huisken assumes his hypersurface to be compact. Our blown-up hypersurface is noncompact, and if we could modify Huisken's proof so it would apply to our situation, then we could rule out the first alternative (because it leads to a compact blow-up), as well as the third (which is not embedded). Hence we would find that the blow-up is a cylinder $S^{1} \times \mathrm{R}^{n-1}$, i.e. that the function $U^{*}$ is constant.

The only time where Huisken uses the compactness of the blow-up in [H2] is when he applies the maximum principle to the equation for $Z$. The tedious part of the proof which we have omitted consists of an asymptotic analysis of $Z$ as $z \rightarrow a$ or $z \rightarrow b$ : we find that $Z \rightarrow(n-1)^{-1 / 2}$; we also find points $z \in(a, b)$ at which $Z \geq(n-1)^{-1 / 2}$. Thus, $Z$ must either be constant, or attain an interior maximum. But the maximum principle precludes 


\section{Altschuler, Angenent and Giga}

the latter from occuring, so that $Z$ must be constant, and Huisken's arguments may be used to conclude that the blow-up must be a cylinder.

The inner evolution at time $t=T$. So far we have studied the behaviour of the smooth hypersurfaces $\Gamma(t)$ as $t \uparrow T$. We conclude this section by taking a look at the corresponding inner and outer evolutions.

Let $D$ be a rotationally symmetric inner evolution with $\partial D(0)=\Gamma(0)$ smooth and compact. We shall assume that $D$ is connected, but if $D$ has more than one component then the following may be applied to each of those components.

Given $D$, let $E$ be the outer evolution with $E(0)=\bar{D}(0)$, and put $\Gamma=E \backslash D$. Denote the smallest $t>0$ at which $\partial D(t)$ fails to be smooth by $T>0$. As we pointed out in the beginning of this section, a $T_{1}<T$ exists such that the number of necks of $\Gamma(t)$ is constant, say $m$, for $T_{1}<t<T$. For $T_{1}<t<T$ we can define $a(t), b(t), \xi_{j}(t), \eta_{j}(t)$ as before. For $0 \leq t<T$ the domain $D(t)$ is given by $\{(x, y):|y|<u(x, t)\}$. By the limit surface lemma the function $u$ extends to a continuous function on $\mathrm{R} \times[0, T]$. Define

$$
\begin{aligned}
& D_{*}=\{(x, y):|y|<u(x, T), x \in \mathrm{R}\} \\
& E_{*}=\{(x, y):|y| \leq u(x, T), a(T) \leq x \leq b(T)\}
\end{aligned}
$$

5.16. Lemma. $D(T)=D_{*}$ and $E(T)=E_{*}$.

Proof. The continuity lemma 3.6 implies that $E(t)$ converges to $E(T)$ in the Hausdorff metric, as $t \uparrow T$. But the continuity of $u(x, t)$ at $t=T$ implies that $E(t)$ also converges to $E_{*}$. Hence $E(T)=E_{*}$.

The dual argument leads to $D(T)=D_{*}$.

Q.E. D.

It may happen that $D(T)$ is empty: in this case $a(T)=b(T)$, and $E(T)$ consists of one point.

If $D(T)$ is not empty then $a(T)<b(T)$, and $\Gamma(t)$ must have had at least one neck during $\left(T_{1}, T\right)$, i.e. $m \geq 1$ (we may conclude this from lemma 5.6.)

Choose a maximal sequence $1 \leq j_{1}<j_{2}<\cdots<j_{k} \leq m$ with $u\left(\xi_{j_{l}}(T), T\right)=0$ and $a(T)<\xi_{j_{1}}(T)<\cdots<\xi_{j_{k}}(T)<b(T)$. Put

$$
\begin{gathered}
x_{l}=\xi_{j_{l}}(T) \text { for } 1 \leq l \leq k \\
x_{0}=a(T), x_{k+1}=b(T) \\
U_{l}=\left\{(x, y):|y|<u(x, T), x_{l-1}<x<x_{l}\right\}, \quad l=1,2, \ldots, k+1 .
\end{gathered}
$$

The number of singularities $h$ of $D(T)$ is the number of points $\left\{\xi_{j}(T)\right\}$ such that $u\left(\xi_{j}(T), T\right)=0$. Note that $k \leq h \leq k+2$. 
Mean Curvature Flow Through Singularities for Surfaces of Rotation.

5.17. Lemma. $D(T)=U_{1} \cup \cdots \cup U_{k+1}$, and each $U_{l}$ is an $\alpha$-domain for some $\alpha>0$. If $\partial U_{l}$ has $m_{l}$ necks, then $\sum m_{l} \leq m-h$ and $h \leq k$.

Proof. It is clear that $D(T)$ may be written as the union of the $U_{i}$ 's. Choose $\alpha>0$ so small that $u\left(\xi_{j}(T), T\right)>\alpha$ for any $j=1,2, \cdots, m$ with $u\left(\xi_{j}(T), T\right)>0$ (necks which have not yet pinched). Then $u(\cdot, T)$ is smooth on $\left(x_{l-1}, x_{l}\right)$ and $u_{x}(x, T) \neq 0$ whenever $0<u(x, T)<\alpha$ : hence $U_{l}$ is an $\alpha$-domain.

Q. E. D.

Thus we have shown that at the time of singularity the inner evolution $D(T)$ is the union of a finite number $k+1$ of $\alpha$-domains, for some $\alpha>0$; this number $k+1$ is bounded by $m+1$, where $m$ is the number of necks of $\partial D(t)$, for $t$ sufficiently close to $T(t<T)$.

\section{§6. After the singularity.}

Instant smoothness. The main result of this section states that $\alpha$-domains which are smooth away from the $x$-axis, but may have singularities on the $x$-axis, become smooth immediately as they evolve by their mean curvature.

6.1. Theorem. If $U$ is an $\alpha$-domain, and $D \subset[0, \infty) \times \mathrm{R}^{n+1}$ is the corresponding inner evolution by mean curvature, then there is a $T>0$ such that $\partial D(t)$ is a smooth hypersurface for $0<t \leq T$.

In fact, the second fundamental form and all its covariant derivatives are uniformly bounded on any time interval $[\delta, T]$ with $\delta>0$.

Away from the axis the hypersurfaces $\partial D(t)$ are uniformly smooth up to $t=0$, in the sense that for any $\rho>0$, the second fundamental form and its derivatives are uniformly bounded as long as $r \geq \rho, 0<t \leq T$.

One may interpret this theorem as a local existence theorem for a classical solution where the prescribed initial datum is singular. Since the normal velocity $v$ of the surface $\partial D(t)$ coincides with its mean curvature, the theorem implies that its covariant derivatives $\nabla^{j}(v)$ are uniformly bounded for any $j \geq 0$ on any time interval $[\delta, T]$ with $\delta>0$. One concludes from this that the $n+1$ dimensional hypersurface in $(0, T) \times \mathrm{R}^{n+1}$ given by $D^{T}=D \cap(0, T) \times \mathrm{R}^{n+1}$ actually is smooth, and that it may also be written as

$$
D^{T}=\bigcup_{0<t<T}\{t\} \times \partial D(t)
$$

To prove the instant smoothness theorem, we first approximate $U$ by a nondecreasing sequence $\left\{U_{j}\right\}_{j=1}^{\infty}$ of smoothly bounded $\alpha$-domains, obtained by smoothing $U$ near the axis. If the $D_{j}$ are the inner solutions with initial data $U_{j}$, then it was shown in section 3 that the sequence $D_{j}$ approximates $D$ from the inside, i.e. $D_{j} \nearrow D$. 


\section{Altschuler, Angenent and Giga}

We then show that for some $t_{0}>0$, and $0<\beta<\alpha$ the $D_{j}(t)$ are $\beta$-domains for $t \in\left(0, t_{0}\right)$. This is a crucial step in the proof, and we rely on the Sturmian theorem to establish it.

Using the Evans-Spruck estimates, one can prove that the $\partial D_{j}(t)$ are uniformly smooth for $0<t<t_{0}$ near the axis of rotation, and conclude the smoothness of their limit $\partial D(t)$. We wrap up the proof by showing that $\partial D(t)$ is also smooth away from the axis, which actually turns out to be easier to prove than the smoothness near the axis.

6.2. Lemma. Let $U$ be an $\alpha$-domain in $\mathrm{R}^{n+1}$. There is a sequence of smoothly bounded $\alpha$-domains $\left\{U_{j}\right\}_{j=1}^{\infty}$ represented by $u_{j} \in C(\mathbf{R})$ such that $U_{j} \nearrow U$.

Proof of LEMMA 6.2. Let $a_{1}, a_{2}$ be the end points of $U$ and choose $\delta_{1}, \delta_{2}$ as in the definition of $\alpha$-domains given in $\$ 2$. We set

$$
a_{1, j}=a_{1}+\frac{\delta_{1}}{2^{j}}, \quad a_{2, j}=a_{2}-\frac{\delta_{2}}{2^{j}}
$$

and

$$
u_{j}(x)= \begin{cases}u_{0}(x) & \text { if } a_{1, j} \leq x \leq a_{2, j} \\ 0 & \text { if } x \leq a_{1, j+1} \text { or } x \geq a_{2, j+1}\end{cases}
$$

On the intervals $\left(a_{1, j+1}, a_{1, j}\right)$ and $\left(a_{2, j}, a_{2, j+1}\right)$ we choose $u_{j}$ so that

$$
\begin{aligned}
& u_{j} \leq u_{0}, \quad u_{j} \in C^{\infty}\left(\left(a_{1, j+1}, a_{2, j+1}\right)\right), \quad u_{j} \in C(\mathrm{R}) \\
& u_{j}^{\prime}>0 \text { on }\left(a_{1, j+1}, a_{1, j}\right) \\
& u_{j}^{\prime}<0 \text { on }\left(a_{2, j}, a_{2 j+1}\right)
\end{aligned}
$$

and that $U_{j}=\left\{(x, y) \in \mathrm{R}^{n+1} ; \quad r<u_{j}(x)\right\}$ has smooth boundary. (This is, of course, possible.) By the definition of $u_{j}$ we see $u_{j} \leq u_{j+1}$ and $\lim _{j \rightarrow \infty} u_{j}=u_{0}$ which implies $U_{j} \nearrow U$. One easily sees that the $U_{j}$ are $\alpha$-domains.

Q. E. D.

Denote the cylinder $\{(x, y):|y|<\rho\}$ by $C_{\rho}$. At time $t>0$, the inner evolution of such a cylinder is given by $C_{r(t, \rho)}$, for $t<t_{*}(\rho)$; it is empty for $t \geq t_{*}(\rho)$. Here

$$
\begin{gathered}
t_{*}(\rho)=\frac{\rho^{2}}{2(n-1)} \\
r(t, \rho)=\sqrt{\rho^{2}-2(n-1) t} .
\end{gathered}
$$

For any $\alpha$-domain $V$ with $\partial V$ smooth let $D$ be the inner evolution with $D(0)=V$, and define $T_{V}$ to be the smallest $t>0$ at which $\partial D(t)$ is no longer smooth. By the local existence theorem for smooth solutions of the mean curvaure flow, $T_{V}>0$. 


\section{Mean Curvature Flow Through Singularities for Surfaces of Rotation.}

6.3. Lemma. There exists a $t_{V}^{\alpha} \in\left(0, T_{V}\right]$ such that $D(t)$ is an $\alpha(t)$-domain for all $0<t<t_{V}^{\alpha}$, where $\alpha(t)=\sqrt{\alpha^{2}-2(n-1) t}$.

If $W \supset V$ is another smoothly bounded $\alpha$-domain then we can choose $t_{W}^{\alpha}>t_{V}^{\alpha}$. In particular, it follows that $T_{W} \geq t_{V}^{\alpha}$.

Proof. Since $V$ is not contained in the cylinder $\bar{C}_{\alpha}$, there is a small ball $B_{\varepsilon}(P)$ contained in $V$ but disjoint from $\bar{C}_{\alpha}$. The inclusion property for inner evolutions implies that $D(t)$ contains the ball $B_{\epsilon(t)}(P)$ for $0<t<\varepsilon^{2} / 2 n$, where $\varepsilon(t)=\sqrt{\varepsilon^{2}-2 n t}$.

We define

$$
t_{V}^{\alpha}=\min \left\{\frac{\varepsilon^{2}}{2 n}, \frac{\alpha^{2}}{2(n-1)}\right\} .
$$

If $W$ is a larger $\alpha$-domain then the same ball $B_{e}(P)$ will be contained in $W \backslash \bar{C}_{\alpha}$ so that the following arguments apply to $W$ in the same way as they are stated for $V$, provided we choose $t_{W}^{\alpha}=t_{V}^{\alpha}$. Thus it will suffice to prove that the first part of the lemma holds with this choice of $t_{V}^{\alpha}$.

We shall first show that $D(t)$ is an $\alpha(t)$-domain for $0 \leq t<\min \left\{T_{V}, t_{V}^{\alpha}\right\}$. As long as $t<T_{V}$ holds the first two conditions in the definition of an $\alpha$-domain are clearly fulfilled, so all we have to show is that $\partial D\left(t_{0}\right)$ intersects each $C_{\rho}$ with $0<\rho<\alpha\left(t_{0}\right)$ exactly twice if $t_{0}<\min \left\{T_{V}, t_{V}^{\alpha}\right\}$.

Let $0<\rho<\alpha\left(t_{0}\right)$ and $0<t_{0}<\min \left\{T_{V}, t_{V}^{\alpha}\right\}$ be given, and define

$$
\rho(t)=\sqrt{\rho^{2}-2(n-1)\left(t-t_{0}\right)}=r\left(t-t_{0}, \rho\right) .
$$

Since $D(t)$ contains the ball $B_{\varepsilon(t)}(P)$, and since this ball lies outside of the cylinder $C_{\alpha}$, $\partial D(t)$ must intersect each $\partial C_{\rho}$ with $0<\rho<\alpha$ at least twice, for any $0<t<t_{V}^{\alpha}$.

On the other hand $\partial D(t)$ and $\partial C_{p(t)}$ are smooth families of hypersurfaces evolving by their mean curvature, so the number of their intersections does not increase with time; moreover, whenever they have a nontransverse intersection this number will decrease. At $t=0$ we have $\rho(t)<\alpha$ so $\partial V=\partial D(0)$ and $\partial C_{\rho(0)}$ intersect exactly twice, because $V$ is an $\alpha$-domain. At $t=t_{0} \partial D(t)$ and $\partial C_{\rho(t)}$ therefore intersect at most twice.

We therefore find that $\partial D\left(t_{0}\right)$ and $\partial C_{\rho\left(t_{0}\right)}$ intersect exactly twice. By the same arguments the number of intersections is two for all $t$ near $t_{0}$, so that it does not decrease at $t=t_{0}$; hence these intersections must be transverse.

So far we have established that $D(t)$ is an $\alpha(t)$-domain for $t<\min \left\{t_{V}^{\alpha}, T_{V}\right\}$. It remains to be shown that $T_{V} \geq t_{V}^{\alpha}$.

Assume that $T_{V}<t_{V}^{\alpha}$. Then $\partial D(t)$ does not shrink to a point, for $D(t)$ contains the ball $B_{e(t)}(P)$. By lemma 5.5 and 5.6 one of the necks of $\partial D(t)$ must therefore collapse. This cannot happen either, since $D(t)$ is an $\alpha(t)$-domain for $0<t<T_{V}$, and $\alpha(t) \geq \alpha\left(T_{V}\right)>0$, by virtue of our assumption $T_{V}<t_{V}^{\alpha}$. This assumption must therefore be incorrect.

Q.E. D.

Consider the $U_{j}$ 's again, and let $D_{j}$ be the inner evolutions with $D_{j}(0)=U_{j}$. By the foregoing lemma there exist $t_{0}, \delta>0$ such that all $D_{j}(t)$ are $\delta$-domains with smooth boundaries, for $j \geq 1,0 \leq t \leq t_{0}$. 


\section{Altschuler, Angenent and Giga}

6.4. Lemma. There is a $t_{1} \in\left(0, t_{0}\right)$ such that for any $t_{2} \in\left(0, t_{1}\right)$ the second fundamental form of $\partial D_{j}(t)$ and all its derivatives are uniformly bounded for $j \geq 1, t_{2}<t<t_{1}$.

Proof. Let $D(t)$ and $D_{j}(t)$ be obtained by rotating the graphs of $r=u(x, t)$, and $r=u_{j}(x, t)$ about the $x$-axis. Choose $p, q \in \mathbf{R}, p<q$ so that $u(p, 0)=u(q, 0)=\alpha / 2$, and consider the points

$$
\begin{aligned}
P & =(p, 0, \ldots, 0), & Q=(q, 0, \ldots, 0), \\
P^{\prime} & =(p, \alpha, \ldots, 0), & Q^{\prime}=(q, \alpha, \ldots, 0) .
\end{aligned}
$$

Then $P, Q \in D(0)$ and $P^{\prime}, Q^{\prime} \in \bar{D}(0)^{\mathrm{c}}$, so for some $\varepsilon \in(0, \delta)$ we even have $\overline{B_{\varepsilon}(P)} \cup \overline{B_{\varepsilon}(Q)} \subset$ $D(0)$ and $B_{\varepsilon}\left(P^{\prime}\right) \cup B_{\varepsilon}\left(Q^{\prime}\right) \subset D(0)^{\mathrm{c}}$. By the comparison and separation theorems we get

$$
\begin{gathered}
\overline{B_{\varepsilon(t)}(P)} \cup \overline{B_{\varepsilon(t)}(Q)} \subset D(t) \\
B_{\varepsilon(t)}\left(P^{\prime}\right) \cup B_{\varepsilon(t)}\left(Q^{\prime}\right) \subset D(t)^{c}
\end{gathered}
$$

for $0 \leq t \leq \min \left(t_{0}, \varepsilon^{2} / 2 n\right)$, where $\varepsilon(t)^{2}=\varepsilon^{2}-2 n t$.

Since $D_{j} \uparrow D$ we may assume, after discarding a finite number of $D_{j}$ 's if necessary, that (6.2) and (6.3) also hold with $D(t)$ replaced by $D_{j}(t)$, for any $j$.

Let $t_{1}=\min \left(t_{0}, \varepsilon^{2} / 4 n\right)$. Then $\varepsilon(t) \geq \varepsilon / \sqrt{2}$ for $0 \leq t \leq t_{1}$, and it follows from (6.2) applied to $D_{j}(t)$ that $u_{j}(x, t) \geq \varepsilon / \sqrt{2}$ for $0 \leq t \leq t_{1}$ and $|x-p|<\varepsilon / \sqrt{2}$ or $|x-q|<\varepsilon / \sqrt{2}$. Since the $D_{j}(t)$ 's are all $\delta$-domains, and $\delta>\varepsilon$, we find that

$$
u_{j}(x, t) \geq \varepsilon / \sqrt{2} \text { on } \Omega=(p-\varepsilon / \sqrt{2}, q+\varepsilon / \sqrt{2}) \times\left(0, t_{1}\right) .
$$

The $u_{j}$ satisfy the horizontal graph equation on $\Omega$, so by the interior estimates from theorem 4.3(b) $u_{j, x}$ and all higher derivatives of the $u_{j}$ are uniformly bounded on any subdomain $\Omega^{\prime}=[p, q] \times\left[t_{2}, t_{1}\right] \subset \Omega$. This implies that for any $0<t_{2}<t_{1}$ the second fundamental forms of the $\partial D_{j}(t)$ and their derivatives are uniformly bounded for $t_{2} \leq t \leq t_{1}$ and $p \leq x \leq q$.

For a complete proof we must also provide such an estimate in the regions where $x \leq p$ or $x \geq q$. We shall only consider the region $x \leq p$, the other situation being similar.

It follows from (6.3), that all $D_{j}(t)$ 's are $\delta$-domains for $0<t \leq t_{0}$, and our choice of $t_{1}$ that

$$
u_{j}(x, t) \leq \delta-\frac{\varepsilon}{\sqrt{2}} \text { for } x \leq p, 0 \leq t \leq t_{1} .
$$

Thus for $0 \leq t \leq t_{1}$ the part of $\partial D_{j}(t)$ on which $x \leq p$ is contained in the left $\delta$-cap of $\partial D_{j}(t)$, i.e. one of the two components of $\partial D_{j}(t) \cap\{(x, y):|y|<\delta\}$. We may represent this left cap as a full graph $x=w_{j}(y, t)$, with $w_{j}: B_{\delta}(0) \times\left[0, t_{1}\right] \rightarrow \mathrm{R}$ a smooth solution of the full graph equation. The Evans-Spruck estimates then imply that all derivatives $\nabla^{k} w_{j}(y, t)$ are uniformly bounded on $B_{\delta-\varepsilon / \sqrt{2}}(0) \times\left[t_{2}, t_{1}\right]$ for any $t_{2}>0$. This implies that the second fundamental form of $\partial D_{j}(t)$ and its derivatives are uniformly bounded when $x \leq p, t_{2} \leq t \leq t_{1}$.

Q.E. D. 


\section{Mean Curvature Flow Through Singularities for Surfaces of Rotation.}

Proof of THEOREM 6.1. The smoothness of $D$ in $\left(0, t_{1}\right)$ follows from lemma 6.4. It remains to prove the smoothness of $D$ in $\left[0, t_{1}\right)$ away from the $x$-axis. Note that the interior gradient estimate (theorem 4.3.b) is valid up to the initial value provided that the initial derivatives are bounded. Using the higher derivative estimates (lemma 2.4 (ii)), we observe that the second fundamental form of $\partial D_{j}(t)$ and all its derivatives are uniformly bounded away from the axis for $0 \leq t<t_{1}$ in lemma 6.4. This proves the smoothness of $D$ away from the axis.

Q. E. D.

\subsection{Corollary.}

(i.) The set $D(t)$ in theorem 6.1 is a $\delta$-domain with some $\delta>0$ for $0 \leq t \leq T$.

(ii.) $\partial D(t)$ is a continuous function of $t \in[0, T]$ with respect to the Hausdorff metric.

ProOF.

(i.) By lemma 6.4 all $D_{j}(t)$ are $\delta$-domains for $j \geq 1,0 \leq t<t_{0}$ if $t_{0}$ and $\delta$ are chosen sufficiently small. By lemma 6.4 this property is inherited by $D(t)$. Since $D(t)$ is smooth for $0<t \leq T$, part (i) now follows by choosing $\delta$ sufficiently small.

(ii.) By lemma 6.4 we only need to prove that $\partial D(t)$ moves continuously as $t \downarrow 0$ near singularities of $D(0)$. We may assume that the caps of $D(t), D_{j}(t)$ (resp.) are expressed as $x=v(r, t), x=v_{j}(r, t)$ (resp.) for $r \leq \delta, 0 \leq t \leq t_{0}$ where $t_{0}>0, \delta>0$ are independent of $j$. We consider only right caps because the proof for left caps is similar.

All we have to prove is the continuity of $v$ at $(0,0)$. The monotonicity of $v_{j}$ in $r$ and the moving cap lemma yields

$$
v_{j}(r, t) \leq v_{j}(0, t) \leq v_{j}(0,0)
$$

for $t \geq 0, r \geq 0$. Letting $j \rightarrow \infty$ yields

$$
v(r, t) \leq v(0,0) .
$$

By the continuity of $v_{j}$ at $(0,0)$ and $v_{j} \leq v$, we see

$$
\begin{aligned}
v_{j}(0,0) & =\lim _{t \downarrow 0 r 10} v_{j}(r, t) \leq \lim _{t \downarrow 0} \inf v(r, t) \\
& \leq \lim _{t \downarrow 0 r 10} \sup v(r, t) \leq v(0,0) .
\end{aligned}
$$

Letting $j \rightarrow 0$ yields

$$
v(0,0)=\lim _{t \downarrow 0 r \downarrow 0} v(r, t) .
$$

Q. E. D. 


\section{Altschuler, Angenent and Giga}

The complete inner evolution. Let $D$ be a rotationally symmetric inner evolution, with $D(0)$ smooth and connected. Denote the first time at which $\partial D(t)$ is non smooth by $t_{1}>0$. Choose some small $t \in\left(0, t_{1}\right)$ : at this time $\partial D(t)$ will have a finite number of necks, say $m \geq 0$.

If $D\left(t_{1}\right)$ is not empty then, by lemma 5.17 we may write $D\left(t_{1}\right)$ as a disjoint union of $k+1 \alpha$-domains $U_{1}, \ldots, U_{k+1}$, each of which has at most $m-k$ necks. By the separation theorem 3.5 the inner evolution $D$ after $t=t_{1}$ is the disjoint union of the inner evolutions corresponding to the $U_{l}$. We have just shown that these are smooth on some short time interval $\left(t_{1}, t_{1}+\varepsilon_{1}\right)$. Therefore we may apply the same arguments to these inner evolutions, and by induction on the number of necks we find that $D$ is singular at at most $m$ times $0<t_{1}<t_{2}<\cdots<t_{m}$, and that $\partial D$ is indeed smooth away from the $x$-axis.

\section{§7. Inner and Outer Evolutions coincide.}

In this section we shall show that $\bar{D}=E$ for any pair of inner and outer evolutions $D$ and $E$ with $\bar{D}(0)=E(0)$, and with $\partial D(0)$ smooth compact and rotationally symmetric. We have already shown that $\partial D(t)$ is smooth, except at finitely many times $0<t_{1}<t_{2}<\cdots<t_{m}$. Suppose that $\bar{D}=E$ in $[0, T]$ for some $t_{j}<T<t_{j+1}$. Then $\partial D(T)=\partial E(T)$ is smooth and it follows that $\bar{D}=E$ in $\left[0, t_{j+1}\right]$. If in this situation we could show that for some small $\varepsilon>0$ we also have $\bar{D}=E$ in $\left[0, t_{j+1}+\varepsilon\right]$, then we would be in the previous situation, and an induction argument would lead to $\bar{D}=E$ for all time.

Thus the problem is to show that if $D(0)$ is the union of a finite number of $\alpha$-domains, one may conclude that $\bar{D}=E$ for $0 \leq t \leq \varepsilon$, for some $\varepsilon>0$. In what follows we shall first show that such a conclusion is indeed warranted if $D(0)$ consists of one $\alpha$-domain, and then we use the technical regularity lemma 3.11 to deal with the general case.

Short time regularity for $\alpha$-domains. Let $U=\{(x, y):|y|<u(x)\}$ be an $\alpha$-domain, with inner and outer evolutions $D$ and $E$, respectively.

\subsection{Lemma. For some $\varepsilon>0$ we have $\bar{D}=E$ in $[0, \varepsilon]$.}

We begin the proof by observing that $\partial U$ is homeomorphic to a sphere. If $\partial U$ were smooth, then we could use the tubular neighborhood theorem to represent any hypersurface $C^{1}$ close to $\partial U$ by a graph over $\partial U$. Since $\partial U$ is not smooth we must resort to a slightly different construction, in order to achieve a similar representation of hypersurfaces "close" to $\partial U$.

Choose a smooth vector field $X: \mathrm{R}^{n+1} \rightarrow \mathrm{R}^{n+1}$ such that

(i) At any point $P \in \partial U$ not on the $x$-axis one has $\langle X(P), \mathbf{n}(P)\rangle<0$, where $\mathbf{n}(P)$ is the inward unit normal at $P$;

(ii) Near the two endpoints of $\partial U, X$ is constant, with $X \equiv \pm \mathrm{e}_{0}=( \pm 1,0, \ldots, 0)$.

One can construct such a vectorfield from the unit normal $\mathbf{n}$ and the constant vector 
Mean Curvature Flow Through Singularities for Surfaces of Rotation.

fields $\pm e_{0}$ by using a partition of unity.

Since $X \neq 0$ on the compact set $\partial U$, there is an open neighborhood $V \supset \partial U$ on which $|X| \geq \delta>0$ for some $\delta>0$.

7.2. Proposition. For small enough $\rho>0$ there exists a hypersurface $\Sigma \subset V$ with

(i) $X(P) \notin T_{P} \Sigma$ at all $P \in \Sigma$, i.e. $\Sigma$ is transverse to the vectorfield $X$;

(ii) $\Sigma=\partial U$ in $\{(x, y):|y| \geq 2 \rho\}$;

(iii) $\Sigma \cap\{(x, y):|y| \leq \rho\}$ consists of two flat disks $\Delta_{a}=\{(a, y):|y| \leq \rho\}$ and $\Delta_{b}=$ $\{(b, y):|y| \leq \rho\}$ for some $a<b$.

Proof. See figure 7.1

Q. E. D.

Figure 7.1-The vectorfield and the hypersurface.

The construction of $\Sigma$ is similar to that of the inner approximations $U_{j} \subset U$ of lemma 6.2. The only difference is that we have added the hypothesis that $\Sigma$ is flat near the $x$-axis. If $\phi^{t}: \mathrm{R}^{n+1} \rightarrow \mathrm{R}^{n+1}(t \in \mathrm{R})$ denotes the flow generated by the vectorfield $X$ on $\mathrm{R}^{n+1}$, then we define $\sigma: \Sigma \times \mathrm{R} \rightarrow \mathrm{R}^{n+1}$ by

$$
\sigma(P, s)=\phi^{s}(P) \text {. }
$$

It follows from proposition 7.2(i) that $\sigma$ is a diffeomorphism onto its image.

For any $C^{1}$ function $u: \Sigma \rightarrow \mathrm{R}$ the image of the graph of $u$ under $\sigma$ is a $C^{1}$ hypersurface in $\mathrm{R}^{n+1}$, parametrized by $P \mapsto \sigma(P, u(P))$. Conversely, any hypersurface which is $C^{1}$ close to $\Sigma$ can be obtained in this way. Although $\partial U$ is not smooth, our construction is such that it can still be represented by the graph of a continuous function $u: \Sigma \rightarrow R$, i.e. $\sigma^{-1}(\partial U)$ is the graph of $u$. Indeed, near the axis $u(P)$ is simply the horizontal distance from the point $P$ on $\Sigma$ to the nearest point $P^{\prime}$ on $\partial U$ which has the same distance to the $x$-axis (see figure 7.1).

Suppose $\Gamma(t)(0<t<T)$ are smooth hypersurfaces with $\sigma^{-1}(\Gamma(t))$ the graph of $u(\cdot, t)$, for some smooth function $u: \Sigma \times(0, T) \rightarrow \mathrm{R}$. Let $z_{1}, \ldots, z_{n}$ be local coordinates on an open subset of $\partial U$. Then in these coordinates mean curvature flow is equivalent to the following quasilinear PDE

$$
\frac{\partial u}{\partial t}=a^{i j}(z, u, \nabla u) \frac{\partial^{2} u}{\partial z_{i} \partial z_{j}}+b(z, u, \nabla u) .
$$

Here $a^{i j}$ and $b$ are smooth functions of their arguments (cf. [A3, section 3]). These functions depend on the particular choice of coordinates $z_{1}, \ldots, z_{n}$. Nevertheless, the $a^{i j}$ are always positive definite so that (7.1) is a parabolic equation. 


\section{Altschuler, Angenent and Giga}

Near the $x$-axis $\Sigma$ is flat, so on the two disks $\Delta_{a, b}$ the Euclidean coordinates $y_{1}, \ldots, y_{n}$ form a local coordinate system on $\Sigma$. Since $X= \pm e_{0}$ when $r<\rho$, the map $\sigma$ is simply given by

$$
\sigma\left(y_{1}, \ldots, y_{n}, s\right)= \begin{cases}\left(a+s, y_{1}, \ldots, y_{n}\right) & \text { on } \Delta_{a} \\ \left(b-s, y_{1}, \ldots, y_{n}\right) & \text { on } \Delta_{b}\end{cases}
$$

In these coordinates equation (7.1) reduces to the full graph equation $u_{t}=g^{i j}(\nabla u) \nabla_{i} \nabla_{j} u$.

7.3. Proposition. Let $\Gamma_{1}(t), \Gamma_{2}(t)(0 \leq t<T)$ be two families of hypersurfaces with $\sigma^{-1}\left(\Gamma_{j}(t)\right)$ the graph of $u_{j}(\cdot, t)$ for certain $u_{j} \in C^{0}(\Sigma \times[0, T))$. Assume that the $u_{j}$ are smooth on $\Sigma \times(0, T)$ as well as on $\left(\Sigma \backslash \Delta_{a} \cup \Delta_{b}\right) \times[0, T)$. Then if the $\Gamma_{j}(t)$ evolve by their mean curvature and if $\Gamma_{1}(0)=\Gamma_{2}(0)$, one has $\Gamma_{1}(t)=\Gamma_{2}(t)$ for $0<t<T$.

Proof. Consider $v(P, t)=u_{1}(P, t)-u_{2}(P, t)$. Our assumptions imply that $v \in$ $C^{0}(\Sigma \times[0, T))$, and that $v$ is smooth on $\left(\Sigma \backslash \Delta_{a} \cup \Delta_{b}\right) \times[0, T)$, as well as $\Sigma \times(0, T)$. Moreover $v(P, 0) \equiv 0$.

Define $m(t)=\max \{v(P, t): P \in \Sigma\},(0 \leq t<T)$, and for each $0 \leq t<T$ with $m(t)>0$ choose a $P_{t} \in \Sigma$ with $u\left(P_{t}, t\right)=m(t)$. We shall now show that $\bar{m}^{\prime}(t) \leq C m(t)$ for some constant $C<\infty$.

Case 1. Suppose that $P_{t} \in \Delta_{a} \cup \Delta_{b}$, in fact, for definiteness assume $P_{t} \in \Delta_{a}$. Then in the $y_{1}, \ldots, y_{n}$ coordinates the $u_{j}$ satisfy the full graph equation, which is of the form $u_{t}=F\left(\nabla u, \nabla^{2} u\right)$. Hence $v=u_{1}-u_{2}$ satisfies a linear parabolic equation $v_{t}=a_{i j}(y, t) \nabla_{i} \nabla_{j} v+b_{i}(y, t) \nabla_{i} v$ where $\nabla_{i}=\frac{\partial}{\partial y_{i}}$. At $P_{t} v(\cdot, t)$ attains its maximum, so $\nabla_{i} v\left(P_{t}, t\right)=0$ and $\nabla_{i} \nabla_{j} v\left(P_{t}, t\right)$ is non positive. It follows that $v_{t} \leq 0$ and hence $m^{\prime}(t) \leq 0$.

Case 2. Assume that $P_{t} \in \Sigma \backslash \Delta_{a} \cup \Delta_{b}$. Then we can choose coordinates $z_{1}, \ldots, z_{n}$ on some neighborhood of $P_{t}$ and compute the corresponding functions $a^{i j}(z, u, \nabla u), b(z, u, \nabla u)$ for which $u_{1}$ and $u_{2}$ satisfy (7.1). We may write this equation as $u_{t}=F\left(z, u, \nabla u, \nabla^{2} u\right)$. Subtracting the equations for $u_{1}$ and $u_{2}$ we find that $v=u_{1}-u_{2}$ is a solution of a linear parabolic equation $v_{t}=a_{i j}(z, t) \nabla_{i} \nabla_{j} v+b_{i}(z, t) \nabla_{i} v+c(z, t) v$ where $c(z, t)$ is given by

$$
c(z, t)=\int_{0}^{1} F_{u}\left(z, u^{\theta}, \nabla u^{\theta}, \nabla^{2} u^{\theta}\right) d \theta, \quad \text { with } u^{\theta}=(1-\theta) u_{0}+\theta u_{1} .
$$

Outside of the disks $\Delta_{a, b}$ the function $u$ is smooth up to $t=0$, so the coefficient $c(z, t)$ is bounded, say by $|c(z, t)| \leq M<\infty$. The constant $M$ may depend on the coordinate system $z_{1}, \ldots, z_{n}$ we have chosen, but $\Sigma$ is compact, so we can cover $\Sigma \backslash \Delta_{a} \cup \Delta_{b}$ with a finite number of such coordinate neighborhoods: If we choose $M$ large enough, then the bound $|c| \leq M$ will hold in each of these coordinate neighborhoods.

We again have $\nabla_{i} v\left(P_{t}, t\right)=0$, and $\nabla_{i} \nabla_{j} v\left(P_{t}, t\right) \leq 0$, so we find that

$$
v_{t}\left(P_{t}, t\right) \leq c\left(P_{t}, t\right) v\left(P_{t}, t\right) \leq c\left(P_{t}, t\right) m(t) \leq M m(t) .
$$

Combining these two cases we come to the conclusion that $v_{t} \leq M m(t)$ at any point $P_{t}$ where $v\left(P_{t}, t\right)=m(t)>0$; and hence we also find that $m^{\prime}(t) \leq M m(t)$ for $0 \leq t<T$; and finally, since $m(0)=0$ we see that $m(t) \leq 0$, i.e. $v(P, t) \leq 0$ for all $P \in \Sigma, t \in[0, T)$. 


\section{Mean Curvature Flow Through Singularities for Surfaces of Rotation.}

Reversing the roles of $u_{1}$ and $u_{2}$ we also get $v \geq 0$, so that $v \equiv 0$, and $u_{1} \equiv u_{2}$.

Q. E. D.

Consider an $\alpha$-domain $U$, its inner evolution $D$ and the outer evolution $E$ of $\bar{U}$. We have shown in $\S 6$ that $\partial D(t)$ is smooth on some short time interval $0<t<T$. The same arguments may be used to show that $\partial E(t)$ is also smooth during a short enough time interval $(0, T)$. Indeed, instead of approximating $U$ from the inside one constructs a sequence of smoothly bounded $\alpha$-domains $U_{n}$ with $\bar{U}_{n} \downarrow \bar{U}$, and uses lemma 6.3 to show that their corresponding outer evolutions are uniformly smooth on a short time interval $(0, T)$.

Thus we have two families of smooth hypersurfaces, $\partial D(t)$ and $\partial E(t)$, which are smooth for $0<t<T$, which evolve by their mean curvatures, and which coincide for $t=0$. Moreover, both $\partial D(t)$ and $\partial E(t)$ bound $\delta$-domains for some small $\delta>0$, and for $0<t<T$. This implies that both hypersurfaces $\partial D(t)$ and $\partial E(t)$ are images of graphs under $\sigma$, at least on a shorter time interval $0<t<T^{\prime}$, with $0<T^{\prime} \leq T$. We may therefore apply our proposition and conclude that $\partial D(t)=\partial E(t)$, and hence $\bar{D}=E$ in $[0, T)$. So we have proved lemma 7.1.

We now direct our attention to domains which are finite disjoint unions of $\alpha$-domains.

Let $0 \leq u \in C^{0}(\mathrm{R})$ have compact support. Put $U=\{(x, y):|y|<u(x)\}$. Assume that there are $\xi_{0}<\xi_{1}<\cdots<\xi_{m+1}$ such that

$$
\begin{gathered}
\{x \in \mathbf{R}: u(x)>0\}=\left(\xi_{0}, \xi_{1}\right) \cup\left(\xi_{1}, \xi_{2}\right) \cup \cdots \cup\left(\xi_{m}, \xi_{m+1}\right), \\
u \mid\left(\xi_{j}, \xi_{j+1}\right) \text { is smooth for } j=0,1,2, \ldots, m, \\
\lim _{x \rightarrow \xi_{i}} \frac{u(x)}{\left|x-\xi_{j}\right|}=0 \quad j=1, \ldots, m .
\end{gathered}
$$

Denote the inner and outer evolutions of $U$ and $\bar{U}$ by $D$ and $E$, respectively; let $P_{j}$ be the point $\left(\xi_{j}, 0, \ldots, 0\right)$. It should be noted that any inner evolution $D$ with $D(0)$ an $\alpha$-domain satisfies (7.2-4) at any of its singular moments.

7.4. Lemma. $P_{j} \notin E(t)$ for any $t>0,1 \leq j \leq m$.

PROOF. In [A5] it was shown that there exists a closed curve $\gamma$ in the $x r$-upper halfplane such that $\Gamma(t)=\{(\lambda(t) x, \lambda(t) y):(x,|y|) \in \gamma\}$ with $\lambda(t)=\sqrt{-2 t}$ is a smooth solution of the mean curvature flow for $t<0$. In $\mathrm{R}^{3}$ it represents a torus-like surface which shrinks to the origin as $t \uparrow 0$.

Put $\varepsilon=\inf \{r /|x|:(x, r) \in \gamma\}$. Since $\gamma$ is compact and $r>0$ on $\gamma, \varepsilon$ is positive.

Choose $\delta>0$ so small that $u(x) \leq \frac{\varepsilon}{2}\left|x-\xi_{j}\right|$ for $\left|x-\xi_{j}\right|<\delta, j=1, \ldots, m$.

For any $\tau>0$ we define an outer evolution $F_{r, j}$ :

$$
F_{\tau, j}(t)= \begin{cases}\left\{\left(\xi_{j}+\lambda(t-\tau) x, \lambda(t-\tau) y\right):(x,|y|) \in \gamma\right\} & \text { if } 0 \leq t \leq \tau \\ \emptyset & \text { when } t>\tau\end{cases}
$$

If $\tau$ is small then $F_{\tau, j}(0)$ will be contained in the region where $\left|x-\xi_{j}\right|<\delta$ and $|y| \geq \varepsilon\left|x-\xi_{j}\right|$, so that $F_{\tau, j}(0)$ and $\bar{U}$ are disjoint. By the separation theorem $E(\tau)$ and $F_{\tau, j}(\tau)=\left\{P_{j}\right\}$ 


\section{Altschuler, Angenent and Giga}

are also disjoint. The lemma is therefore true if $t$ is small enough. But if $P_{j} \notin E\left(t_{0}\right)$ for some $t_{0}>0$, then compactness of $E$ implies that $E\left(t_{0}\right)$ is disjoint from the strip $\left\{(x, y):\left|x-\xi_{j}\right| \leq \rho\right\}$ for small enough $\rho>0$. By the separation theorem this will continue to hold for all $t>t_{0}$, so that $E(t)$ does not contain $P_{j}$ for any $t>t_{0}$ either.

Q.E. D.

7.5. Lemma. If $D, E$ and $U$ are as above, then for some $\varepsilon>0$ one has $\bar{D}=E$ for $0 \leq t<\varepsilon$.

Proof. Let $U_{j}$ be the $j^{\text {th }}$ component of $U$, i.e.

$$
U_{j}=\left\{(x, y) \in U: \xi_{j-1}<x<\xi_{j}\right\}, \quad 0<j \leq m+1 .
$$

We have just shown that the $U_{j}$ 's, being $\alpha$-domains, are regular on some short enough time interval $0 \leq t<\varepsilon$. We shall now construct a sequence $U_{\alpha}^{j}$ of open sets and a double sequence of times $t_{\alpha, l}>0$ to which the second regularity lemma 3.11 may be applied.

By lemma 7.4 $E(t)$ does not intersect any of the hyperplanes $x=\xi_{j}$ for $j=1, \ldots, m$. Since $E(t)$ is compact there even is a $\delta_{t}>0$ such that $E(t) \subset W_{t}$ where $W_{t}=W_{t}^{1} \cup \ldots \cup$ $W_{t}^{m+1}$, with

$$
W_{t}^{j}= \begin{cases}\left(\xi_{j-1}+\delta_{t}, \xi_{j}-\delta_{t}\right) \times \mathrm{R}^{n} & \text { for } j=2, \ldots, m \\ \left(\xi_{0}, \xi_{1}-\delta_{t}\right) \times \mathrm{R}^{n} & \text { for } j=1 \\ \left(\xi_{m}+\delta_{t}, \xi_{m+1}\right) \times \mathrm{R}^{n} & \text { for } j=m+1\end{cases}
$$

We may assume that $\delta_{t}$ is a continuous and increasing function of $t \geq 0$.

It follows from the continuity in time theorem that $E(t)$ is contained in a $\sqrt{2 n t}$ neighborhood of $E(0)=\bar{U}$, so we have

$$
E(t) \subset V_{t}^{1} \cup \cdots \cup V_{t}^{m+1} \text { where } V_{i}^{j}=W_{i}^{j} \cap \mathcal{N}_{\sqrt{2 n t}}(U) .
$$

Define

$$
U_{\alpha}^{j}=\bigcup_{l \geq \alpha} V_{2^{-1}}^{j}, \quad t_{l, \alpha}=\min \left(2^{-l}, 2^{-\alpha}\right) .
$$

Then the $U_{\alpha}^{j}$ are pairwise disjoint; they satisfy $U^{j} \subset U_{\alpha}^{j}$ while $\bar{U}_{\alpha}^{j} \downarrow \bar{U}^{j}$ as $\alpha \uparrow \infty$; and they also satisfy $E\left(t_{l, \alpha}\right) \subset U_{\alpha}^{1} \cup \cdots \cup U_{\alpha}^{m+1}$. Since these are precisely the conditions of lemma 3.11 , it follows that the inner evolution $D$ is regular on a short enough time interval.

Q. E. D.

We may now prove the results which are claimed in the introduction.

PROOF OF MAIN RESULTS IN INTRODUCTION. By corollary 5.10 and lemma 7.5 we see $\bar{D}$ is an outer evolution so that $\Gamma=\bar{D} \backslash D$. The picture of the complete inner evolution $D$ presented in $\S 6$ now yields theorem 1.1 and also the estimates of the number of singular times in corollary 1.3. Theorem 1.2 is obtained by a combination of the single point pinching lemma, the limit surface lemma, and lemma 5.16-5.17.

Q. E. D. 
Mean Curvature Flow Through Singularities for Surfaces of Rotation.

\section{$\S 8$. Hamilton's incredibly shrinking dumbbell.}

Consider the one parameter family of inner evolutions $D^{\lambda} \subset[0, \infty) \times \mathbf{R}^{n+1}$ whose initial domain $D^{\lambda}(0)$ is the inside of the smooth hypersurface $\Gamma^{\lambda} \subset \mathbf{R}^{n+1}$, where $\Gamma^{\lambda}$ is defined by the equation

$$
r^{2}=\left(1-x^{2}\right)\left(1-\lambda+\lambda x^{2}\right)^{2} .
$$

For $\lambda=0$ the surface $\Gamma^{\lambda}$ is a sphere, so that $D^{0}$ represents a shrinking sphere. For $\lambda$ close to $0, \Gamma^{\lambda}$ will be convex, and Huisken's theorem ensures that $D^{\lambda}(t)$ is a convex domain which shrinks to a point, and which becomes asymptotically spherical.

The surface $\Gamma_{\lambda}$ with $\lambda=1$ is not smooth: it consists of two tear shaped parts, and it has an isolated singularity at the origin. The domain $D^{\lambda}(0)$ with $\lambda=1$ has two components, as do all later domains $D^{1}(t)$ in the evolution $D^{1}$.

Each of the surfaces $\Gamma^{\lambda}$ is smooth and has at most one neck, for $\lambda<1$, so that the boundary of the inner evolution will be smooth for some time. Let $T_{\lambda}$ denote the first time at which $\partial D(t)$ is not a smooth hypersurface.

\subsection{Theorem.}

(i.) Assume that $n \geq 2$. There is a $\sigma \in(0,1)$ such that the solution to the mean curvature flow $\Gamma^{\sigma}(t)$ with initial data $\Gamma^{\sigma}(0)$ remains a smooth hypersurface with one neck until it shrinks to a point at time $t=T_{\sigma}$. In particular, $\Gamma^{\sigma}(t)$ remains nonconvex for $t<T_{\sigma}$.

(ii.) Assume that $n=1$. Then the inner evolution $D^{1}$ is not regular.

Proof. Denote the set $\lambda \in[0,1]$ for which $\Gamma^{\lambda}(t)$ has no necks for some time $t<T_{\lambda}$ by $J_{1}$. Denote the set of $\lambda \in[0,1]$ for which $\Gamma^{\lambda}(t)$ has two components for some $t>0$ by $J_{2}$. Note that $0 \in J_{1}$ and $1 \in J_{1}$.

(i) Lemma 5.5, on the evolution of hypersurfaces without necks, implies that $J_{1}$ and $J_{2}$ are disjoint. That is, once an inner evolution has no necks, it can no longer split into two parts.

If $\Gamma^{\lambda}(t)$ loses its only neck, then it follows from the continuous dependence of the classical solution (and its curvatures) on the parameter $\lambda$ that $J_{1}$ is open in $[0,1]$.

If $D^{\lambda}(t)$ has two components for some $t>0, \bar{D}^{\lambda}(t)$ also has two components for some $t>T_{\lambda}$. This follows from the moving cap lemma and the instantaneous smoothing of the solutions. We have observed that the evolution $D^{\lambda}(t)$ is regular for $0 \leq \lambda<1$. The regularity lemma 7.5 also implies that $D^{1}$ is regular since $\Gamma^{1}(0)$ has a singularity of the form $r=x^{2}$ at the origin. Thus, $\bar{D}^{\lambda}$ is the outer evolution for all $\lambda \in[0,1]$. Since $D^{\lambda}(0)$ increases as $\lambda$ decreases, the monotone convergence theorem now implies that $D^{\mu}(t)$ also has two components for $\mu$ close to $\lambda$. In other words, $J_{2}$ is open.

Since $J_{1}$ and $J_{2}$ are disjoint, nonempty sets, the set

$$
\Sigma=[0,1] \backslash\left(\left(J_{1} \cup J_{2}\right) \subset(0,1)\right.
$$

is nonempty. For $\sigma \in \Sigma$, we observe that $D^{\sigma}(t)$ always has a neck for $t<T_{\sigma}$. 


\section{Altschuler, Angenent and Giga}

It remains to prove that $\Gamma^{\sigma}(t)$ shrinks to a point at $t=T_{\sigma}$. By symmetry with respect to the $x=0$ hyperplane the neck must tend to the origin as $t \uparrow T_{\sigma}$. If $D^{\sigma}\left(T_{\sigma}\right)$ were nonempty, symmetry and the single point pinching argument would imply that $D^{\sigma}\left(T_{\sigma}\right)$ had two components. This would contradict $\sigma \notin J_{2}$. so $D^{\sigma}\left(T_{\sigma}\right)$ must be empty. As shown in $\S 5$, we now conclude that $\Gamma^{\sigma}(t)$ shrinks to a point at $t=T_{\sigma}$. This completes the proof of (i).

(ii) Throughout the paper, we have assumed that $n \geq 2$. However, the reader may wish to prove for himself that the monotone convergence theorem does not need this assumption. The work of Grayson, Gage and Hamilton [Gr1][GH] implies that $J_{1}=[0,1)$. If $D^{1}$ were regular, the monotone convergence theorem would imply that $D^{\mu}(t)$ also had two components for $\mu$ close to 1 . This would contradict $J_{1}=\left[0,1\right.$ ), so $D^{1}$ cannot be a regular evolution.

Q. E. D.

8.2. Theorem. Assume that $n \geq 2$. Let $\Gamma^{\sigma}(t)$ be as in the above theorem. If

$$
M(t)=\sup \left\{|\mathrm{A}(P, t)|: P \in \Gamma^{\sigma}(t)\right\}
$$

then

$$
\lim _{t \uparrow T_{\sigma}} \sup M(t) \sqrt{T_{\sigma}-t}=\infty
$$

Proof. Since $\Gamma^{\circ}(t)$ shrinks to a point, the attracting axis theorem implies that the mean curvature $H$ of $\Gamma^{\sigma}(t)$ is always nonnegative for $t$ close to $T_{\sigma}$. If (8.2) were false, then $\Gamma^{\sigma}(t)$ would be contained in a ball of radius $C\left(T_{\sigma}-t\right)^{1 / 2}$. Thus, the Huisken blow-up limit would be compact and have nonnegative mean curvature. By Huisken's classification of the limit, it would have to be a sphere. However, this would mean that $\Gamma^{\sigma}(t)$ became strictly convex at some time close to $T_{\sigma}$ which is a contradiction.

Q. E. D.

\subsection{Remark.}

(1) Theorem 8.1 (ii) provides a new example of nonregular motion with a rigorous proof. In [ES1] there is an example of the "figure-8" given without a proof.

(2) This theorem shows that there do exist surfaces which undergo "type II" blow up. Nevertheless the radius of the neck of such a solution tends to zero like $\left(T_{\lambda_{\bullet}}-t\right)^{1 / 2}$.

We next construct an asymmetric barbell which remains connected after it experiences a singularity. Let $\Gamma^{\lambda}(0)$ be defined as in (8.1). Assume that $n \geq 2$. As observed before, $\Gamma^{\lambda}(t)$ consists of two component for times $t>T_{\lambda}$ provided that $t$ is sufficiently close to $T_{\lambda}$ and that $\lambda$ is close to 1 . Since $T_{1}=0$, the monotone convergence theorem implies that $T_{\lambda} \downarrow 0$ as $\lambda \uparrow 1$. Let $B$ be a ball in $D^{1}(0) \cap\{x<0\}$ and let $T>0$ be the extinction time of $B$. Now, take $\lambda_{0}<1$ close enough to 1 so that $0<T_{\lambda_{0}}<T$ and that $\Gamma^{\lambda_{0}}(t)$ consists of two seperate pieces when $t>T_{\lambda_{0}}$ is sufficiently close to $T_{\lambda_{0}}$. 
Mean Curvature Flow Through Singularities for Surfaces of Rotation.

Consider the one-parameter family of inner evolutions $D_{\mu} \subset[0, \infty) \times \mathrm{R}^{n+1}$ whose initial domain $D_{\mu}(0)$ is the inside of the $C^{3}$ hypersurface $\Gamma_{\mu}(0) \subset \mathrm{R}^{n+1}$ where $\Gamma_{\mu}(0)$ is defined by the equation

$$
r^{2}=\left\{\begin{array}{l}
\left(1-\lambda_{0}+\lambda_{0} \mu x^{2}\right)^{2}\left(1-x^{2}\right) \text { for } x>0 \\
\left(1-\lambda_{0}+\lambda_{0} x^{2}\right)^{2}\left(1-x^{2}\right) \text { for } x \leq 0
\end{array}\right.
$$

Note that the surface $\Gamma_{1}(0)$ agrees with $\Gamma^{\lambda_{0}}(0)$ and $\Gamma_{0}(0)$ has no neck.

8.4. Theorem. Assume that $n \geq 2$. Then $\exists \nu \in(0,1)$ such that the generalized solution $\Gamma_{\nu}(t)$ with initial data $\Gamma_{\nu}(0)$ experiences only one singularity at time $t=t_{1}$ but remains connected until it shrinks to a point at $t=t_{\star}>t_{1} . \Gamma_{\nu}(t)$ is homeomorphic to a sphere for $0 \leq t<t_{\star}$.

Proof. We again appeal to a topological argument. Denote by $J_{1}$ the set $\mu \in[0,1]$ for which $D_{\mu}(t)$ has no necks for some time $t$ before it becomes singular at $t=t_{\mu}$. Denote by $J_{2}$ the set $\mu \in[0,1]$ for which $D_{\mu}(t)$ has two components for some $t>0$. As before, the complement $\Sigma$ of $J_{1} \cup J_{2}$ is a nonempty set in $(0,1)$.

Since $D_{\mu}(0) \supset B$, the set $D_{\mu}(t)$ is not empty for $t \leq T$. Since $0<t_{\mu} \leq T_{\lambda_{0}}<T$, the evolution $\Gamma_{\nu}$ for $\nu \in \Sigma$ becomes singular at time $t=t_{\nu}<T$ but remains connected until it disappears at time $t_{*}>T$. Since $\Gamma_{\nu}(0)$ has only one neck, $\Gamma_{\nu}(t)$ becomes singular only at time $t=t_{\nu}$ and $\Gamma_{\nu}\left(t_{\nu}\right)$ has its only singularity on the $x$-axis. The singularity is located at the right cap of $\Gamma_{\nu}\left(t_{\nu}\right)$ since $D_{\nu}\left(t_{\nu}\right)$ is connected. Thus, $\Gamma_{\nu}\left(t_{\nu}\right)$ is homeomorphic to a sphere for $0 \leq t<t_{\star}$. Since $\Gamma_{\nu}(t)$ has no necks for $t>t_{\star}$, it shrinks to a point at $t=t_{\star}$.

Q.E. D. 


$$
\text { Altschuler, Angenent and Giga }
$$

\section{References.}

[Alt] S. J. AlTSCHULER, Singularities of the curve shrinking flow for space curves, Journal of Differential Geometry Vol. 34, No. 2 (1991) 491-514.

[AGG] S. J. Altschuler, S. B. Angenent, AND Y. Giga, Generalized motion by mean curvature for surfaces of rotation, Advanced Studies in Pure Mathematics, to appear; also Hokkaido University Preprint \#118 (1991).

[AG] S. J. Altschuler AND M. GRAYSON, Shortening space curves and flow through singularities, Journal of Differential Geometry (1991) in press.

[A1] S. B. ANGENENT, The zeroset a a solution of a parabolic equation, Journal für die reine und angewandte Mathematik, 390 (1988), 79-96..

[A2] - , On the formation of singularities in the curve shortening problem, Journal of Differential Geometry, 33 (1991) 601-633.

[A3] - - Parabolic equations for curves on surfaces-part 2, Annals of Mathematics 133 (1991) 171-215.

[A4] - - Solutions of the 1-D porous medium equation are determined by their free boundary, Journal of the London Maths. Soc. 42 (1990) 339-353.

[A5] - - Shrinking Doughnuts, to appear in the proceedings of the conference on nonlinear parabolic PDE, Gregynog-Wales, august 1989.

[B] K. A. BRAKKE, The motion of a surface by its mean curvature, Math. Notes, Princeton, New Jersey, Princeton University Press (1978).

[XYC] X.-Y. CHEN, in preparation, .

[CM] X.-Y. CHEN AND H. MATANO, Convergence, asymptotic periodicity, and finite point blow-up in one dimensional semilinear heat equations, Journal of Differential Equations 78 (1989) 160-190.

[CGG1] Y.-G. CHEN, Y. GIGA AND S. GoTo, Uniqueness and existence of viscosity solutions of generalized mean curvature flow equations, Journal of Differential Geometry 33 (1991) 749-786; announcement in: Proc. Japan Acad. Ser. A 65 (1989) 207-210.

[CGG2] Y.-G. Chen, Y. GIGA AND S. Goto, Analysis toward snow crystal growth, to appear in: Proceedings of Functional Analysis and related topics, Sapporo 1990 (ed. S. Koshi); also Hokkaido University Preprint \#109, March 1991.

[CIL] M. G. CRANDALl, H. IsHII AND P. L. LIONS, User's guide to viscosity solutions of second order partial differential equations, preprint.

[DG] E. DEGIORGI, Some conjectures on flow by mean curvature, preprint.

[DK] G. DzIUK AND B. KAWOHL, On rotationally symmetric mean curvature flow, J. of Diff. Equations 93 (1991) 142-149.

[EH1] K. ECKER AND G. HUISKEN, Mean curvature for entire graphs, Annals of Mathematics 130 (1989) 453-471.

[EH2] K. ECKER AND G. HUISKEN, Interior estimates for hypersurfaces moving by mean curvature, Inventiones Mathematicae 105 (1991) 547-569.

[ESS] L. C. Evans, H. M. Soner and P. E. Souganidis, Phase Transitions and generalized mean curvature flow equations, to appear in Comm. Pure Appl. Math.. 
Mean Curvature Flow Through Singularities for Surfaces of Rotation.

[ES1] L. C. Evans AND J. SPRUCK, Motion of level sets by mean curvature I, Journal of Differential Geometry 33 (1991) 635-681.

[ES2] - - Motion of level sets by mean curvature II, to appear in: Trans. Amer. Math. Soc..

[ES3] - - Motion of level sets by mean curvature III, preprint.

[GH] M. GAGE AND R. S. HAMILTON, The heat equation shrinking convex plane curves, Journal of Differential Geometry 23 (1986) 69-96.

[GG1] Y. GIGA AND S. GoTo, Motion of hypersurfaces and geometric equations, to appear in J. Math. Soc. Japan 44 (1992).

[GG2] - - Geometric evolutions of phase boundaries, IMA preprint \#738.

[GGI] Y. GIGA, S. GoTo AND H. IsHII, Global existence of weak solutions for interface equations coupled with diffusion equations, to appear in SIAM J. Math. Annal..

[GGIS] Y. GigA, S. Goto, H. IshII AND M.-H. SATo, Comparison principle and convexity preserving properties for singular degenerate parabolic equations on unbounded domains, Indiana University Math. Journal 40 (1990) 443-470.

[GK] Y. GIGA AND R. KoHN, Asymptotically self-similar blow-up of semilinear heat equations, Comm. Pure and Appl. Math. 38 (1985) 297-319.

[GP] Victor A. Galaktionov and Sergei A. Posashkov, On some monotonicity in time properties for a quasilinear parabolic equation with source, to appear in the proceedings of the conference on Degenerate Diffusion Equations, IMA, May 12-17, 1991.

[GrI] M. GRAYSON, The heat equation shrinks embedded plane curves to round points, Journal of Differential Geometry 26 (1987) 285-314.

[Gr2] - - A short note on the evolution of a surface by its mean curvature, Duke Math. Journal 58 (1989) 555-558.

[H1] G. HUISKEN, Flow by mean curvature of convex surfaces into spheres, Journal of Differential Geometry 20 (1984) 237-266.

[H2] - - Asymptotic behaviour for singularities of the mean curvature flow, Journal of Differential Geometry 31 (1991) 285-299.

[H3] - - Local and Global Behaviour of hypersurfaces moving by mean curvature, C. M. A. Australian National University Preprint CMA-R34-90 (1990).

[I1] T. ILMANEN, Generalized flow of sets by mean curvature on a manifold, preprint.

[I2] - - The level-set flow on a manifold, preprint.

[Ko] N. KoREVAAR, An easy proof of the interior gradient bound for solutions to the prescribed mean curvature equation, Nonlinear functional analysis and its applications, Proc. Symp. Pure Math. 45 (1986) part 2, 81-89.

[LUS] O. A. LADYZHENSKaya, V. SOlonNIKov AND N. URAL'CeVA, Linear and quasilinear equations of parabolic type, Translations of Mathematical Monographs, vol. $23 \mathrm{~A}$. M. S. (1968).

[Ma] H. MATANo, Nonincrease of the lapnumber for a solution of a one-dimensional semilinear parabolic equation, J. Fac. Sci. Univ. Tokyo, Sec.IA, 29 (1982) 401-441. 


\section{Altschuler, Angenent and Giga}

[OJK] T. OHTA, D. JASNOW AND K. KAWASAKI, Universal scaling in the motion of random interfaces, Physics Review Letters 49 (1982) 1223-1226.

[OS] S. Osher AND J. A. SETHIAN, Fronts propagating with curvature dependent speed: Algorithms based on Hamilton-Jacobi formulations, J. Comp. Phys. 79 (1988) 12-49.

[Se1] J. A. Sethian, Curvature and evolution of fronts, Comm. Math. Physics 101 (1985) $12-49$.

[Se2] - - , Numerical for propagating interfaces: Hamilton-Jacobi equations and conservation laws, Journal of Differential Geometry 31 (1990) 131-161.

[So] H. M. SONER, Motion of a set by the curvature of its boundary, to appear in: Journal of Differential Equations.

[SS] H. M. SONER AND P. E. SoUgANIDIs, Uniqueness and singularities of cylindrically symmetric surfaces moving by mean curvature, preprint.

Steven J. Altschuler

Centre for Mathematics and its Applications

Australian National University

GPO Box 4

Canberra, ACT-2601 Australia

Sigurd B. Angenent

Department of Mathematics, Van Vleck Hall

University of Wisconsin, Madison

Madison, Wisconsin 53706 USA

Yoshikazu Giga

Department of Mathematics

Hokkaido University

Sapporo 060, Japan 

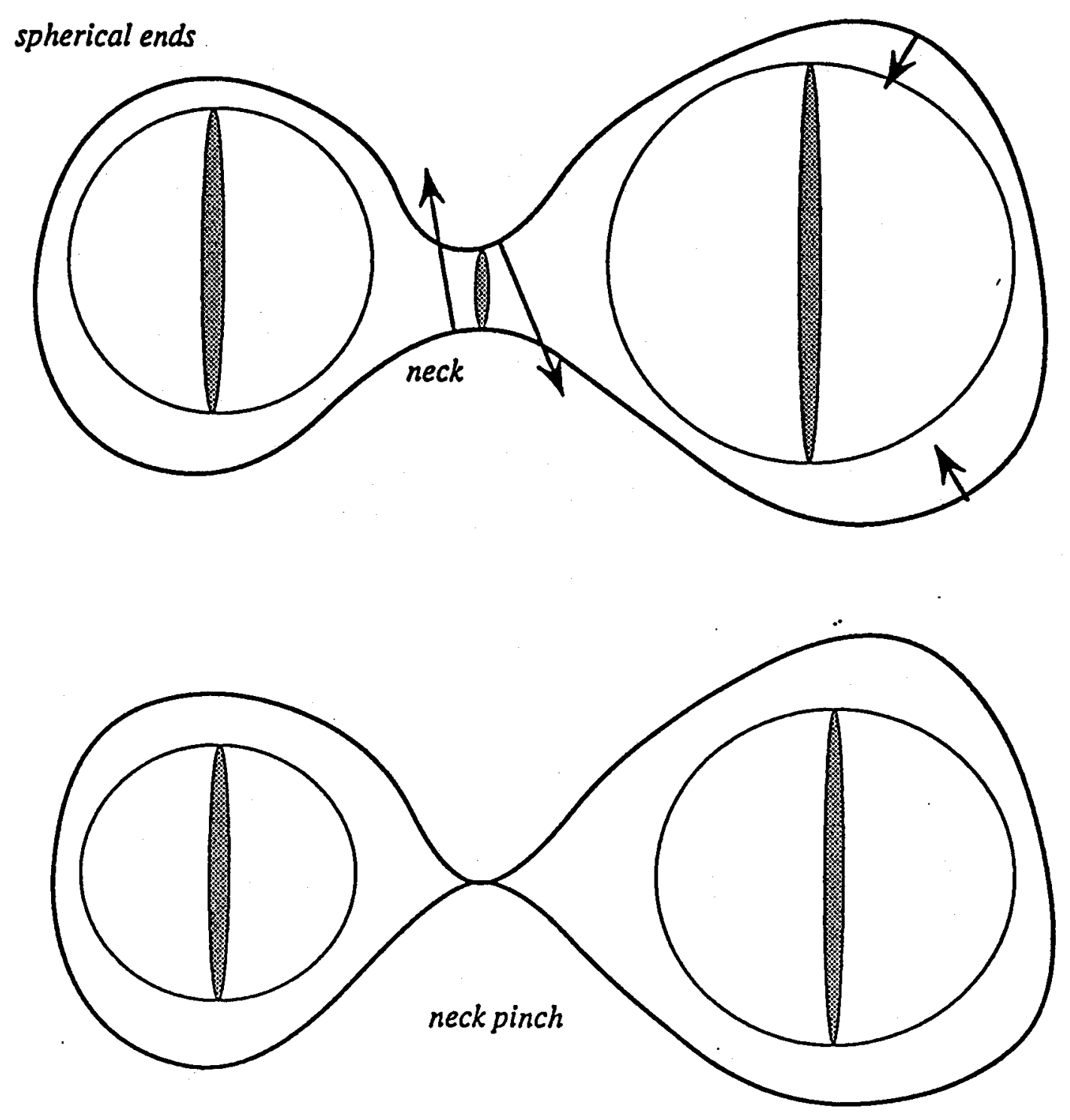

figure 1.1 


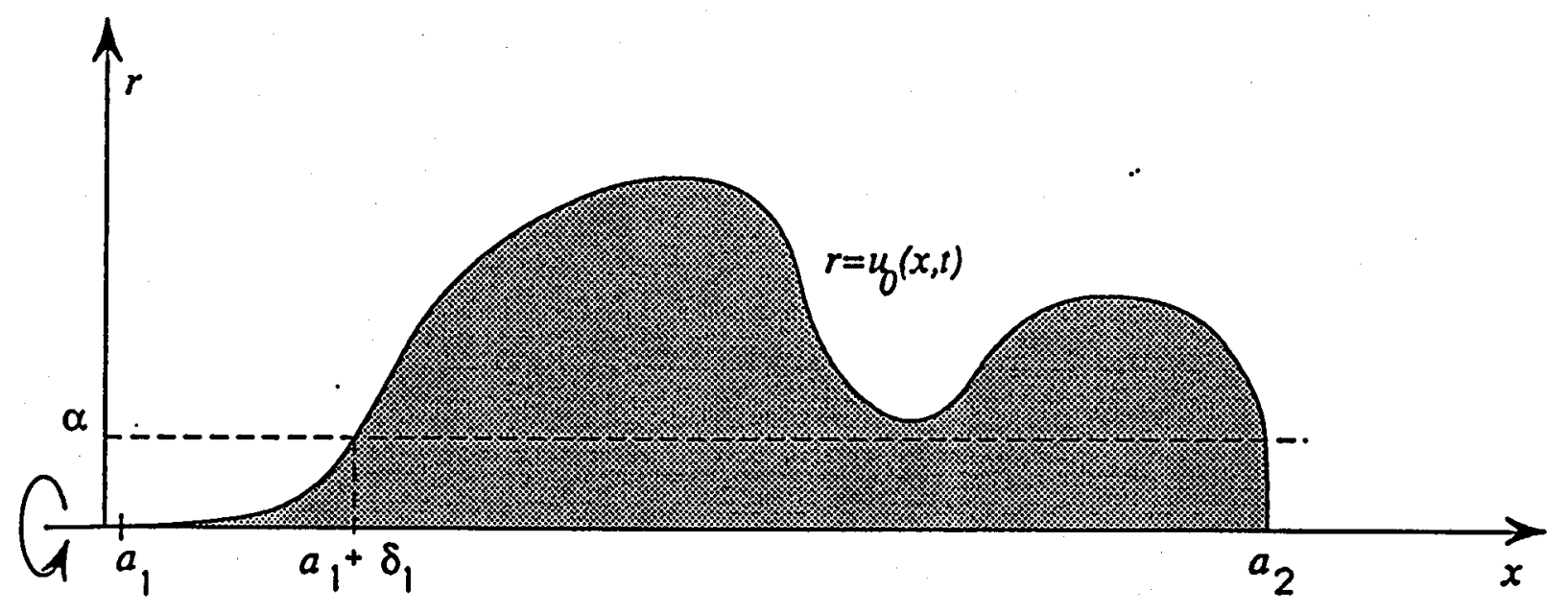

figure 2.1 


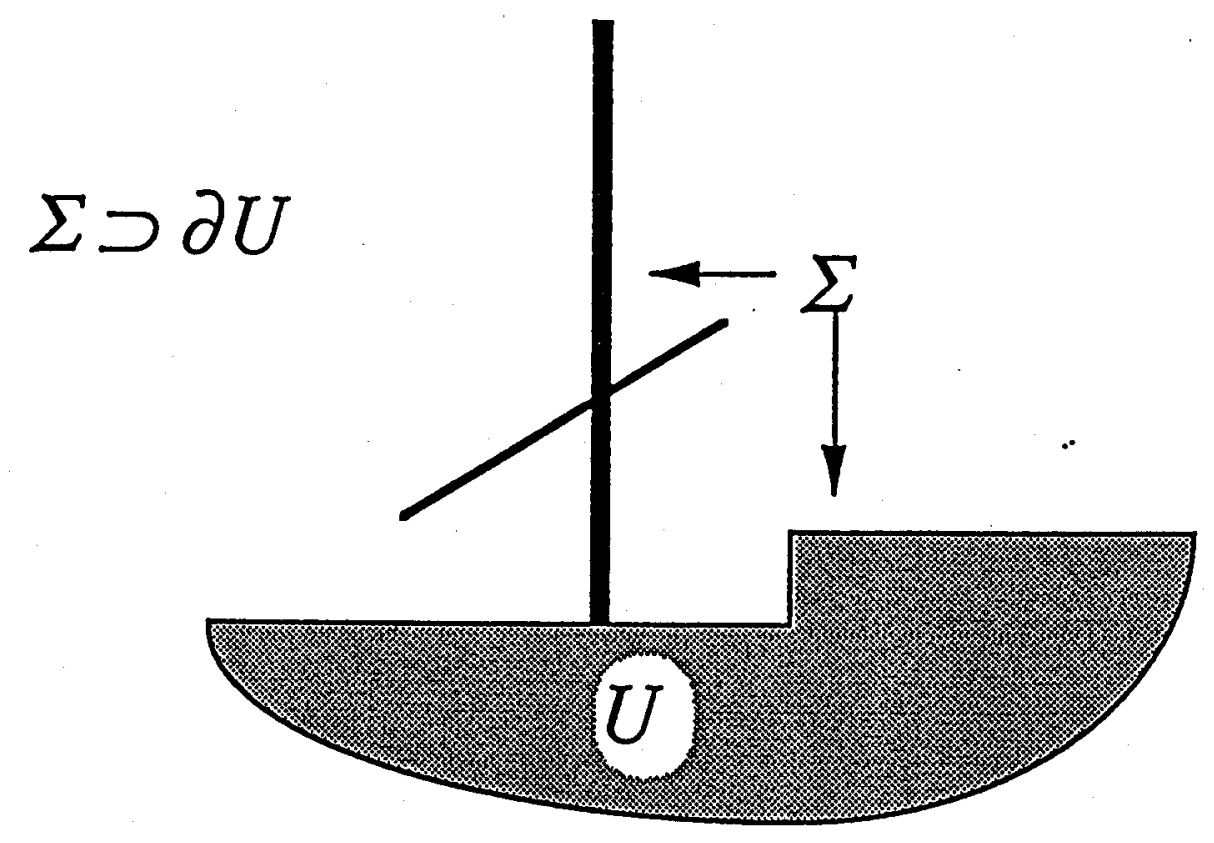

figure 3.1 


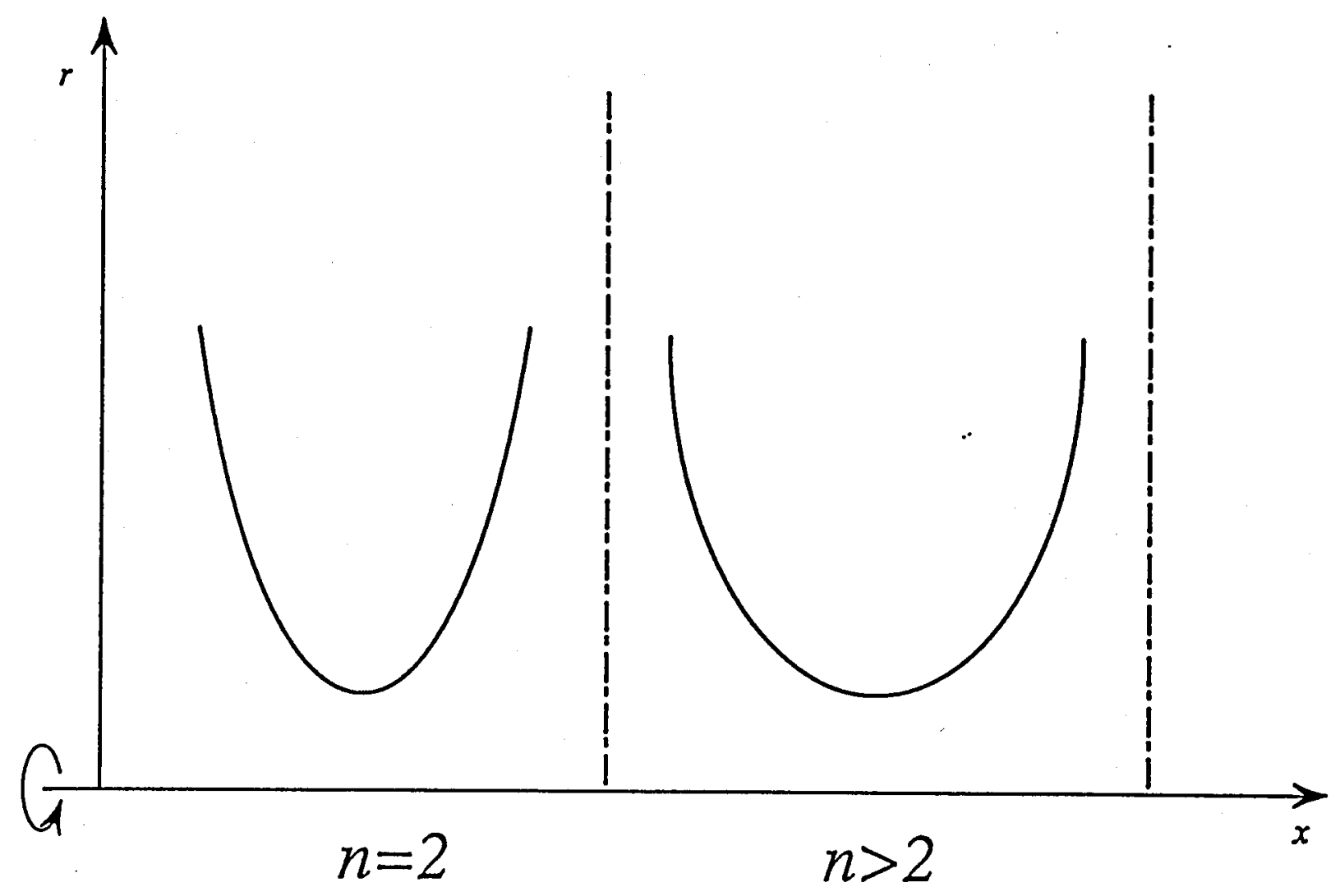

figure 4.1 

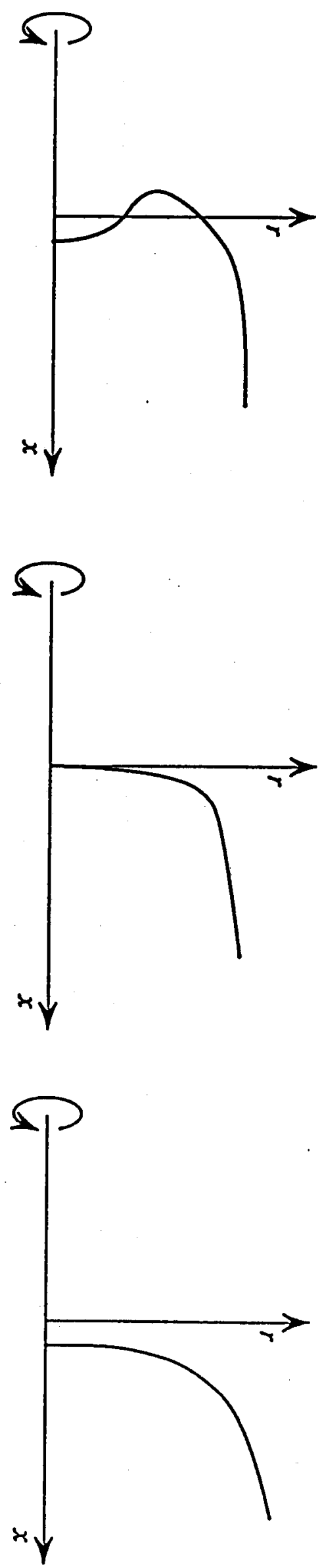

figure 4.2 
tet 


$$
\text { tew }
$$




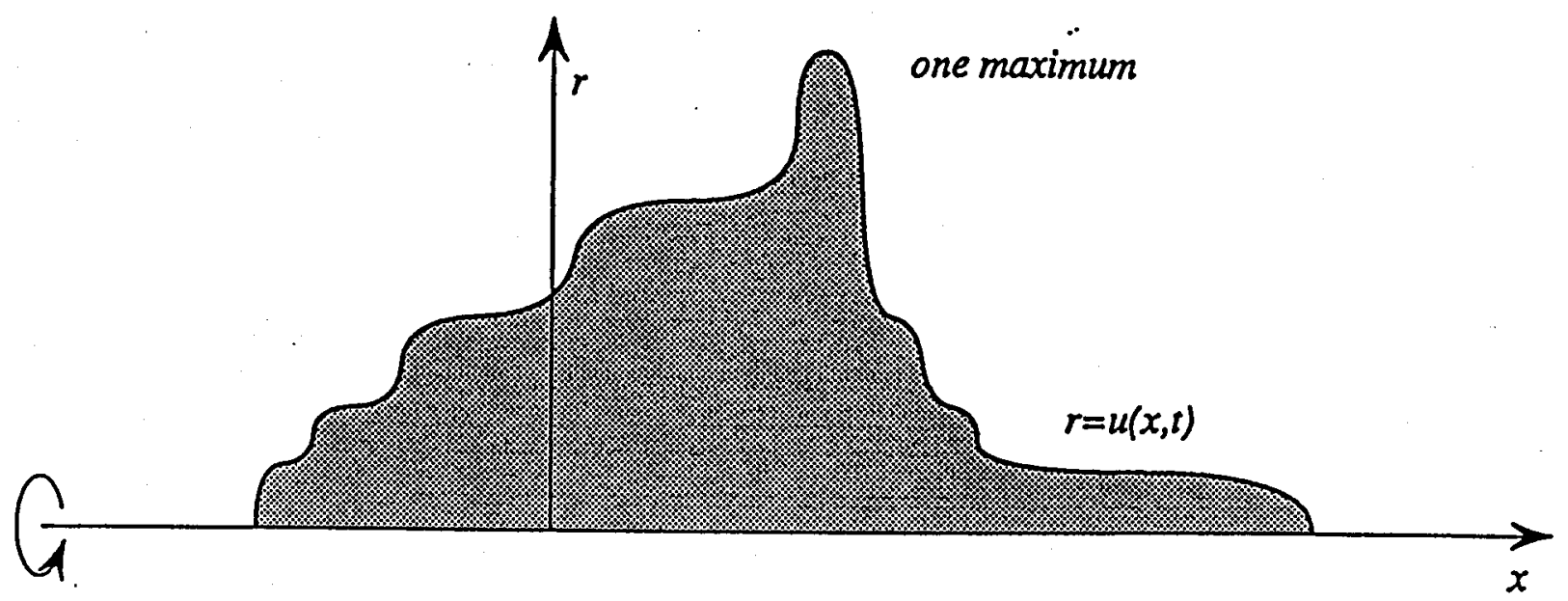

figure 5.1 


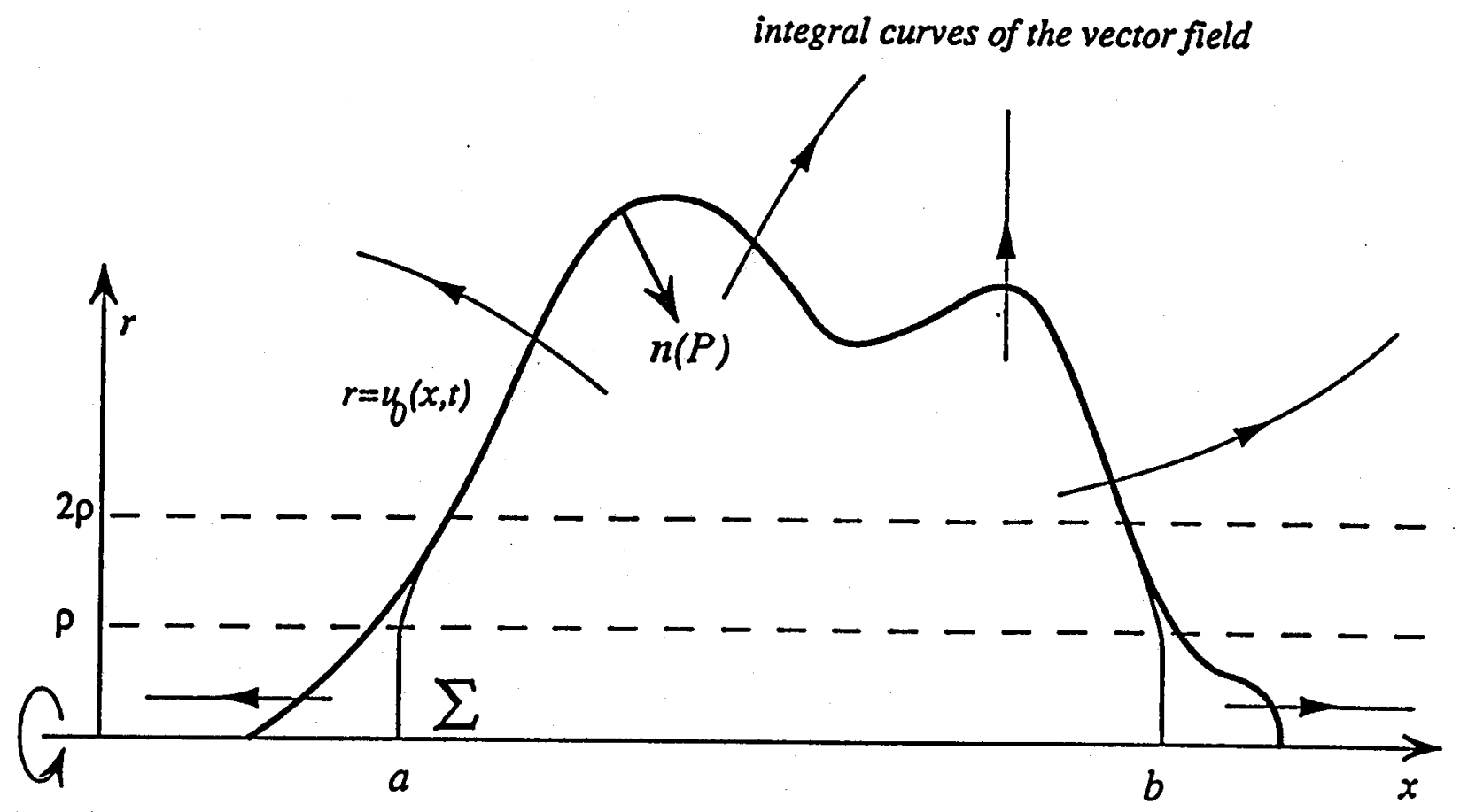

figure 7.1 